\title{
Hybrid Organic on Inorganic Semiconductor Heterojunction
}

\author{
by \\ Chih-Hsien Chen \\ Department of Electrical and Computer Engineering \\ McGill University \\ Montreal, Canada
}

February, 2006

A thesis submitted to the Faculty of Graduate Studies and Research in partial fulfillment of the requirements for the degree of Master of Engineering

(C) Chih-Hsien Chen 2006 


$\begin{array}{ll}\begin{array}{l}\text { Library and } \\ \text { Archives Canada }\end{array} & \begin{array}{l}\text { Bibliothèque et } \\ \text { Archives Canada }\end{array} \\ \begin{array}{l}\text { Published Heritage } \\ \text { Branch }\end{array} & \begin{array}{l}\text { Direction du } \\ \text { Patrimoine de l'édition }\end{array} \\ \begin{array}{l}\text { 395 Wellington Street } \\ \text { Ottawa ON K1A 0N4 }\end{array} & \begin{array}{l}\text { 395, rue Wellington } \\ \text { Ottawa ON K1A ON4 } \\ \text { Canada }\end{array} \\ \end{array}$

Your file Votre référence ISBN: 978-0-494-24946-8 Ourfile Notre référence ISBN: 978-0-494-24946-8

NOTICE:

The author has granted a nonexclusive license allowing Library and Archives Canada to reproduce, publish, archive, preserve, conserve, communicate to the public by telecommunication or on the Internet, loan, distribute and sell theses worldwide, for commercial or noncommercial purposes, in microform, paper, electronic and/or any other formats.

The author retains copyright ownership and moral rights in this thesis. Neither the thesis nor substantial extracts from it may be printed or otherwise reproduced without the author's permission.
AVIS:

L'auteur a accordé une licence non exclusive permettant à la Bibliothèque et Archives Canada de reproduire, publier, archiver, sauvegarder, conserver, transmettre au public par télécommunication ou par l'Internet, prêter, distribuer et vendre des thèses partout dans le monde, à des fins commerciales ou autres, sur support microforme, papier, électronique et/ou autres formats.

L'auteur conserve la propriété du droit d'auteur et des droits moraux qui protège cette thèse. $\mathrm{Ni}$ la thèse ni des extraits substantiels de celle-ci ne doivent être imprimés ou autrement reproduits sans son autorisation.
In compliance with the Canadian

Privacy Act some supporting forms may have been removed from this thesis.

While these forms may be included in the document page count, their removal does not represent any loss of content from the thesis.
Conformément à la loi canadienne sur la protection de la vie privée, quelques formulaires secondaires ont été enlevés de cette thèse.

Bien que ces formulaires aient inclus dans la pagination, il n'y aura aucun contenu manquant. 


\section{Abstract}

Hybrid organic on inorganic semiconductor heterojunctions with a sandwich structure have been fabricated and studied using conjugated polymers. The inorganic semiconductor was n-type silicon substrate. The conjugated polymers used in this work include poly(2-methoxy-5-(2-ethylhexyloxy)-1,4-phenylenevinylene) containing polyhedral oligomeric silsesquioxanes (MEH-PPV POSS), regioregular poly(3-hexylthiophene) (RR-P3HT) and poly(3,4-ethylenedioxythiophene) (PEDOT).

Current-voltage (I-V) and capacitance-voltage (C-V) measurements were performed to characterize the devices. All the devices displayed a rectifying characteristic. Among these devices, the first ever reported PEDOT doped with $\mathrm{BF}_{3}$ on $\mathrm{n}$-Si heterojunction devices showed the best performance with a rectification ratio around $5.7 \times 10^{5}$ at $\pm 2 \mathrm{~V}$ and an ideality factor of 2.3. It is speculated that performance of the devices is inversely proportional to the potential barrier height at the organic-inorganic interface. Other results from this work also suggested that smaller energy level offset between the HOMO of the conjugated polymer and the work-function of anode metal will improve device performance. Contact resistance between the anode and polymer is large $(\sim \mathrm{M} \Omega$ range) and should be further investigated in the future. 


\section{Résumé}

Des hétérojonctions ont été fabriquées par le dépôt des couches de matériaux organiques et hybrides sur des semi-conducteurs inorganiques et ces structures ont été étudiées par des polymères conjugués. Le semi-conducteur inorganique était du silicium de type n. Les polymères conjugués utilisés dans ce rapport sont poly(2-méthoxy-5(2-ethylhexyloxy)-1,4-phenylenevinylene) qui contiennent des silsesquioxanes oligomères polyhedral (MEH-PPV POSS), poly(3-hexylthiophene) regioregular (RR-P3HT) et poly(3,4-ethylenedioxythiophene) (PEDOT).

Des mesures de courant-tension (I-V) et de la capacité-tension $(\mathrm{C}-\mathrm{V})$ ont été effectuées pour caractériser les dispositifs. Tous les dispositifs ont montré une caractéristique I-V non symétrique où les rectifications pouvaient être observées. Parmi ces dispositifs, le PEDOT dopé de $\mathrm{BF}_{3}$ a été déposé sur du n-Silicium et cette hétérojonction a démontré la meilleure performance avec un rapport de rectification autour de $5.7 \times 10^{5}$ au $\pm 2 \mathrm{~V}$ et un facteur d'idéalité de 2.3. La spéculation suggére que la performance des dispositifs est inversement proportionnelle à la taille de la barrière de potentiel à l'interface organique-inorganique. D'autres résultats de ce travail démontrent aussi qu'avec un plus petit niveau d'énergie entre le HOMO du polymère conjugué et la travail de sortie du métal utilisé comme anode améliorerait la performance des hétérojonctions. La résistance de contact entre l'anode et le polymère est grande $(\sim \mathrm{M} \Omega)$ et devrait être plus étudiée à l'avenir. 


\section{Acknowledgements}

I wish to take this opportunity to gratefully acknowledge all those who gave me the possibility to complete this thesis.

Firstly, I would like to express my sincere gratitude to my supervisor, Dr. Shih for his guidance, endless patience and invaluable assistance during this work.

Secondly, I thank Dr. Steven Xiao of Organic Vision Inc. for providing some of the polymers for this work. I would like to thank the technicians from ECE Mechanical Workshop for allowing me to use their equipment and their help in creating some of the hardware needed in this work.

I would also like to thank my fellow colleagues in the Electronic Devices and Materials Lab for their stimulating discussions, countless help and general advices. Special thanks go to Hung-Pao Yang and Jeanne Shih for French translation of the thesis abstract.

Last and definitely not the least, I am forever grateful to my parents and brothers for their understanding, endless patience and encouragement when it was most required. 


\section{Contents}

ABSTRACT TÉSUÉ

3. ORGANIC ON INORGANIC SEMICONDUCTOR HETEROJUNCTION THEORY ...........20

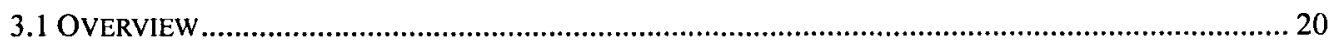

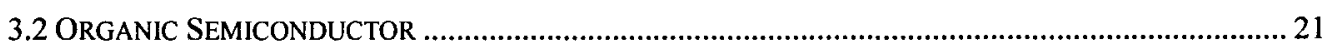

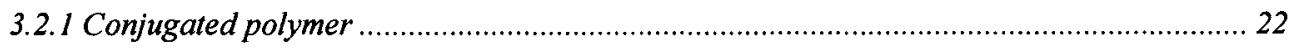

3.2.2 Electronic energy structure of conjugated polymer....................................................... 25

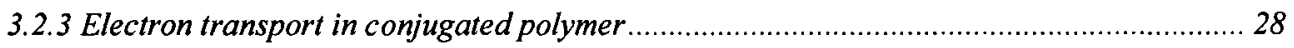

3.3 HETEROJUNCTION .............................................................................................................. 31

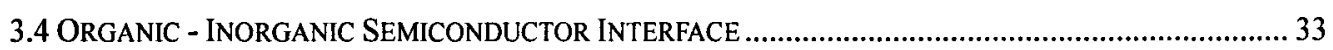

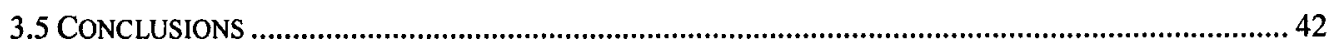

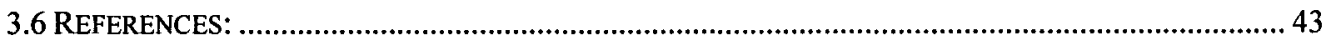

\section{FABRICATION OF ORGANIC ON INORGANIC SEMICONDUCTOR HETEROJUNCTION}

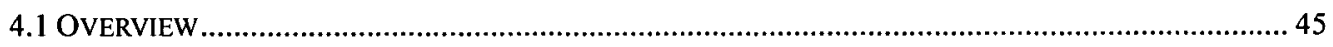

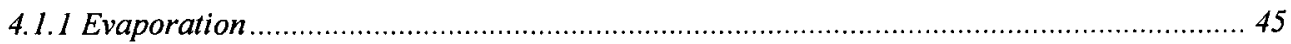

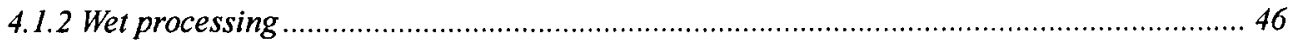

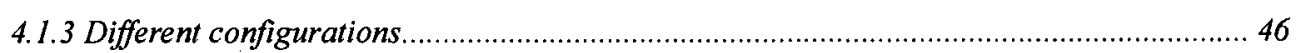

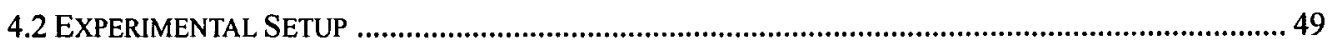

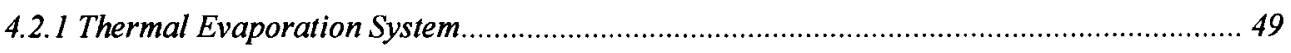

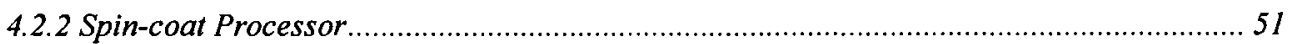




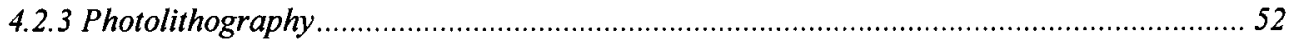

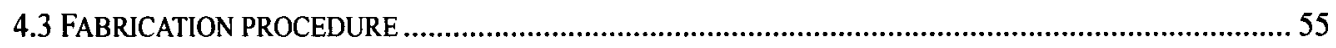

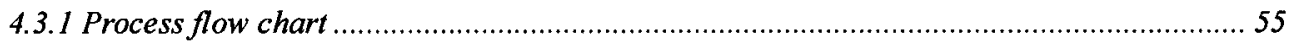

4.3.2 Description of different fabrication steps ................................................................. 58

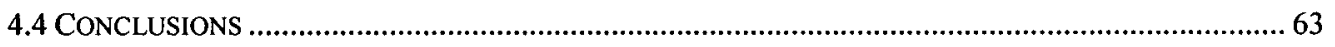

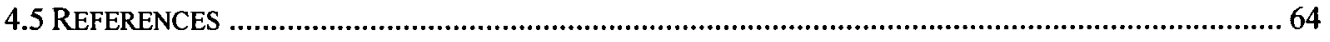

5. MEASUREMENT SETUP AND EXPERIMENTAL RESULTS ............................................65

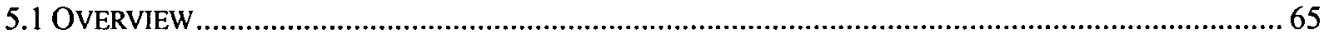

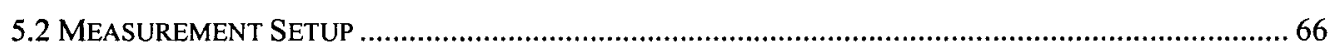

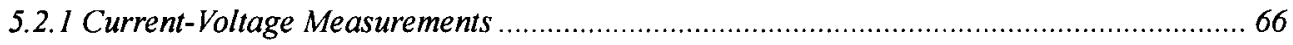

5.2.2 Capacitance-Voltage Measurements........................................................................ 71

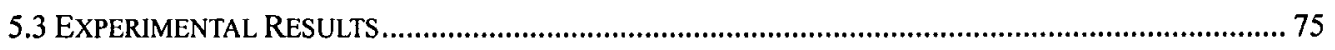

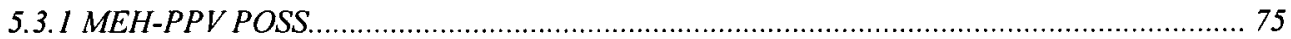

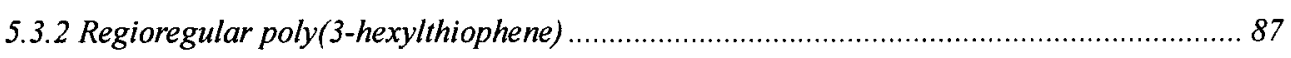

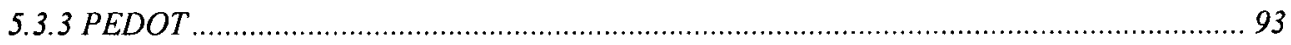

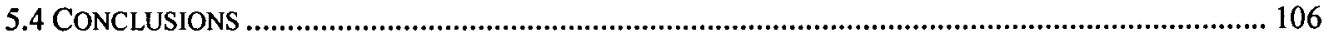

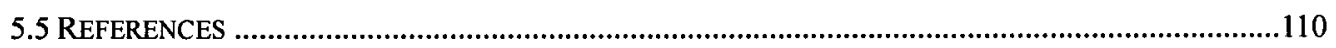

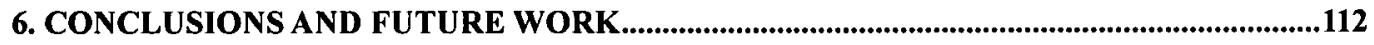




\section{Introduction}

Robert Noyce and Jack Kilby co-invented the integrated circuit in 1959. Since then, inorganic silicon semiconductors, silicon dioxide insulators, and metal such as aluminium and copper have been the backbone of the semiconductor industry. However in the last two decades, there has been a growing interest in organic electronics. Research efforts have been made to improve the optical and electrical properties of these organics materials and their associated devices such as organic light-emitting devices (OLED), organic field-effect transistors (OFET) and organic photovoltaic in an attempt to replace their inorganic counterparts.

Organic materials have the key advantages of simple and low-temperature thin film processing through inexpensive techniques such as spin coating. In addition, the flexibility of organic chemistry enables the formation of organic molecules with useful luminescent and conducting properties. However, the electrical properties of organic semiconductor devices often degrade when exposed to unfavourable environments. Moreover, organic semiconductors are often attacked by many of the chemicals that are used in conventional device processing. These two factors have limited the practical use of organic semiconductors despite their demonstrated application to optoelectronic devices. 
Nevertheless, considerable progress has been made in realizing practical active electronic and optoelectronic devices where an organic material forms an integral part of the device structure. One promising approach employs a thin organic film that is layered onto the surface of a conventional inorganic semiconductor substrate to form a hybrid organic on inorganic semiconductor heterojunction device. The main advantage of such hybrid devices is the possibility that the composition of the organic film can be altered only slightly to effect large changes in its optical and electronic properties. Furthermore, different combinations of organic and inorganic semiconductor can be utilized to obtain different desirable properties or applications.

Therefore, the purpose of this project is to investigate the characteristics of the hybrid organic on inorganic semiconductor heterojunction devices through techniques such as current-voltage and capacitance-voltage measurements. Organic polymers chosen for the fabrication of these organic on inorganic semiconductor heterojunctions include MEH-PPV POSS, RR-P3HT and PEDOT, while the inorganic counterpart is n-type silicon substrate. Metals used for the contact in this work include gold, aluminium and magnesium-silver alloy. Different metal work functions will have different effects on the characteristics of the devices due to the metal-organic interfaces at the contact. Results from these hybrid heterojunctions hopefully will help in understanding the operation of organic on inorganic semiconductor heterojunction 
devices.

The next chapter will give a summary for some of the research literature related to the organic on inorganic semiconductor devices over the last two decades. Chapter 3 will describe the theory of organic materials with emphasis on conjugated organic polymers. Possible transport mechanisms at the interface between organic and inorganic heterojunctions will be explained as well. Chapter 4 provides the detailed fabrication procedure for the hybrid organic on inorganic semiconductor heterojunction devices. Measurement setup and device characteristics for 3 different sets of heterojunctions are presented in Chapter 5. The concluding chapter, Chapter 6, summarizes this work including recommendations for future development. 


\section{Literature Survey \\ 2.1 Overview}

During the past decades, intensive research on semiconductor heterostructures

has led to the discoveries of many new physical phenomena and device concepts such as semiconductor lasers and photodetectors. A new class of heterostructures [2.1] consisting of contacts between organic and inorganic semiconductors has been reported. In particular, heterojunctions consisting of a thin layer of a molecular semiconductor deposited onto the surface of an inorganic semiconductor substrate such as $\mathrm{Si}, \mathrm{GaAs}$, or InP have been found to form rectifiers with characteristics similar to ideal p-n junctions [2.1]. The high index of refraction, high transmittance over broad spectral regions and the unique electronic properties of many organic semiconductors suggest uses for such hybrid structure in many optoelectronic device applications such as organic-on-inorganic semiconductor field-effect transistors [2.2].

Organic materials have the key advantages of simple and low-temperature thin film processing through inexpensive techniques such as spin coating, ink-jet printing, or stamping. In addition, the flexibility of organic chemistry enables the formation of organic molecules with useful luminescent and conducting properties. Over the last few years, the carrier mobilities of organic channel layers in organic field-effect transistors (OFETs) have increased dramatically from $10^{-4}$ to $1 \mathrm{~cm}^{2} / \mathrm{V}-\mathrm{s}$ (comparable 
to those of amorphous silicon) [2.3]. Many applications of these organic heterojunctions devices have been reported including photovoltaic [2.4] and electroluminescent $[2.5,2.6]$ devices. Ultra-thin layers of these organic materials and multiple quantum-well structures [2.7] have also been fabricated by depositing the organic semiconductors under ultra high vacuum conditions.

However, the electrical properties of organic-inorganic semiconductor heterojunctions can degrade when exposed to unfavourable environments. In addition, organic semiconductors are often attacked by many of the chemicals that are used in conventional device processing. These two factors have limited the practical use of organic semiconductor despite their demonstrated application to optoelectronic devices. Furthermore, organic compounds generally have a number of disadvantages, including poor thermal and mechanical stability. In addition, while electrical transport in organic materials has been improved, the room-temperature mobility is fundamentally limited by the weak van der Waals interactions between organic molecules (as opposed to the stronger covalent and ionic forces found in inorganic systems). The stability and electrical transport characteristics of organic materials lead to a reduced device lifetime. Other drawbacks of the organic materials include the difficulty to dope some of the organic semiconductors with trace impurities in order to alter their majority carrier type and also the difficulty in achieving good crystallinity, 
or long-range crystalline order in the organic material.

In spite of these difficulties, considerable progress has been made in realizing practical, active electronic and optoelectronic devices where an organic material forms an integral part of the device structure. One promising approach, as mentioned before, employs an organic film that is layered onto the surface of a conventional inorganic semiconductor substrate to form an insulating or conducting layer that controls the distribution of electric fields and hence the transport of charges within the device. An attractive feature of such devices is that the composition of the organic film can be altered only slightly to effect large change in its optical and electronic properties. Furthermore, the cohesive forces that bind molecules are due to relatively weak van der Waals dipolar attractions [2.8]. Hence, the materials are somewhat soft (as compared to $\mathrm{Si}$, for example), and can thus be layered without inducing strain onto a variety of semiconductor substrates if the organic material is grown in vacuum of small molecules. Thus, organic/inorganic semiconductor growth process need not be limited by the constraints of lattice-matching. Usually, the lattice-matching restriction limits the selection on the particular inorganic semiconductors that can be combined without inducing large strains, and hence lattice defects. However, no such constraints exist for organic-on-inorganic semiconductor material combinations. The organic on inorganic semiconductor heterojunctions appear to be an advantageous combination 
of many of the properties of both organic and inorganic materials.

In the past twenty years, many research groups have been working on the active organic on inorganic (OI) devices, while most of the investigations were on the characterization of different hybrid organic on inorganic devices such as photovoltaic, light-emitting devices or field-effect transistors. Some of the organic materials that have been studied include perylenetertracarboxylic dianhydride (PTCDA), poly (3-methylithiophene) (PMeT), polyethylene dioxythiophene (PEDOT), polypyrrole, pyronine-B, polyacetylene and so on. Common methods of preparation for the organic materials are electrodeposition, galvanostatic oxidation, thermal evaporation (sublimation) or spin-coating. On the other hand, some of the inorganic semiconductor materials that have been used include $\mathrm{Si}, \mathrm{CdSe}, \mathrm{CdS}, \mathrm{GaAs}, \mathrm{InP}$ and Ge. In the next following sections, a brief summary of the work performed by different research groups related to organic on inorganic semiconductor heterojunctions will be given with emphasis on the organic materials used, device performances and their potential applications. 


\subsection{PTCDA}

One of the earliest organic on inorganic semiconductor heterostructures reported was the one with large rectifying barrier proposed by S. F. Forrest, M.L. Kaplan and P. H. Schmidt in 1984. These rectifying barriers are formed between polycyclic aromatic molecular compound 3,4,9,10-peryleneteracarboxylic dianhydride (PTCDA) and Si substrates $[2.1,2.9]$. Refer to Figure 2.1 for a unit cell of PTCDA. It has been found that the reverse-biased current-voltage characteristics of the organic on inorganic diodes are similar to diffused inorganic p-n junctions. Furthermore, they have shown that the I-V characteristics can be understood under both forward and reverse bias by assuming that the current is limited by thermionic emission of charges over the OI contact barrier at low applied voltages, and by space-charge injection into the organic thin film at high current densities [2.9]. In the following year, they also reported that OI contact barriers can also be formed by deposition of PTCDA or its derivative compound, N, N' dimethyl 3,4,9,10-perylenetetracarboxylic dimmide (DIME_PTCDI) on InP-based alloys [2.10] as well as on GaAs [2.11]. In the case of InP and related compounds, the barrier heights are considerably smaller than those attained with Si. 


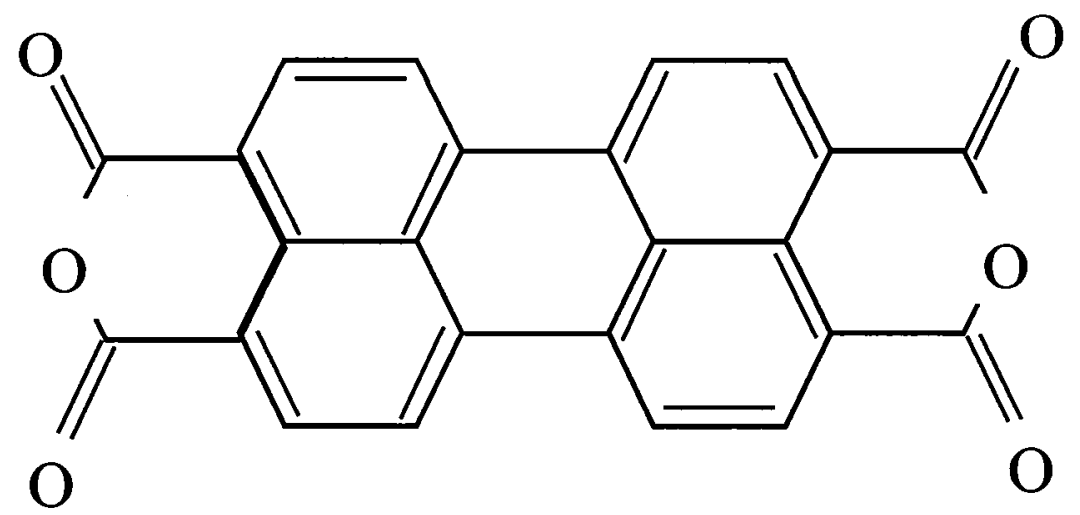

Figure 2.1: Chemical structure of a unit cell of PTCDA. [2.8] 


\subsection{PMeT}

Around the same period of time, G. Horowitz and F. Garnier have reported polythiophene-GaAs p-n heterojunctions solar cells $[2.12,2.13]$. Thin layers of poly (3-methylthiophene) (PMeT) polymer have been grown on n-type GaAs substrates to form a p-n junction. An electrochemical polymerization technique was used, which allowed the deposition of thin $(25 \mathrm{~nm})$ films. A gold overlayer was then evaporated to give an ohmic contact to the polymer. The reported heterojunctions differed from the already described organic-on-inorganic structures in that most of the incident light was absorbed within the GaAs substrate and not in the polymer front layer. Photocurrent spectra later on confirmed that the light was principally absorbed in the n-GaAs [2.14]. Figure 2.2 shows suggested energy band diagram of PMeT/GaAs device. In the literature, comparison of PMeT/GaAS heterojunctions and $\mathrm{Au} / \mathrm{GaAs}$ junction showed that the slight decrease of short-circuit current, due to the absorption of light by the polymer, was overcompensated by a $57 \%$ increase of the open-circuit voltage. The improvement of $\mathrm{V}_{O C}$ was attributed to the creation of a high built-in potential of $0.93 \mathrm{eV}$, together with the reduction of the dark direct current. The power characteristics of these cells showed an increase of energy conversion efficiency on passing from Schottky cell (14\%) to the hybrid cell (17.5\%) [2.14]. 


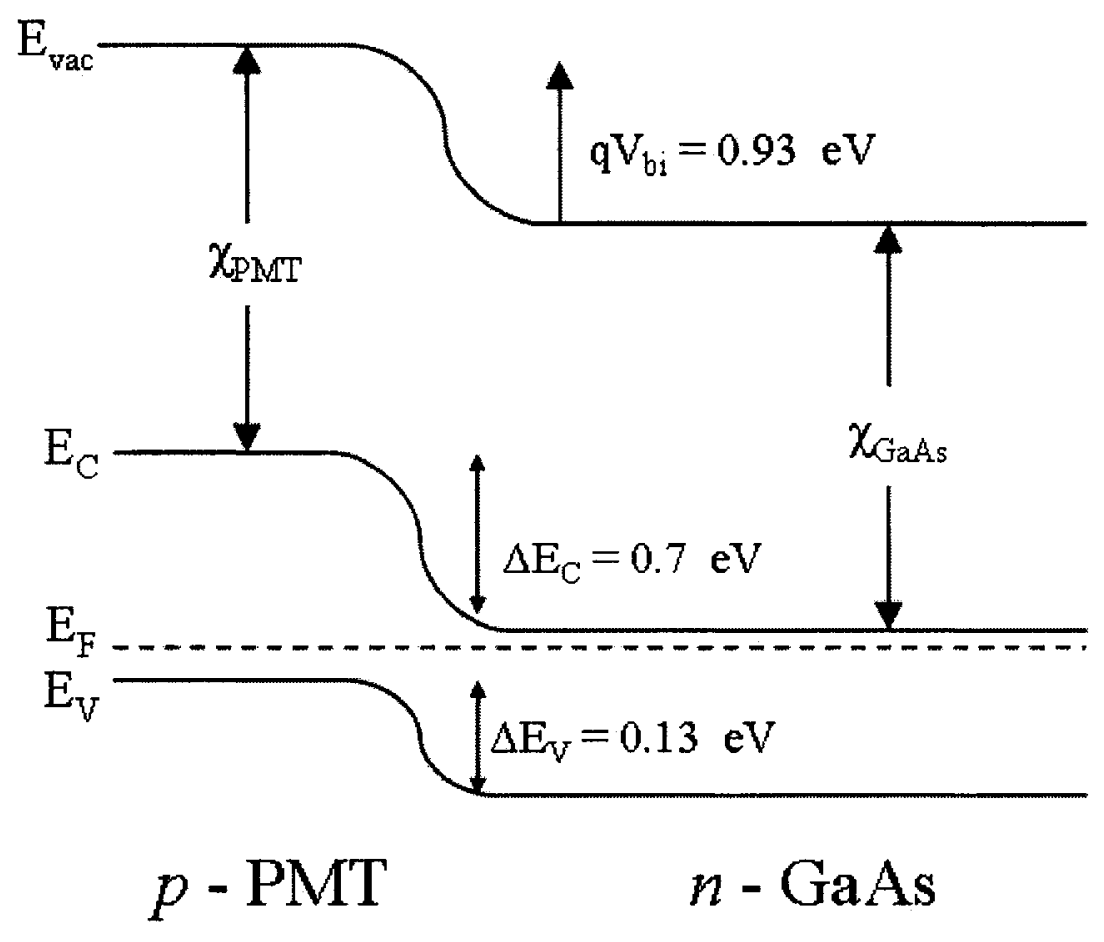

Figure 2.2: Energy band diagram of the hybrid n-GaAs/p-PMeT cell [2.14].

In 1990s, more publications of organic on inorganic semiconductor devices utilizing PMeT as the organic material were reported. One of the groups that have been working on PMeT was led by P. Chartier. Polycrystalline cadmium selenide (CdSe) layers were first deposited onto indium tin oxide (ITO) glass by chemical bath deposition. Electrodeposition of PMeT was then carried out on the CdSe surface. The hybrid organic-inorganic $\mathrm{PMeT} / \mathrm{CdSe}$ non-optimized junction exhibited a photovoltaic behaviour with energy conversion efficiency, under $56 \mathrm{mWcm}^{-2}$ white light from xenon lamp, of $1.3 \%$, uncorrected for the light losses by absorption when shined through Pyrex glass/ITO bulk materials [2.15]. Later on, hybrid 
organic-inorganic thin film photovoltaic junctions $\mathrm{PMeT}(\mathrm{Y}) / \mathrm{CdS}(\mathrm{X})$ were investigated, where $\mathrm{PMeT}$ was doped with various anions $\mathrm{Y}=\mathrm{CF}_{3} \mathrm{SO}_{3}^{-}, \mathrm{ClO}_{4}^{-}, \mathrm{BF}_{4}^{-}$, $\mathrm{PF}_{6}^{-}$and cadmium sulphide $(\mathrm{CdS})$ doped with various elements such as $\mathrm{X}=\mathrm{Cu}, \mathrm{Ni}$, $\mathrm{Al}, \mathrm{As}$ and $\mathrm{Sb}$. It was shown that the best quality of contact between the organic and inorganic materials was obtained with the $\mathrm{PMeT}\left(\mathrm{PF}_{6}{ }^{-}\right) / \mathrm{CdS}(\mathrm{Sb})$ junction [2.16]. Other researches included deposition of CdSe on PMeT in the presence of silicotungstic acid in the chemical bath to form a p-n type junction, where localized energy levels were created in the forbidden gap of CdSe, which improved the optical properties of the thin films, and hence, the energy conversion efficiency, which increased up to $2.7 \%$ for the Schottky-type photovoltaic organic-organic PMeT/CdSe(STA) photovoltaic junction [2.17]. 


\subsection{PPV}

More recently, due to the rapid advances in organic polymer chemistry, many new polymers with much improved optical and electrical properties have been discovered. One of them is poly (2-methoxy, 5-(2'ethyl-hexyloxy)-pphenylenevinylene) (MEH-PPV). Although MEH-PPV is not the most efficient photovoltaic material, it is well characterized and highly reproducible with a low intrinsic trap density [2.18].

In 1996, Halls et al. [2.19] made a very thorough study on PPV/ fullerene molecule $\mathrm{C}_{60}$ heterojunction. The cell had a fill-factor of 0.48 and an external monochromatic quantum efficiency of $9 \%$. Halls found that the photocurrent spectrum was inversely proportional with the PPV absorption spectrum, indicating that light has to reach the $\mathrm{PPV} / \mathrm{C}_{60}$ interface in order to generate a photocurrent. From the models, an average exciton diffusion length of $6-8 \mathrm{~nm}$ could be derived.

In 2000, Carter et al. [2.20] studied the effect of polymer thickness, hole-mobility and morphology on the device properties of Au/MEH-PPV/TiO $/ 2 \mathrm{TO}$ cell. Flat band energy-level diagrams of the cell are shown in Figure 2.3. They demonstrated that the conversion efficiency in these polymer based photovoltaics was primary limited by the short exciton diffusion length combined with a low carrier mobility. They concluded by presenting a model that describes charge transport in 
solid-state polymer/ $/ \mathrm{TiO}_{2}$ based photovoltaics and suggested methods for improving energy conversion efficiencies in this type of photovoltaic devices.

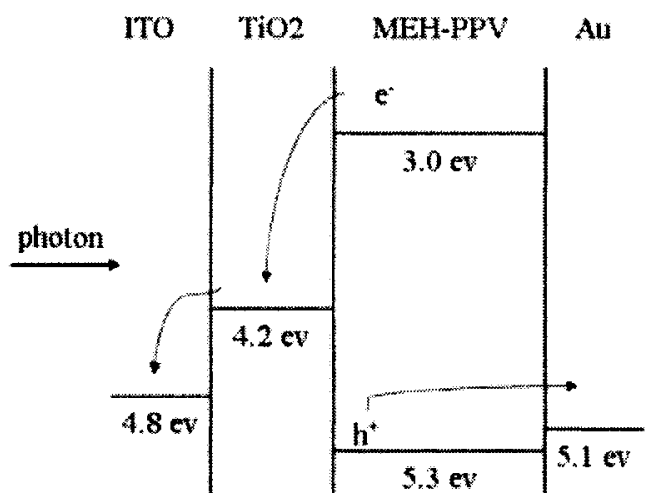

(a)

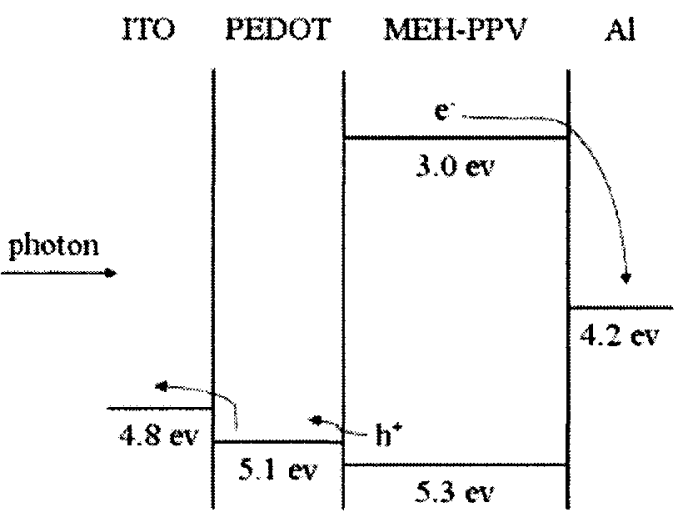

(b)

Figure 2.3: (a) Flat band energy-level diagram for $\mathrm{ITO} / \mathrm{TiO}_{2} / \mathrm{MEH}-\mathrm{PPV} / \mathrm{Au}$ devices. (b) Flat band energy-level diagram for ITO/PEDOT/MEH-PPV/Al devices [2.20].

In all-solid-state multilayer devices, high performance requires good interfacial contact between the layers. Kim et al., in 2005, studied the effect of inserting a PEDOT interlayer between MEH-PPV layer and the $\mathrm{Au}$ electrode of a $\mathrm{TiO}_{2} / \mathrm{MEH}-\mathrm{PPV}$ heterojunctions device on the photovoltaic characteristics of the device [2.21]. The modified device showed improved photocurrent density-voltage characteristics and an increase in the fill factor. They concluded that the improvements were due to the reduction of junction resistance across of MEH-PPV/Au interface in the presence of PEDOT interlayer, which resulted in improved hole injection. 


\subsection{PEDOT:PSS}

Another popular conducting polymer is the polyethylene dioxythiophene (PEDOT): polysterene sulfonic acid (PSS). Solid solutions of PEDOT:PSS form conductive polymer layers which are $p$ doped in their pristine state. Therefore, PEDOT:PSS presents an organic alternative for $p$-type contacts (such as high work function electrodes: ITO, Pt, Au). PEDOT:PSS has a work function of $\sim 5 \mathrm{eV}$, and is known for relatively high transparency, electrical conductance, and easy processing. Charge redistribution in organic/inorganic heterojunctions between PEDOT:PSS and Si was observed by infrared spectroscopic ellipsometry in [2.22].

Yamaura, in 2003 [2.23], also reported a device using an organic-inorganic heterojunctions whose response is highly and selectively sensitive to a UV light. It consists of a p-type semiconducting polymer such as PEDOT:PSS with $E_{g}=1.6 \mathrm{eV}$ and a wide band-gap n-type semiconductor such as a $\mathrm{Nb}$-doped titanium oxide, $\mathrm{TiO}_{2}: \mathrm{Mb}$ or $\mathrm{SrTiO}_{3}: \mathrm{Nb}$. For the PEDOT:PSS/SrTiO3:Nb heterostructure, the photosensitivity at zero bias for the UV light is estimated to be $0.05 \mathrm{~A} / \mathrm{W}$, corresponding to a quantum yield of $16 \%$ electron/photon. This device was observed to exhibit a large response to the UV-B light $(290-320 \mathrm{~nm})[2.23]$.

In $2004, \mathrm{~S}$. Forrest presented a simple non-volatile, write-once-read-many times memory device utilizing an organic on inorganic heterojunctions diode with a 
conductive polymer fuse consisting of PEDOT:PSS. The device switches at 2 and $4 \mathrm{~V}$ for $50 \mathrm{~nm}$ thick PEDOT:PSS on p-type and n-type Si (Au/PEDOT:PSS/Si) respectively, which is significantly lower than the switching voltage used in PEDOT:PSS/p-i-n Si memory elements [2.24]. This was made possible by the ability of PEDOT:PSS to switch under high current densities from a high conducting, stable, p-doped state to a second, non-conducting, or neutral state. 


\subsection{Conclusions}

A brief literature survey on organic on inorganic semiconductor heterojunctions has been conducted. Several combinations of organic materials and inorganic semiconductor materials were described. Refer to Table 2.1. Applications of these organic on inorganic heterostructures include rectifiers, photovoltaic, electroluminescent devices, photodetectors and so on. This chapter will provide a better understanding in the selection of organic and inorganic materials for the organic-on-inorganic semiconductor heterojunction devices to be fabricated.

Table 2.1: Summary of different combinations of organic-on-inorganic heterojunctions with their potential applications

\begin{tabular}{|c|c|c|c|}
\hline Organic Materials & Inorganic Materials & $\begin{array}{c}\text { Some Possible } \\
\text { Applications }\end{array}$ & References \\
\hline PTCDA & Si, GaAs, InP, & Rectifier & {$[2.1,2.9-11]$} \\
\hline PMeT & GaAs, CdSe, CdS, Si & Photovoltaic & {$[2.12-17]$} \\
\hline MEHPPV & $\mathrm{C}_{60}, \mathrm{TiO}_{2}$ & Photovoltaic & {$[2.19-21]$} \\
\hline PEDOT:PSS & $\mathrm{Si}, \mathrm{SrTiO}_{3}: \mathrm{Nb}$ & $\begin{array}{l}\text { UV-detector, WORM } \\
\text { Memory element }\end{array}$ & {$[2.22-24]$} \\
\hline
\end{tabular}




\subsection{References:}

[2.1] Forrest, SR. Kaplan, ML. and Schmidt, PH. (1984) J. Appl. Phys., 55, 1492

[2.2] Cheng, CL. Forrest, SR. Kaplan, ML. Schmidt, PH. and Tell, B. (1986) Appl. Phys. Lett., 47, 1217

[2.3] Dimitrakopoulos, CD. Purushothaman, S. Kymissis, J. Callegari, A. and Shaw, JM. (1999) Science 283, 882

[2.4] Tang, CW. (1986) Appl. Phys. Lett., 48 no. 2, 183

[2.5] Tang, CW. and Vanslyke, SA. (1987) Appl. Phys. Lett., 51, 913

[2.6] Kim, HH. Miller, TM. Westerwick, EH. Kim, YO. Kwock, EW. Morris, MD. and Cerullo, M. (1984) J. of Lightwave Technology, 12, no. 12

[2.7] So, FF. Forrest, SR. Shi, YQ. and Steier, WH. (1990) Appl. Phys. Lett., 56, 674

[2.8] Forrest, SR. (1989) IEEE Circuits and Devices Magazine, 33, May

[2.9] Forrest, SR. Kaplan, ML. and Schmidt, PH. (1984) J. Appl. Phys., 56, 543

[2.10] Forrest, SR. Kaplan, ML. Schmidt, PH. and Gates, JV. (1985) J. Appl. Phys., 57, 2892

[2.11] Forrest, SR. Kaplan, ML. Schmidt, PH. and Parsey, JM. (1985) J. Appl. Phys., 58,867

[2.12] Horowitz, G. and Garnier, F. (1986) Solar Energy Materials and Solar Cells, 13, 47

[2.13] Horowitz, G. and Garnier, F. (1986) Chemtronic, 1, 80

[2.14] Garnier, F. (2002) J. of Opt. A: Pure Appl. Opt., 4, 247

[2.15] Chartier, P. Cong, HN. and Sene, C. (1998) Solar Energy Mat. And Solar Cells, 52,413

[2.16] Cong, HN. Dieng, M. Sene, C. and Chartier, P. (2000) Solar Energy Mat. And Solar Cells, 63, 23 
[2.17] Sene, C. Cong, HN. Dieng, M. and Chartier, P. (2000) Mat. Research Bulletin, 35,1541

[2.18] Arango, AC. Johnson, L. Horhold, H. Schlesinger, Z. and Carter, SA. (1999) Appl. Phys. Lett., 74, 1132

[2.19] Halls, JJM. Pichler, K. Friend, RH. Moratti, SC. and Holmes, AB. (1996) Appl. Phys. Lett., 68, 3120

[2.20] Breeze, AJ. Schlesinger, Z. Carter, SA. and Brock, PJ. (2001) Phy. Rev. B, 64, 125205

[2.21] Song, MY. Kim, K. and Kim, DY. (2005) Solar Energy Mat. \& Solar Cells, 85, 31

[2.22] Schubert, M. Bundesmann, C. and Wemckstern HV. (2004) Appl. Phys. Lett. 84, 1311

[2.23] Yamaura, J. Muraoka, Y. Yamauchi, T. Muramatsu, T. and Hiroi, Z. (2003) Appl. Phys. Lett., 83, 2097

[2.24] Smith, S. Forrest, SR. (2004) Appl. Phys. Lett., Vol. 84, 5019 


\section{Organic on Inorganic}

\section{Semiconductor Heterojunction}

\section{Theory}

\subsection{Overview}

Progress in organic on inorganic semiconductor heterojunction devices requires

the development of a clear understanding of the peculiar electronic physics of the organic material, the inorganic semiconductor and most importantly the junction created between the two materials.

This chapter will first review the organic materials that are commonly found in the fabrication of organic electronic devices, with emphasis given to conjugated polymers. Electronic energy structure and carrier transport in the conjugated polymer will be briefly discussed. The basic principle for inorganic semiconductor will not be mentioned here, as the theory is widely available in any semiconductor physics and devices text. Definition and properties of a heterojunction will be given. Lastly, the possible transport at the interface between the organic on inorganic heterojunction will be explained. 


\subsection{Organic Semiconductor}

Most organic materials are insulators and only those that possess small, finite energy band gaps and moderate amount of carrier concentrations are considered as organic semiconductors. The discovery of doping of polyacetylene to achieve relatively high conductivity reported in 1977 [3.1] lead to a new era of organic electronics. Organic semiconductors are, in general, materials made up of many carbon atoms. The $\mathrm{sp}^{2}$ hybridization in carbon atoms leads to $\mathrm{p}_{\mathrm{z}}$-orbitals, which form $\pi$-bonds. The weak van der Waals forces among the delocalised $\pi$-bonds are the key to the semiconducting properties in some organic materials [3.2]. Since organic semiconductors have a wide range of chemical structures (size, shape, etc.), functionality, crystallinity, morphology, disorder, impurities and etc., the physical properties show a wide variety of behaviour, and often not as simple as in the case of inorganic semiconductor. Usually, organic materials can be classified into 3 main groups: small molecule, oligomers and polymers [3.3]. The main difference between them is their molecular weights, which is reflected by the length of molecular chains. Carbon atoms can form larger molecules or monomers, typically with benzene rings as the basic unit, these are called small molecule organic semiconductors. Small fragments of polymers attached linearly and with a well-defined length ( 2 to 12) are called oligomers [3.4]. On the other hand, polymers are made up of a large number 
$\left(10-10^{3}\right)$ of identical repeating units, all linked together by covalent bonds in a linear fashion. They can be soluble in various solvents, or insoluble when prepared from a precursor route [3.5].

Semiconducting polymers itself can be further categorized into 4 major groups, namely filled polymers, ionically conducting polymers, charge transfer polymers, and conjugated polymers [3.6]. Among these 4 classes of polymers, conjugated polymers are of greatest interest as they have been dominant in the development of plastic electronics since electroluminescence in poly(para-phenlene vinylene) (PPV) was discovered in 1990. Conjugated polymers can act as active semiconducting materials for many semiconductor devices, such as light-emitting diodes, photovoltaic, and transistors. An example of polymers can be simply polyethylene molecule where the repeating monomer unit is $\mathrm{CH}_{2}$ and the end unit is $\mathrm{CH}_{3}$.

\subsubsection{Conjugated polymer}

Polymers are constructed from repeating monomers. The monomers couple with each other to from a polymer chain. These monomers of most polymers are made up of 2 basic elements: carbon and hydrogen atoms. Non-conjugated polymers and conjugated polymers have the same mechanical properties, however due to the different structures of chemical bonds, they have different electronic properties. 
As shown in Figure 3.1(a), the chemical bonds of non-conjugated polymers are formed by $\mathrm{sp}^{3}$ hybrids in the carbon atoms and $1 \mathrm{~s}$ functions in the hydrogen atoms. It requires a large amount of energy to transfer an electron from one bonding orbital to the equivalent antibonding orbital, which means a large energy gap exists between the occupied and unoccupied bands. This large energy gap of the non-conjugated polymer results in the non-conducting electrical properties of most insulators.

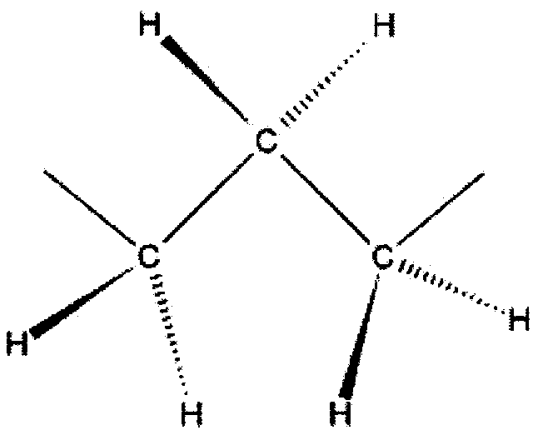

(a)

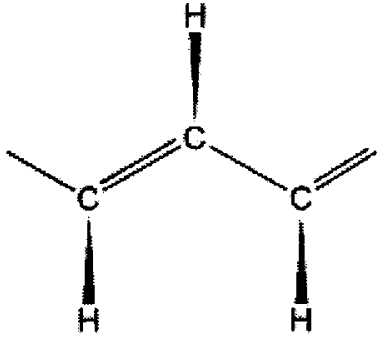

(b)

Figure 3.1: Schematic diagrams of chemical bond structures of (a) non-conjugated polymer and (b) conjugated polymer [3.6]

In contrast, the chemical bond structures of conjugated polymer are different from those of non-conjugated polymers. The distinguishing feature of all conjugated polymers is the unsaturated carbon-based alternating single and double bond structure of the polymer backbone, or the so-called conjugated carbon chain. As shown in Figure 3.1(b), parts of chemical bonds are formed by $\mathrm{sp}^{2}$ hybrids in the carbon atoms and $1 \mathrm{~s}$ function in the hydrogen atoms. Only three of the four valence electrons of a 
carbon participate in this $\alpha$-backbone. This leaves one remaining electron per carbon atom, which is located perpendicular to the trigonal plane in a $p_{z}$ orbital. All these leftover $p_{z}$ orbital electrons from adjacent carbons overlap to form a $\pi$-system. This can be described as a delocalized $\pi$-electron cloud with a periodic alternating density. It requires less energy for an electron to be transferred from a $\pi$ bonding to an equivalent antibonding orbital. Thus, the energy gap between occupied and unoccupied bands in conjugated polymer is relatively smaller than that of non-conjugated polymer. The typical gaps are in the range of $1 \sim 3 \mathrm{eV}$. Figure 3.2 shows several examples of conjugated polymers with alternating single and double bonds in the chain.<smiles>C=CC(C)(C)/C=C/C(C)(C)/C=C/C</smiles>

poly(acetylene) PA<smiles>CC(C)(c1cccs1)c1ccc(C(C)(c2cccs2)c2cccs2)s1</smiles>
poly(thiophene) PT

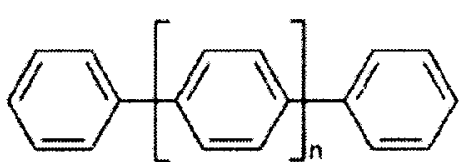

poly(para-phenylene) PPP

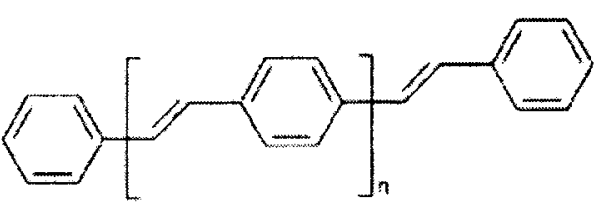

poly(para-phenylene-vinylene) PPV

Figure 3.2: Several examples of conjugated polymers (shown by the sequence of alternating single and double bonds in the polymer). [3.6] 


\subsubsection{Electronic energy structure of conjugated polymer}

As mentioned above, a conjugated system is one featuring an alternation between

single and double bonds. The essential property which comes from conjugation is that the $\pi$ electrons are much more mobile than the $\sigma$ electrons. This means that the $\pi$ electrons can jump from site to site between carbon atoms with a low potential energy barrier as compared to the ionization potential. The $\pi$ electron system shows all the essential electronic features of organic materials, such as light absorption and emission, charge generation and transport.

Each carbon atom in a conjugated system has 3 nearest neighbours with which it forms 3 equivalent $\sigma$ bonds made from the trigonal $\mathrm{sp}^{2}$ hybridization of 3 valence atomic orbitals of the carbon atoms: $2 \mathrm{~s}, 2 \mathrm{p}_{\mathrm{x}}$ and $2 \mathrm{p}_{\mathrm{y}}$ for example. For such a hybridization state, the fourth orbital $2 \mathrm{p}_{z}$ lies perpendicular to the $\sigma$ bond plane. It is this lateral overlap of these out-of-plane $2 p_{z}$ atomic orbitals which gives the $\pi$ bonds. A schematic diagram of the orbitals for two $\mathrm{sp}^{2}$ hybridized carbon atoms is shown in Figure 3.3. It is the mutual overlap of the $\pi$-orbitals on neighbouring atoms in the polymer chain that causes the wave functions to delocalise over the conjugated polymer and form the delocalised $\pi$-system. 


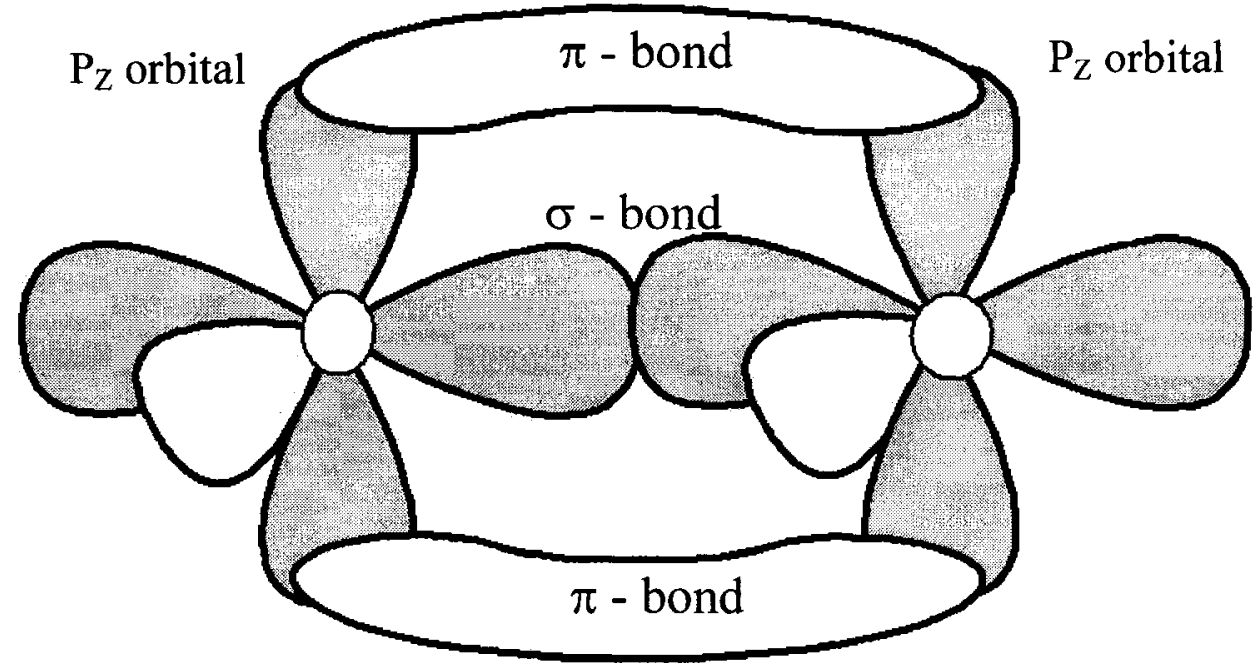

Figure 3.3: 3D Schematic diagram of the orbitals for two $\mathrm{sp}^{2}$-hybridized carbon atoms

The electronic properties of polymers can be described in terms of semiconductor physics. Polymers are bonded by strong covalent bonds. Peierls' distortion predictions explain the development of two molecular bands, namely the $\pi$-band originating from the Highest Occupied Molecular Orbital (HOMO) and $\pi^{*}$-band originating from the Lowest Unoccupied Molecular Orbital (LUMO), with an energy gap in-between. The 1D-band that results has considerable band-width (on the scale of an $\mathrm{eV}$ ). As the $\pi$-orbital overlap is weaker than the $\sigma$-orbital overlap, the energy band-gap between the bonding and antibonding molecular orbitals is smaller for the $\pi-\pi^{*}$ case than for the $\sigma-\sigma^{*}$ one. Thus, one can limit the band study to the $\pi-\pi^{*}$ molecular orbitals. The $\sigma$-bonds then only contribute to the stability of the molecular structure [3.7]. The gaps between occupied and empty states in these delocalized $\pi$-systems become smaller with increasing delocalization along the polymer chain. 
The presence of the alternating single and double bonds is responsible for the unique properties of conjugated polymers. Take for example polyacetylene, $\left(\left(\mathrm{C}_{2} \mathrm{H}_{2}\right)_{n}\right)$, the simplest conjugated polymer chain, each carbon atom in the chain has one unpaired electron (electron configuration $\mathrm{sp}^{2} \mathrm{p}_{z}{ }^{1}$ ). The energy levels of polyacetylene depend on the value of $n$. As $n$ increases, the polymer chain is longer and thus the $\pi$-system becomes more delocalised. Therefore, with increasing $\mathrm{n}$, the gap between occupied $\pi$ and unoccupied $\pi^{*}$ states becomes smaller. For example, when $n$ is infinite and every orbital is occupied by one electron due to the uniform bond order, the polymer should have a similar energy structure as an intrinsic metal. When the band is half-filled, bond dimerization occurs as the result of Peierls' distortion and the system becomes a semiconductor with a small band gap [3.1]. The energy structure of polyacetylene is shown in Fig. 3.4. The valence effective Hamiltonian (VEH) method can be used to calculate the electronic energy structure for conjugated polymers. The basic parameters include the band-gap energy, the bandwidth, ionization potential, and electron affinity. Using the dimensionless characteristic parameter $\lambda=2 \alpha^{2} / \pi \mathrm{t}_{0} \kappa$ and bandwidth $\mathrm{W}=4 \mathrm{t}_{0}$, the band gap $\left(\mathrm{E}_{\mathrm{g}}=2 \Delta_{0}\right)$ is given by

$$
\Delta_{0}=2 \mathrm{~W} \exp \left[-\left(1+\frac{1}{2 \lambda}\right)\right]
$$

where $t_{0}$ is the inter carbon transfer integral for $\pi$-electron, $k$ is the effective spring constant, and $\alpha$ is the lattice constant [3.1]. 


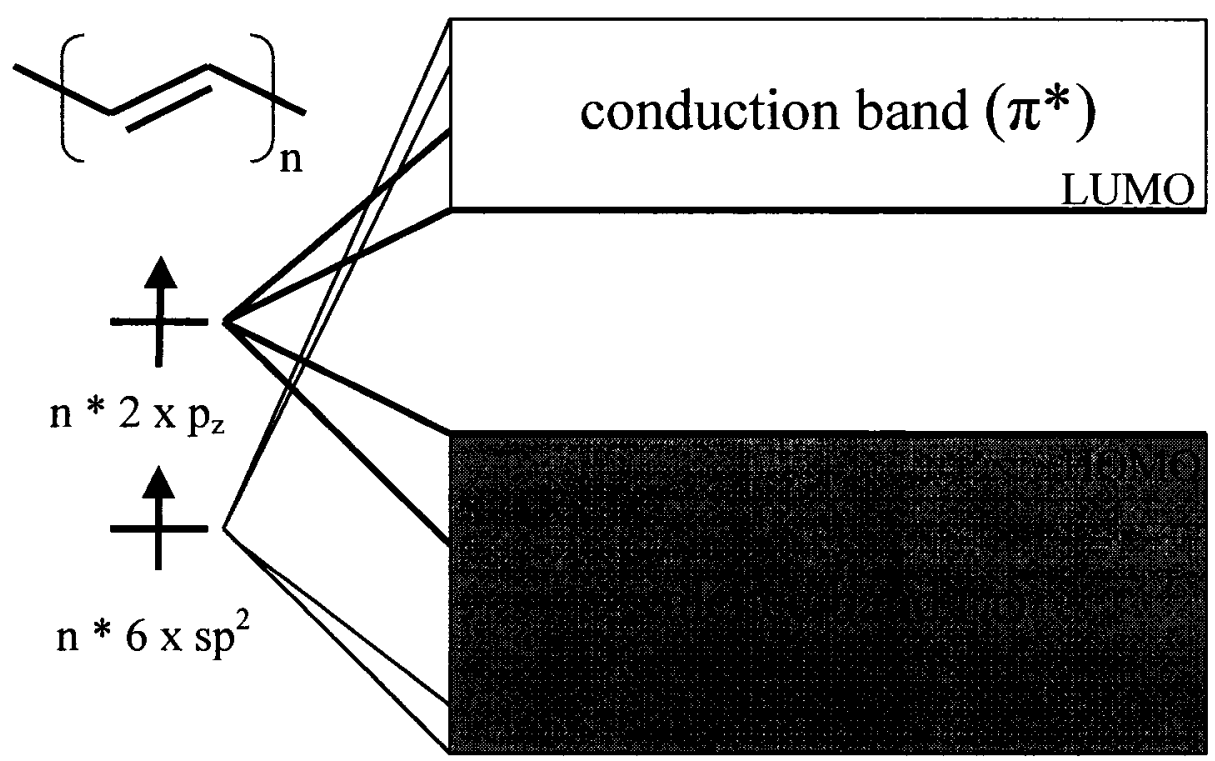

Figure 3.4: Schematic diagram of the energy structure for poly(acetylene) [3.2]

\subsubsection{Electron transport in conjugated polymer}

Transport and mobility in organic materials require knowledge of the charged species. In conjugated polymers, due to the intrinsic low dimensionality of the system, charge carriers tend to have a strong coupling to the lattice. This factor together with electron-electron correlation effects and other molecular structural details can lead to the formation of various types of excitation in conjugated polymers [3.8]. For example, solitons (in degenerate ground state structures like polyacetylene), polarons and bipolarons (in nondegenerate ground state structures like polypyrrole, etc.), bound excitons (in strongly correlated systems like polydiacetylene, etc.), electrons and holes (injected carriers in semiconducting polymer devices) and free carriers (in fully doped metallic conjugated polymers) [3.9]. The charge transport mechanism in 
conducting polymer is quite complex compared with conventional systems due to the various morphological factors (e.g. crystallinity, amorphous nature, intermolecular interactions, etc.), degree of disorder (both intra- and inter-chain) and the wide range of charge carrier species. In general, the conjugated length (extent of the delocalized $\pi$-electrons in the polymer backbone), interchain interactions, carrier density and the degree of disorder are the most significant parameters [3.9], playing major roles in the charge transport mechanism in conducting polymers.

Polarons may be regarded as defects in conjugated polymer chain or charged quasi-particles which induce a lattice deformation. Such a defect stabilizes the charge, which is thus self-trapped as a consequence of lattice deformation.

Excitation of the polymer creates one electron and a hole on the chain. This effect is particularly important when the electron-hole interactions are strong. Coulomb attraction keeps them together and the two opposite charges are considered as a bound electron-hole pair. An exciton is named according to its delocalization. If it is localized on one molecular unit, it is called a Frenkel exciton. If the exciton extends over many molecular units, it is a Mott-Wannier type of exciton. [3.9]

Bipolarons are double charged carriers where a strong interaction with the lattice (electron-phonon interaction) can lead to a stabilization of two charges despite the Coulomb repulsion. 
The bulk conductivity of conducting polymers should consist of contributions, at least, from the intrachain and interchain electron transportations. While the intrachain diffusion of the charge carriers along the conjugated backbone plays a dominant role in the charge transporting process, the interchain transport (via hopping, tunnelling, etc) between adjacent polymer chains also has a significant influence on the bulk conductivity since a single chain does not extend throughout the entire length of a sample in most practical applications. The former, which is specific to conjugated polymers, is the most efficient. As the extent of disorder increases, hopping transport dominates. Therefore, improving interchain/molecular transport and reducing the extent of disorder holds the key for enhancing charge transport properties like mobility, mean free path, etc, in organic semiconductors. [3.9] 


\subsection{Heterojunction}

A heterojunction is a junction formed between two dissimilar semiconducting materials often with unequal bandgaps. Both materials contribute to the charge transport and carrier concentration. On the other hand, if the semiconductor material is the same throughout the entire structure, this type of junction is referred to as a homojunction. Usually, the energy bandgap of an organic material (defined by the difference between HOMO and LUMO) and that of an inorganic semiconductor material (defined by the difference between conduction and valence bands) are unequal and non-matching. Therefore, the junction formed by a p-type organic polymer and an n-type inorganic semiconductor is a heterojunction.

Since the two materials used to form a heterojunction have different energy bandgaps, the energy band will have a discontinuity at the junction interface. We may have an abrupt junction in which the semiconductor changes abruptly from a narrow-bandgap material to a wide-bandgap material. For inorganic semiconductors, in order to have a useful heterojunction, the lattice constants of the two materials must be well matched. Any lattice mismatch can introduce dislocations resulting in interface states [3.10]. However, the organic-inorganic semiconductor interface is still under extensive studies. Usually the semiconductor will serve as substrate for the organic film. The first monolayer of the conjugated organic material will have certain 
interaction with the substrate material, defining the alignment between the observable substrate valence band (semiconductor) and the molecular orbitals of the absorbed organic material [3.11]. This is true only if the interaction between substrate and conjugated organic material is relatively weak and does not lead to a chemical decomposition of the organic molecule. There are already some publications on the organic-metal interfaces measured using photoemission spectroscopy techniques.

Formation of heterojunction instead of homojunction is very common in organic semiconducting devices due to two main reasons. Firstly, the relatively low carrier concentration in organic semiconductors makes homojunction organic devices practically inefficient in term of device performance. Secondly, the vast variety of organic semiconducting materials allows a large degree of material matching which may lead to momentous improvements in overall device performance or for specific applications. 


\subsection{Organic - Inorganic Semiconductor Interface}

Electronic devices invariably rely on the properties of interfaces between electrical conductors. The successful application of conjugated polymers to electronic devices such as light-emitting diodes, solar cells or transistors requires an understanding of charge transport at their interfaces. Transport at electroactive interfaces is fundamentally different from transport in bulk media. One primary reason is that the bulk of a uniform conductor cannot contain excess charges at equilibrium or steady-state. Interfaces, however, are a seat for excess charges, and this can have a strong influence on the transport. The transition in electronic structure at interfaces also adds complexity, providing a source of asymmetry that is not observable with transport through uniform bulk materials. The author in [3.12] gave a detailed theoretical interpretation of the charge transport at conjugated polymer-inorganic semiconductor and conjugated polymer-metal interfaces, which will be briefly described below.

The most comprehensive picture of the electronic structure of a material is its density of states diagram. For conjugated polymers, the two key energies are the LUMO and HOMO energies in a molecular model. In the energy level diagrams, the electronic structure of the polymer is simply indicated by the two characteristic 
energies: ionization energy $\left(I_{p}\right)$ and electron affinity $(\chi)$. For conjugated polymer, the ionization energy is defined as the energy difference between the vacuum level (VL) and the leading edge of the HOMO peak, while energy separation between LUMO and VL corresponds to electron affinity. The energy gap of the conjugated polymer $\left(E_{\mathrm{gP}}\right)$ is defined as the energy difference between LUMO and HOMO. Conjugated polymer work function $\left(\phi_{\mathrm{P}}\right)$ corresponds to the energy difference between the Fermi level and the VL. Furthermore, these energy levels are drawn spatially extended, suggesting band-like structure. These bands, however, can be easily replaced with a string of localized electronic states. For inorganic semiconductor, the bang-gap energy $\left(E_{g I S}\right)$ is defined as the energy difference between the energies at conduction band $\left(E_{c}\right)$ and valence band $\left(E_{v}\right)$. The parameter $\phi_{I S}$ is the semiconductor work function.

To have a better theoretical understanding of the inorganic semiconductor and conjugated polymer interfaces, the representative band-bending diagram must be studied [3.12]. Figure 3.5 and 3.6 show the diagrams expected for an ideal interface between p-type conjugated polymer and an n-type inorganic semiconductor in equilibrium before and after contact. For a long time, a naive model had often been applied to estimate the interfacial electronic structure of organic devices. This traditional model is in analogy to the Mott-Schottky model, which is a widely known textbook model for inorganic semiconductor/metal interfaces. For an ideal interface, 
given by the famous Mott-Schottky relation originally proposed for inorganic-metal interfaces $[3.13,3.14]$, the barrier height for hole (or electron) injection, $\phi_{B}^{p}\left(\phi_{B}^{n}\right)$ can be expressed as

$$
\begin{aligned}
& e \phi_{B}^{p}=E_{V}-e \phi_{P} \\
& e \phi_{B}^{n}=e \phi_{P}-E_{C}=e \phi_{P}-\left(E_{V}-E_{g I S}\right)=E_{g I S}-\phi_{B}^{p}
\end{aligned}
$$

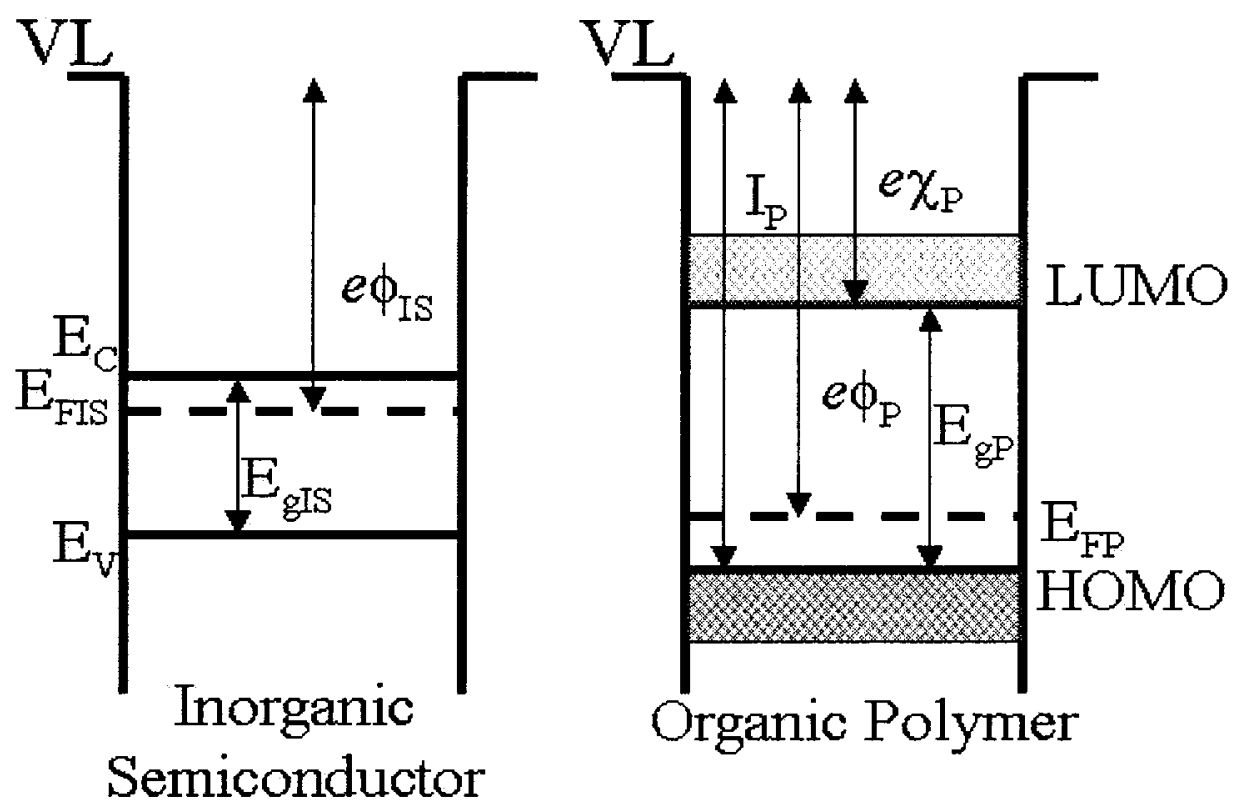

Figure 3.5: Energy band diagram for an inorganic semiconductor and conjugated polymer before contact [3.12]. 


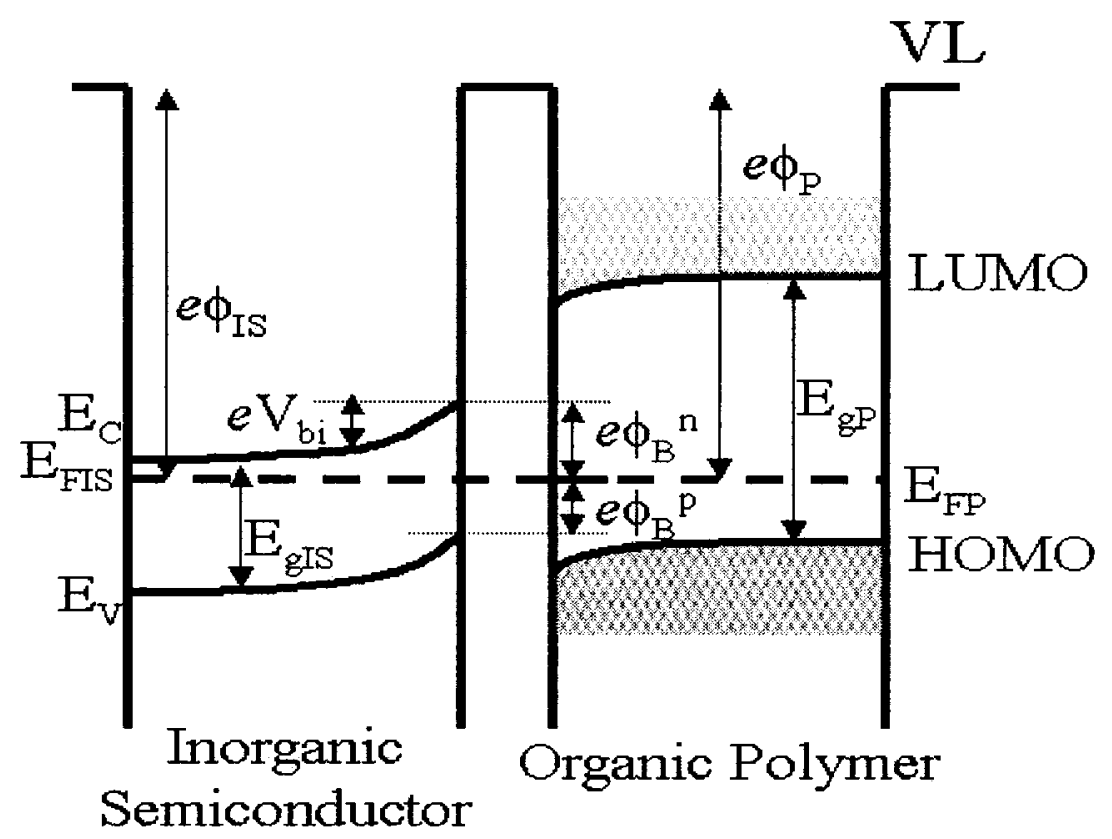

Figure 3.6: Energy band diagram for an inorganic semiconductor and conjugated polymer after contact using Mott-Schottky model [3.12].

However, the authors in [3.15] have proven the invalidity of the assumption of a common vacuum level during the energy level alignment of organic semiconductor/metal interface. The breakdown of the assumption of a common vacuum level means that the vacuum level does not align but shifts at the organic/metal interfaces. Thus, the assumption of a common vacuum level should not be valid in the case of inorganic semiconductor/conjugated polymer energy level alignment as well. The deviation of the vacuum levels for the organic and inorganic semiconductor is called vacuum level shift, $\Delta$. This "shift" is hypothetical: the concept of vacuum level does not make sense at the interface because there is no vacuum space where a VL is defined [3.16]. When $\Delta$ is taken into account, the key equilibrium 
parameter barrier height for hole (or electron) injection, $\phi_{B}^{p}\left(\phi_{B}^{n}\right)$ as shown in Figure 3.7 can be expressed as

$$
\begin{aligned}
& e \phi_{B}^{p}=E_{V}-\left(e \phi_{P}+\Delta\right) \\
& e \phi_{B}^{n}=e \phi_{P}+\Delta-E_{C}=e \phi_{P}+\Delta-\left(E_{V}-E_{g I S}\right)=E_{g I S}-\phi_{B}^{p}
\end{aligned}
$$

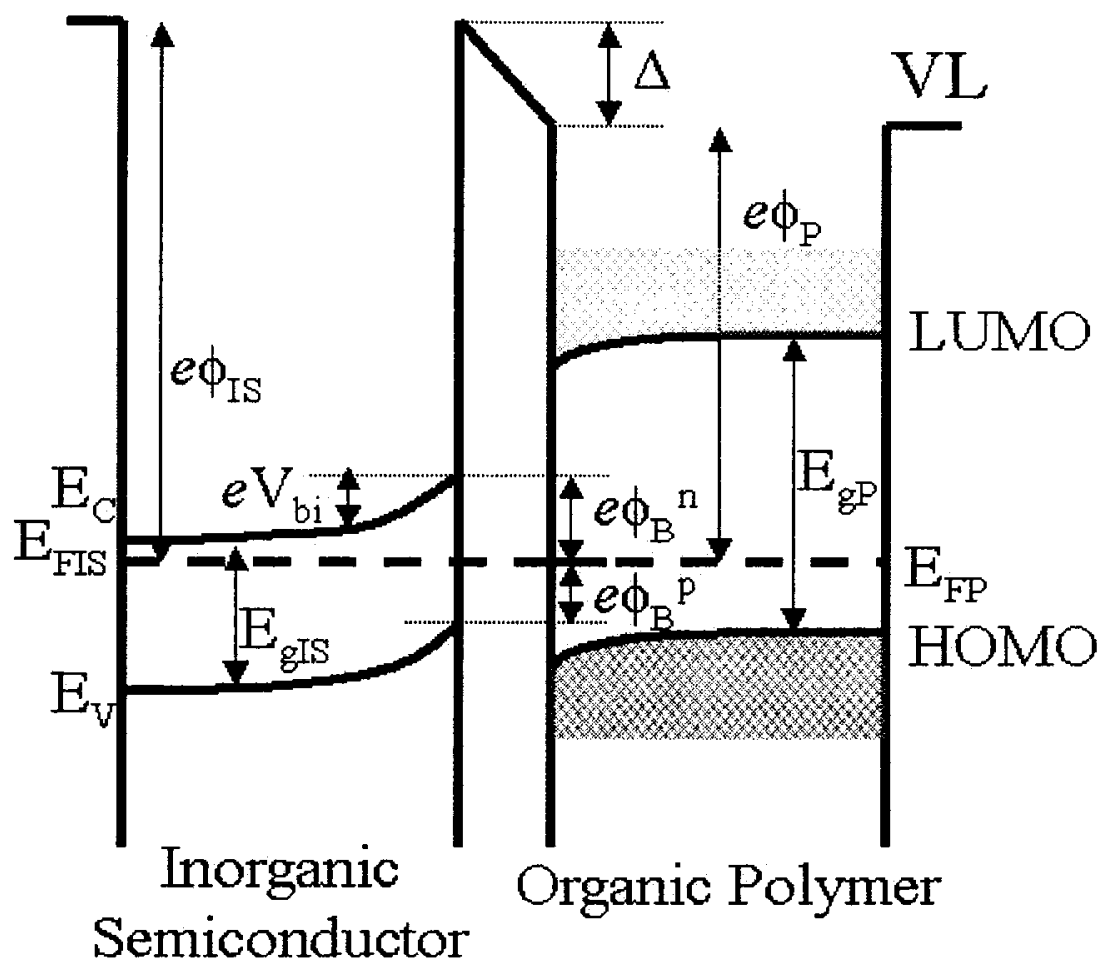

Figure 3.7: Energy band diagram for an inorganic semiconductor and conjugated polymer after contact using vacuum level shift model [3.12].

Many research groups have carried out systematic studies of $\Delta$ for various combinations of organics/metals and organic/inorganic semiconductor interfaces.

Generally, the slope parameter defined as

$$
S=\frac{d \phi_{B}^{n}}{d \phi_{P}}
$$

is used for characterizing the relationship between the barrier height and Fermi energy. 
It is known that $\mathrm{S}$ depends on the type of the semiconductor [3.16]. Since $e \Phi_{B}^{n}$ can be expressed as $e \Phi_{B}^{n}=e \Phi_{P}+\Delta-\left(E_{V}-E_{g I S}\right)$, therefore,

$$
S=1+\frac{d \Delta}{d \phi_{P}}
$$

indicating that the slope of VL shift against $\Phi_{p}$ corresponds to S-1.

When Mott-Schottky rule holds, i.e., there is no interface state at the interface (Schottky limit), $\Delta=0$ and the value of $S$ should be unity. On the other hand, when there is interface state with sufficient density to pin the Fermi level of the semiconductor, S should be zero (Bardeen limit) [3.11]. The authors in [3.17] have shown that $S=0.4$ for InP-PPy:PMo12 interface. Many other research groups have observed results similar to the ones described above although much less than the Schottky-Mott limit, $\mathrm{S}$ is much greater than the value of 0.1 for Si/metal interface.

Many prominent theories of Fermi-level pinning consider the presence of some form of interface states $[3.18,3.19]$. As in Bardeen's original model [3.20], these interface states provide a source of charges that participates in charge transfer equilibrium. As these states reside at the immediate interface, their charging will result in a rigid band shift, thereby altering the effective barrier height. The explanation of Fermi-level pinning using interface states requires that the states depend only on the inorganic semiconductor. In other words, most interface state models of Fermi-level pinning should hold regardless of the nature of contact, 
whether metal or polymer [3.12].

In regard to transport at inorganic semiconductor - conjugated polymer interfaces, nearly free-electron thermionic emission theory has been generally assumed. Thermionic emission considers the thermal activation of free electrons over a barrier height $\phi_{\mathrm{B}}$ much larger than $\mathrm{kT}$, so that Maxwell-Boltzmann approximation applies [3.21]. The incident electrons are assumed to cross with unit probability if they have sufficient energy to surmount the potential barrier and with zero probability otherwise.

The characteristic feature of inorganic semiconductor interfaces that makes them useful in microelectronic is the current rectification, or diode behaviour. The $\mathrm{J}-\mathrm{V}$ relation for many inorganic semiconductor interfaces qualitatively follows the phenomenological diode equation:

$$
J=J_{S}\left[\exp \left(\frac{e V_{a}}{k_{B} T}\right)-1\right]
$$

where $J_{S}$ is voltage-independent constants; $V_{a}$ is the applied voltage; $\kappa_{B}$ is the Boltzmann's constant; and T is the temperature. This is also known as the ideal-diode equation, gives a good description of the current-voltage characteristics of the junction.

An important parameter for characterizing any diode is the rectification ratio:

$$
R R(V)=|J(V) / J(-V)|
$$


The ideality or quality factor, $\eta$, used to describe the strength of the exponential increase in current with applied forward bias is given as:

$$
\eta=\frac{\kappa T}{e}\left(\frac{d \ln J}{d V}\right)^{-1}
$$

Under reverse bias, the saturation current in the organic layer $\left(\mathrm{J}_{\mathrm{os}}\right)$ is given by [3.22]:

$$
J_{o s}=-A^{*} T^{2} \exp [-q(\phi B-\Delta \phi) / \kappa T]
$$

where $A^{*} \equiv \frac{4 \pi e m_{n}^{*} \kappa^{2}}{h^{3}}$ is the Richardson constant, $\Delta \phi$ is the image force barrier lowering given by $\Delta \phi=\sqrt{q E_{m} / 4 \pi \kappa_{I}}, \kappa_{\mathrm{I}}$ is the permittivity of the inorganic substrate, and $E_{m}$ is the electric field in the substrate. Requiring continuity of both hole and electron currents leads to the familiar expression for thermionic emission across the organic-inorganic semiconductor barrier:

$$
-J=J_{o s}\left\{\exp \left[-q\left(V_{a}-V_{P}-V_{R}\right) / n \kappa T\right]-1\right\}
$$

where $\mathrm{n}$ is the diode ideality factor, $\mathrm{V}_{\mathrm{a}}$ is the applied voltage such that

$$
V_{a}=V_{D}+V_{P}+V_{R}
$$

In Equation 3-11, $V_{D}$ is the voltage drop across the inorganic semiconductor, $V_{P}$ is the voltage drop across the conjugated polymer and $V_{R}$ is the total voltage across the device series resistance $R$.

It is questionable whether a free-electron approach will be valid at interfaces between inorganic semiconductor and conjugated polymer, given their disparate 
electronic structures and the possibility for strong localization in the polymer [3.23]. Many of the barrier heights reported at the inorganic semiconductor/polymer interfaces are simply effective barrier heights related to the magnitude of the current but not necessarily the true barrier height at the interface. 


\subsection{Conclusions}

In this chapter, the organic semiconductor materials have been introduced including their classifications: small molecules, oligomers and polymers. Conjugated polymers present electrical properties similar to the inorganic semiconductor due to the $\mathrm{sp}^{2}$ hybridization of the carbon atoms. The alternating single and double carbon bondings give rise to their semiconducting properties. The delocalised $\pi$-system in the conjugated polymer was used to explain its electronic energy structure formed by the HOMO and LUMO with an energy band-gap in between. The charge transport mechanism in conducting polymer was later described. A brief description on the formation of a heterojunction was also given. Lastly, the transport across the organic-inorganic semiconductor interface was discussed where the thermionic emission theory has been generally assumed to be valid for this interface. 


\subsection{References:}

[3.1] Tanabe, Y, (1999) Macro-molecular Science and Engineering, New Aspects, Springer: New York, U.S., 319

[3.2] Leo, K. (2005) Organic Semiconductors: The Basics, Organic Semiconductor World, http://www.iapp.de/orgworld/index.php?order=2,1\&lan=en.

[3.3] Natali, D and Sampietro, M, (2003) Nuclear Instruments and Methods in Physics Research A 512, 419-416.

[3.4] Mullen, K. and Wegner, G, (1998) "Electronic materials: the oligomer approach," Wiley-VCH Weinheim.

[3.5] Marshall. JM, and Dimova-Malinnovska, D., (2002) "Photovaltaic and Photoactive Materials - Properties, Technology and Applications," NATO Science Series

[3.6] Bing R. Hsirh and Yen Wei, Editors, (1999) Semiconducting Polymers, American Chemical Society: Washington, DC, U.S., 1.

[3.7] Su, PW. Schrieffer, JR. and Heeger, AL, (1979) Phys Rev Lett 42, 1698

[3.8] Emin, D. (1996) in, "Handbook of conducting polymers," Dekker, M., Vol 2, Ch. 26

[3.9] Brabec, C. Dyakonov, V. Parissi, J. and Sariciftci, NS. (2003) "Organic Photovolaics Concepts and Realization," Springer

[3.10] Monch W. (1995) "Semiconductor Surface and Interfaces," $2^{\text {nd }}$ ed. Berlin: Springer-Verlag.

[3.11] Salaneck, RS. Seki, K. Kahn, A. Pireasux, JJ. (2002) "Conjugated Polymer and Molecular Interfaces" Marcel Dekker, Inc New York [3.12] Lonergan, M. (2004) Annu. Rev. Phys. Chem 55, 257-98

[3.13] Schottky, W. (1939) Z. Physik. 113, 367-414 
[3.14] Mott, NF. (1939) Proc. R. Soc. 171, 27-38

[3.15] Narioka, S. Ishii, H. Yoshimura, D. Sei, M. Ouchi, Y. Seki, K. Hasegawa, S.

Miyazaki, T. Harima, Y. and Yamashita, K. (1995) Appl. Phys. Lett. 67, 1899-1901

[3.16] Ishii, H. and Seki, K. (2002) "Energy Level alignment at organic-metal interfaces," in "Conjugated Polymer and Molecular Interfaces," M. Dekker, Inc. New York

[3.17] Daniels-Hafer, C. Jang, M. Boettcher, SW. Danner, RG. Longergan, MC. (2002) J. Phys. Chem. B 106, 1622-36

[3.18] Henisch, HK. (1984) "Semiconductor Contacts." Oxford: Clarendon. 377

[3.19] Rhoderick EH. and Williams RH. (1988) "Metal-semiconductor Contacts." Oxford: Clarendon. 252

[3.20] Bardeen J. (1947) Phys. Rev. 71, 717-27

[3.21] Neamen, DA. (1998) "Semiconductor Physics and Devices: Basic Principle," $\operatorname{Irwin} 2^{\text {nd }}$ ed. 319

[3.22] Forrest, SR. Kaplan, ML. and Schmidt, PH. (1984), J. Appl. Phys 55(6), $1492-1507$

[3.23] Kumar, A. Wilisch, WCA. and Lewis, NS. (1993) Crit. Rev. Solid State Mater. Sci. 18, 327-53 


\section{Fabrication of Organic on}

\section{Inorganic Semiconductor}

\section{Heterojunction}

\subsection{Overview}

The two most common techniques for organic thin film deposition are vacuum deposition and solution coating which are dependent on the choice of organic materials. Materials with good thermal stability are required for vacuum evaporation while materials for solution processing need to be soluble. Small molecular organic materials may be thermally more stable but less soluble than polymers, where good solubility often is achieved by side-chain solubilization [4.1]. Polymers will decompose at high temperatures and have a too large molecular mass for evaporation. Hence for small molecules, evaporation is the best choice, whereas solution processing is often used for the semiconducting polymers.

\subsubsection{Evaporation}

The vacuum deposition is often used to prepare very thin organic films (a few hundred $\dot{A}$ or less) [4.2] at a pressure of $<10^{-5}$ Torr. Thus the mean free path of the evaporated molecule $(>450 \mathrm{~cm}$ ) is usually longer than the distance between the 
evaporation source and the target $(\sim 15 \mathrm{~cm})$. In addition, contaminants like oxygen and water are reduced therefore resulting in better film quality and higher device performance. However, evaporation is a time-consuming and expensive process.

\subsubsection{Wet processing}

Common to all wet processing techniques is the solubility of organic materials in an appropriate solvent like water or any other polar or non-polar organic solvent. A special case is the solution processing of a soluble monomer coupled with a polymerization reaction during (e.g. electrochemical polymerization) or after heat treatment [4.1]. This has the advantage that after preparation, the resulting polymers are insoluble and another film can be deposited from another solution on top of them. If polymer blends are directly processed from a solution, several common techniques can be applied and spin-coating is the most popular method. Spin-coating is fast and economical. However, it requires a very clean environment to ensure acceptable level of device yield and performance. The relevant parameters for this technique include the choice of substrate, the solvent, concentration of the organic polymers in the solvent, the substrate temperature and most importantly the spin coating speed.

\subsubsection{Different configurations}

Over the decade, several research groups mentioned in Chapter 2 have been working on the organic on inorganic semiconductor heterojunctions and a number of 
device structures have been proposed. They include: a) structures with two backside electrodes and b) sandwich devices.

For the two backside electrodes structure, inorganic semiconductor and organic polymer are both deposited one after another onto a separate substrate such as glass or plastic plate. Electrodes are then deposited onto the two materials respectively with the help of photolithography. Advantage of this structure is that the device can be fabricated on any substrate. Sandwich structure on the other hand is the most common device configuration that has been used in studying the organic/inorganic semiconductor junction. In this case, the inorganic semiconductor will act as the substrate with a metal contact deposited on the back side of the substrate. The organic material can then be evaporated or spin-coated onto the front side of the inorganic substrate. A front electrode deposited on top of the organic polymer will complete this diode fabrication. The front electrode may or may not cover the whole organic polymer. Advantage of this configuration is that the process only consists of a few fast and simple steps. 


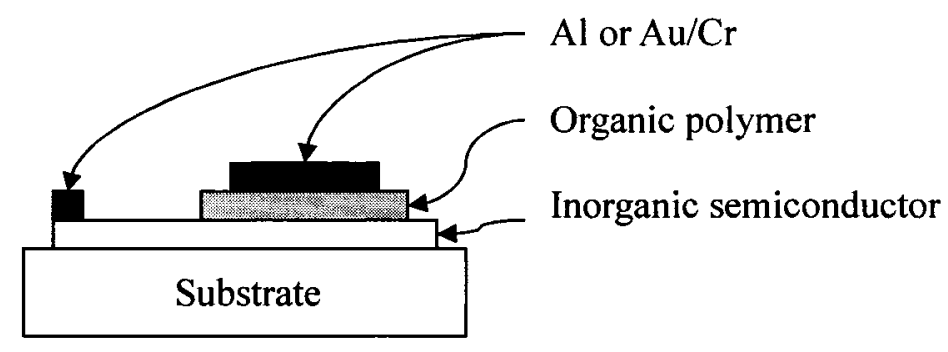

(a)

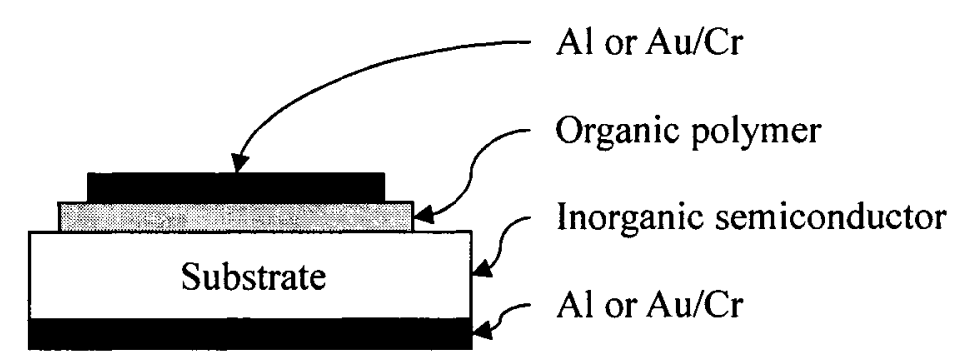

(b)

Figure 4.1: Possible device geometries for hybrid devices: (a) structure with two backside electrodes and (b) sandwich device. [4.3] 


\subsection{Experimental Setup}

In the present experiments, the fabrication of organic on inorganic semiconductor heterojunction devices involves a number of facilities that are commonly found in any semiconductor fabrication lab. A brief description of some of the facilities will be given in the following sections. These facilities include an evaporation system, a spin coat processor and a mask-aligner. An understanding of the operational principle of the equipment will provide the readers with a better picture of the whole fabrication procedure.

\subsubsection{Thermal Evaporation System}

Resistive thermal evaporation is one of the most commonly used metal deposition techniques. It consists of vaporising a solid material (pure metal or compound) by heating it to sufficiently high temperatures and recondensing it onto a cooler substrate to form a thin film. As the name implies, the heating is carried out by passing a large current through a filament container (usually in the shape of a boat or crucible) which has a finite electrical resistance. The choice of filament is dictated by the evaporation temperature and its inertness to alloying/chemical reaction with the evaporant [4.4]. This technique is also known as indirect thermal evaporation since a supporting material is used to hold the evaporant.

Once the metal is evaporated, its atoms undergo collisions with the surrounding 
gas molecules inside the chamber. As a result, a fraction is scattered within a given distance during their transfer through the ambient gas. The mean free path in air at $25^{\circ} \mathrm{C}$ is approximately 45 and $4500 \mathrm{~cm}$ at pressure of $10^{-4}$ and $10^{-6}$ Torr respectively. Therefore, pressure lower than $10^{-5}$ Torr is necessary to ensure a straight line path for most of the evaporated metal species and for the substrate-to-source distance of approximately 10 to $50 \mathrm{~cm}$ in a vacuum chamber. Hence, good vacuum is a prerequisite for producing contamination free deposits [4.5].

In this work, the thermal evaporation of gold as the top electrode for the device is performed using an Edwards model E306A Coating System. The vacuum system is equipped with one high-current source for thermal evaporation. The pressure of the vacuum chamber is able to reach less than $1 \times 10^{-6}$ Torr, which is sufficient for uniform metal film deposition.

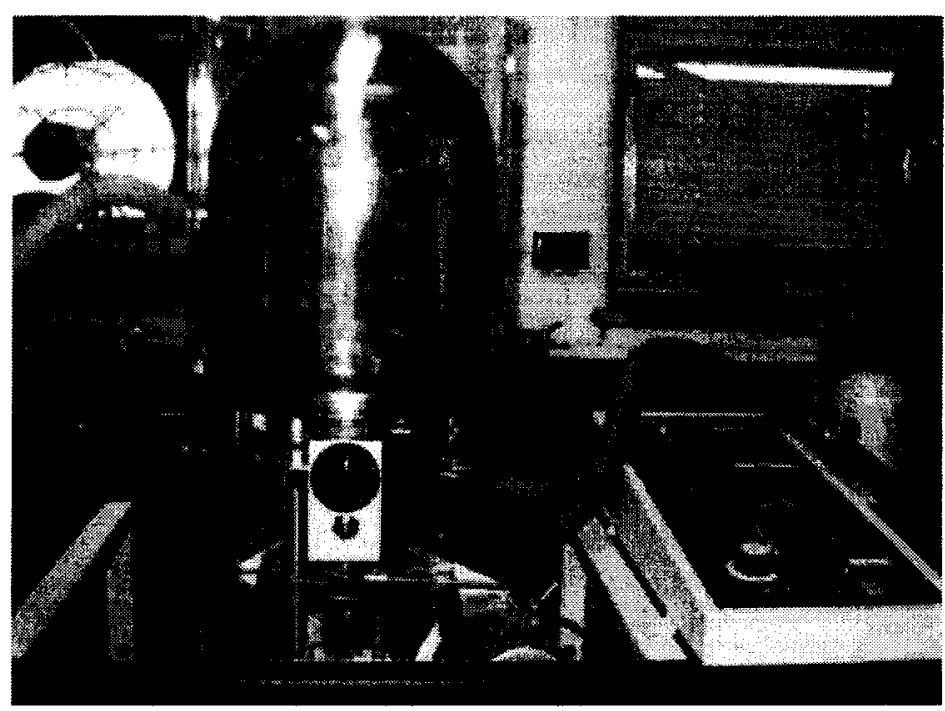

Figure 4.2: Edwards model E306A Coating System. 


\subsubsection{Spin-coat Processor}

Spin coating has been used for several decades for the application of solvent-based thin films. A typical process involves depositing a small amount of fluid (resin, organic polymer, etc) onto the center of a substrate and then spinning the substrate at high speed (typically around $1000-6000 \mathrm{rpm}$ ). Centripetal acceleration will cause the fluid to spread to and eventually off the edge of the substrate leaving a thin film of the fluid on the surface. Final film thickness and other properties will depend on the nature of the resin (viscosity, drying rate, percent solids, surface tension, etc) and the parameters chosen for the spin process [4.6]. Factors such as final rotational speed, acceleration and spin time contribute to the properties of coated films. The final film thickness is generally determined by the combination of spin speed and time.

One of the most important factors in spin coating is repeatability. Subtle variations in the parameters of the spin process can result in drastic variations in the coated film.

In general, higher spin speeds and longer spin times create thinner films. The spin coating process involves a large number of variables that tend to cancel and average out during the spin process and it is best to allow sufficient time for this to occur. 
The Laurell model WS-400-6NPP-LITE single wafer spin coater is used in the present experiments. It is equipped with a digital process controller which allows programmable time, speed and acceleration up to 25 steps. Its rotational speed can reach up to $5000 \mathrm{rpm}$. The Laurell spin coater is suitable for rinsing or drying as well.

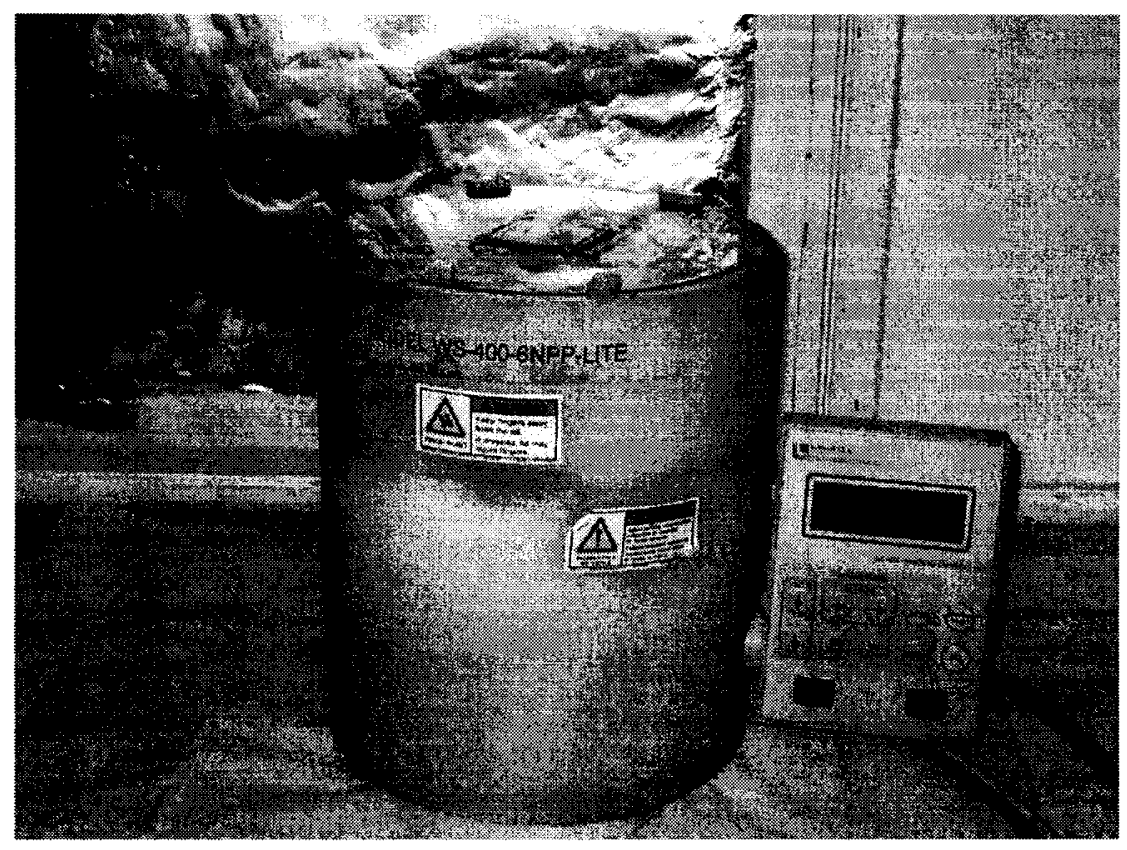

Figure 4.3: Laurell model WS-400-6NPP-Lite single wafer spin coater

\subsubsection{Photolithography}

Photolithography is the process of transferring patterns on a mask to the surface of a silicon wafer. The steps involved in this process are wafer cleaning, barrier layer formation, photoresist application, soft backing, mask alignment, exposure and development, and hard baking [4.7]. In this section, only the more important steps will be described.

There are two types of photoresist: positive and negative. For positive resists, the 
resist layer is exposed to UV light wherever the underlying material is to be removed. In these resists, exposure to the UV light changes the chemical structure of the resist so that it becomes more soluble in the developer. The exposed resist is then washed away by the developer solution, leaving windows of the bare underlying material. Negative resists behave in just the opposite manner. Exposure to the UV light causes the negative resist to become polymerized and more difficult to dissolve. Therefore, the negative resist remains on the surface wherever it is exposed, and the developer solution removes only the unexposed portions [4.7].

One of the most important steps in photolithography process is mask alignment. A mask is a square glass plate with a patterned emulsion or metal film on one side. The mask is aligned with the wafer, so that the pattern can be transferred onto the wafer surface. Except for the first one, each of the masks must be aligned to the previous pattern to ensure registration. Once the mask has been accurately aligned with the patterns on the wafer's surface, the photoresist is exposed through the patterns on the mask with a high intensity ultraviolet light. The most common exposure method is the contact printing which allows very high resolution.

In this work, the ORIEL Corp. Model 87100 Mask Aligner coupled with UV exposure system and Model 68810 Arc Lamp Power Supply are used in this experiment for the photolithography process. 


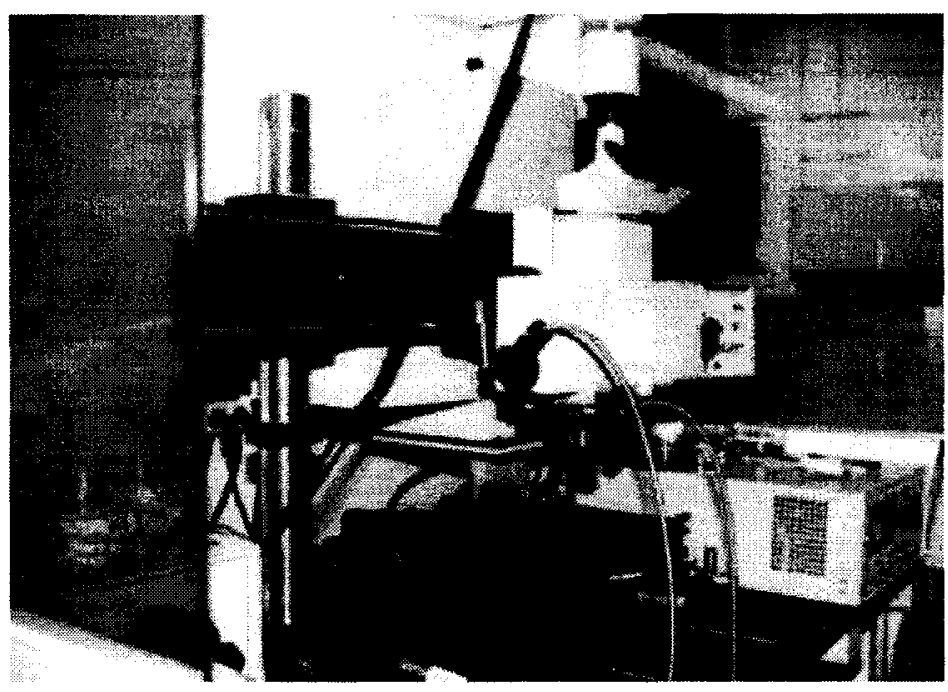

Figure 4.4: ORIEL Corp. Model 87100 Mask Aligner 


\subsection{Fabrication procedure}

As a brief description of the equipment has been given in the previous section, a detailed fabrication procedure for organic on inorganic semiconductor heterojunctions device will be described now. It will start off with a flow chart summarizing the key steps for fabricating the device. A more detailed description of different fabrication steps will then follow.

\subsubsection{Process flow chart}

Figure 4.5 shows a flow chart summarizing key steps in the fabrication of a hybrid organic on inorganic semiconductor heterojunction device used in this work.

The 2" n-type silicon wafer is first cut into smaller substrates of rectangular shape. The Si substrates are then cleaned using Acetone (ACE), Trichloroethylene (TCE) and de-ionized water in an ultrasonic bath. A wet oxidation is performed to grow a thick silicon dioxide layer on top of the substrate. Photolithography process with Mask \#1 is carried out to open the active region window on the substrate. The $\mathrm{SiO}_{2}$ is then etched away with buffered HF. Immediately after the substrate has been rinsed and dried; the organic polymer is spin-coated onto the substrate to create a uniform layer. The samples are then mounted onto the substrate holder of the vacuum system. Mask \#2 is aligned carefully with each substrate so as to create the top metal electrode with thermal evaporation of gold and chromium. After evaporation, the 
samples are removed from the vacuum chamber. Wood's alloy is applied to the backside of the silicon substrate to create the bottom electrode of the device. The fabricated device is now ready for measurements.

START<smiles>C1CCCC1</smiles>
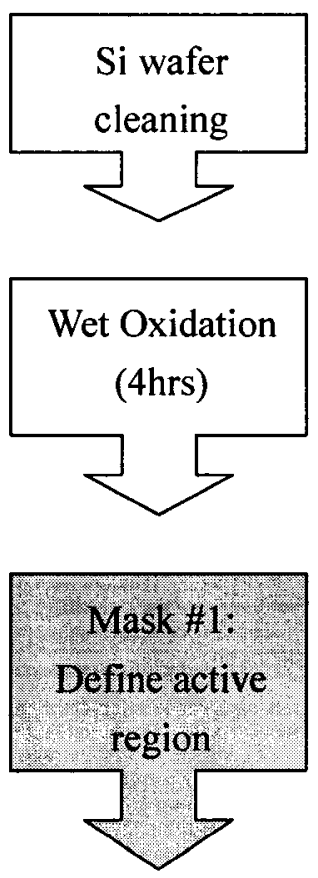

$\mathrm{SiO}_{2}$ etching

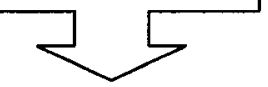

Spin-coating of organic polymer
FINISH

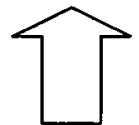

IV and CV

Measurements
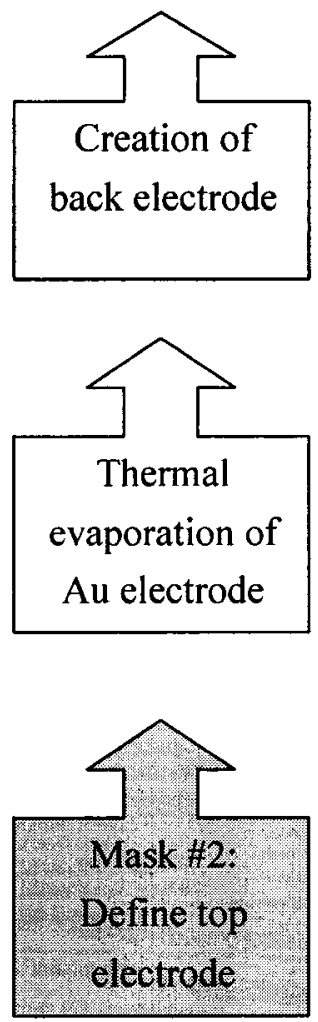

Figure 4.5: A flow chart for the fabrication of organic on inorganic semiconductor heterojunction devices. 


\section{Cross-sectional view}

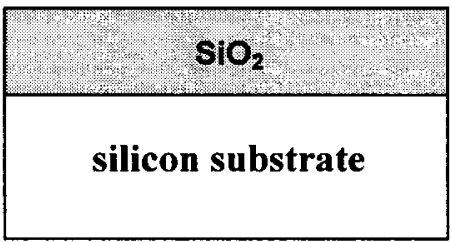

(a)
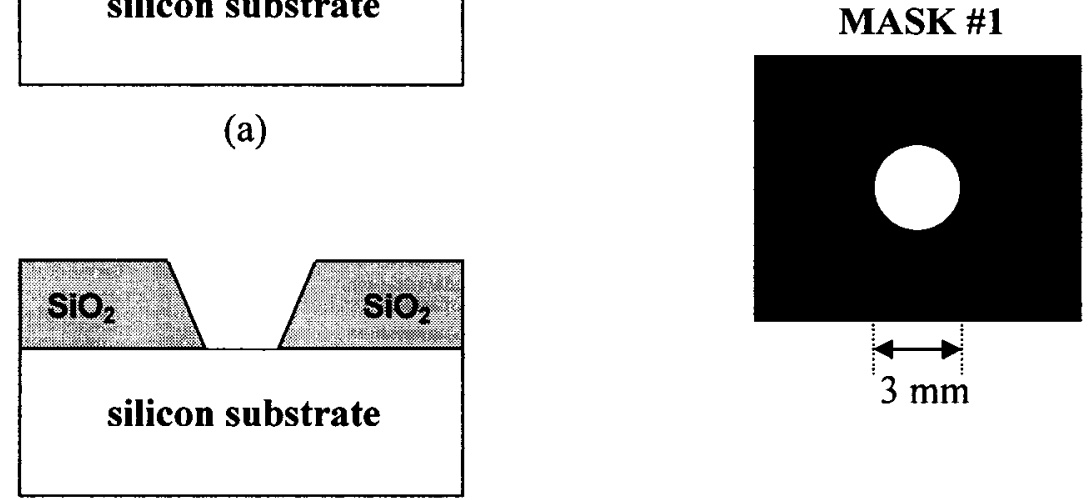

(b)

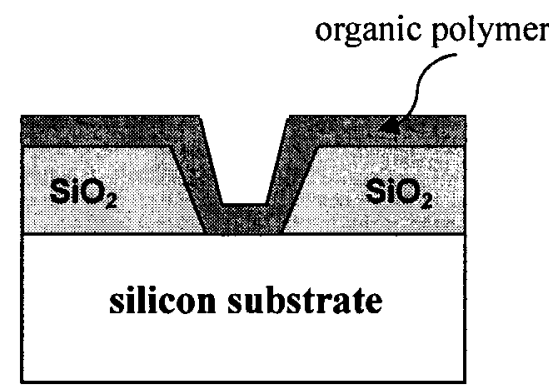

(c)

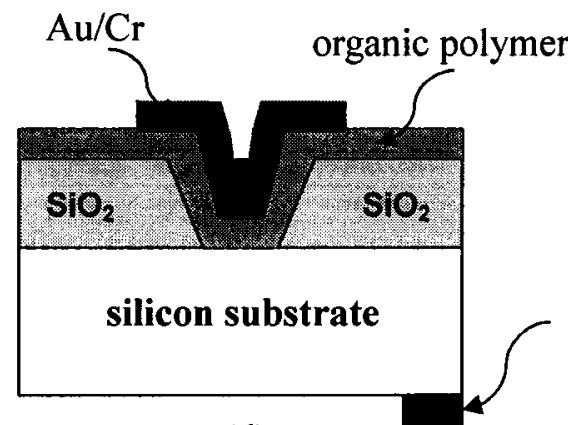

(d)

MASK \#2

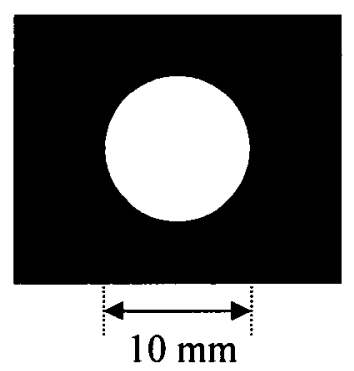

Figure 4.6: Cross-sectional views of the fabrication of organic on inorganic semiconductor heterojunction device. (a) Si substrate after oxidation; (b) active region window opening defined by Mask \#1;(c) spin-coating of polymer; and (d) Thermal evaporation of top electrode defined by Mask \#2 


\subsubsection{Description of different fabrication steps}

\subsubsection{Substrate preparation}

The 2" n-type (111) silicon wafer with resistivity around $3 \Omega-\mathrm{cm}$ is manufactured by Wacker. Figure 4.7 shows a photograph of the n-type silicon substrate used. Since the silicon wafer is being used as the inorganic substrate, cleanliness of wafer will affect the performance and yield of the devices. The wafer is first cleaned in ultrasonic bath using Acetone (ACE) for 3 minutes to remove any traces of organic materials. It is then cleaned in ultrasonic bath again but with de-ionized (DI) water for 3 minutes to wash away the ACE solvent and any other residues. The wafer is further rinsed repeatedly with DI water to remove any remaining ACE solvent and containments. Afterwards, the wafer is spin-dried and baked in air at $90{ }^{\circ} \mathrm{C}$ for 10 minutes.

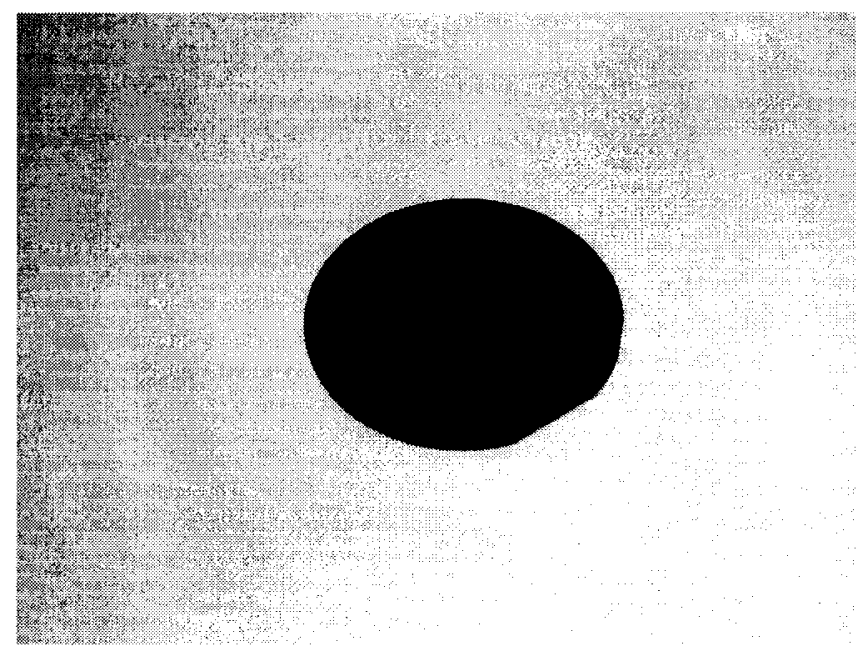

Figure 4.7: Photograph of 2" n-type (111) silicon wafer from Wacker. 
After the wafer has been cleaned, wet oxidation is performed to create a layer of silicon dioxide $\left(\mathrm{SiO}_{2}\right)$ on top of the substrate. The wet oxidation process is carried out in high temperature furnace at $1100{ }^{\circ} \mathrm{C}$ for 4 hours to grow an oxide of thickness $\sim 0.5$ $\mu \mathrm{m}$. The purpose of this oxide layer is to reduce the probability of probe needle punching through the top metal contact and the organic polymer during measurement.

The wafer is cut into smaller pieces of rectangular shape for easier handling in the later steps.

\subsubsection{Photolithography using Mask \#1}

Mask \#1 is a rectangular opaque plate with circular opening of diameter $3 \mathrm{~mm}$ to define the active region of the device as shown in Figure 4.6(b). The following photolithography process is carried out to open the active region window.

1) Spin Shipley AZ 1827 photoresist onto the samples at 3000 rpm for 20 seconds.

2) Pre-bake in air at $90{ }^{\circ} \mathrm{C}$ for 10 minutes.

3) Align Mask \#1 with the sample and expose the photoresist under UV light at 300 Watts for 300 seconds.

4) Develop the samples using diluted Shipley Microposit Developer.

5) Post-bake the samples at $110^{\circ} \mathrm{C}$ for 10 minutes.

6) Cool the samples to room temperature. 


\subsubsection{Silicon dioxide etching}

After the photolithography process, the following silicon dioxide etching is performed.

1) The sample is immersed into the buffered HF solution to etch away the thick $\mathrm{SiO}_{2}$ to open the active region window. The etching process may take more than 15 minutes.

2) Rinse in DI water to remove any etched residue.

3) Remove photoresist with acetone.

4) Clean the sample thoroughly with DI water.

5) Bake-dry the sample in air at $90{ }^{\circ} \mathrm{C}$ for 5 minutes.

\subsubsection{Organic polymer Spin-coating}

A few different organic polymers (MEH-PPV, P3HT and PEDOT) are used in this work. Figure 4.8 displays the various polymers in their bottles while Figure 4.9 shows photographs of MEH-PPV POSS and P3HT polymers in their solid form. Detail of the polymers characteristics in this work will be given in the next chapter. The following procedure describes the general spin-coating of the polymers onto the substrate immediately after the active region window has been opened.

1) Apply a few drops of the organic polymer solution to the sample. 
2) Spin the sample at $2000 \mathrm{rpm}$ for 20 seconds.

A cross-sectional view of the sample now is shown in Figure 4.6(c). The samples have to be loaded into vacuum chamber as soon as possible to reduce any interaction of the organic polymer with the air as this may cause device performance degradation.

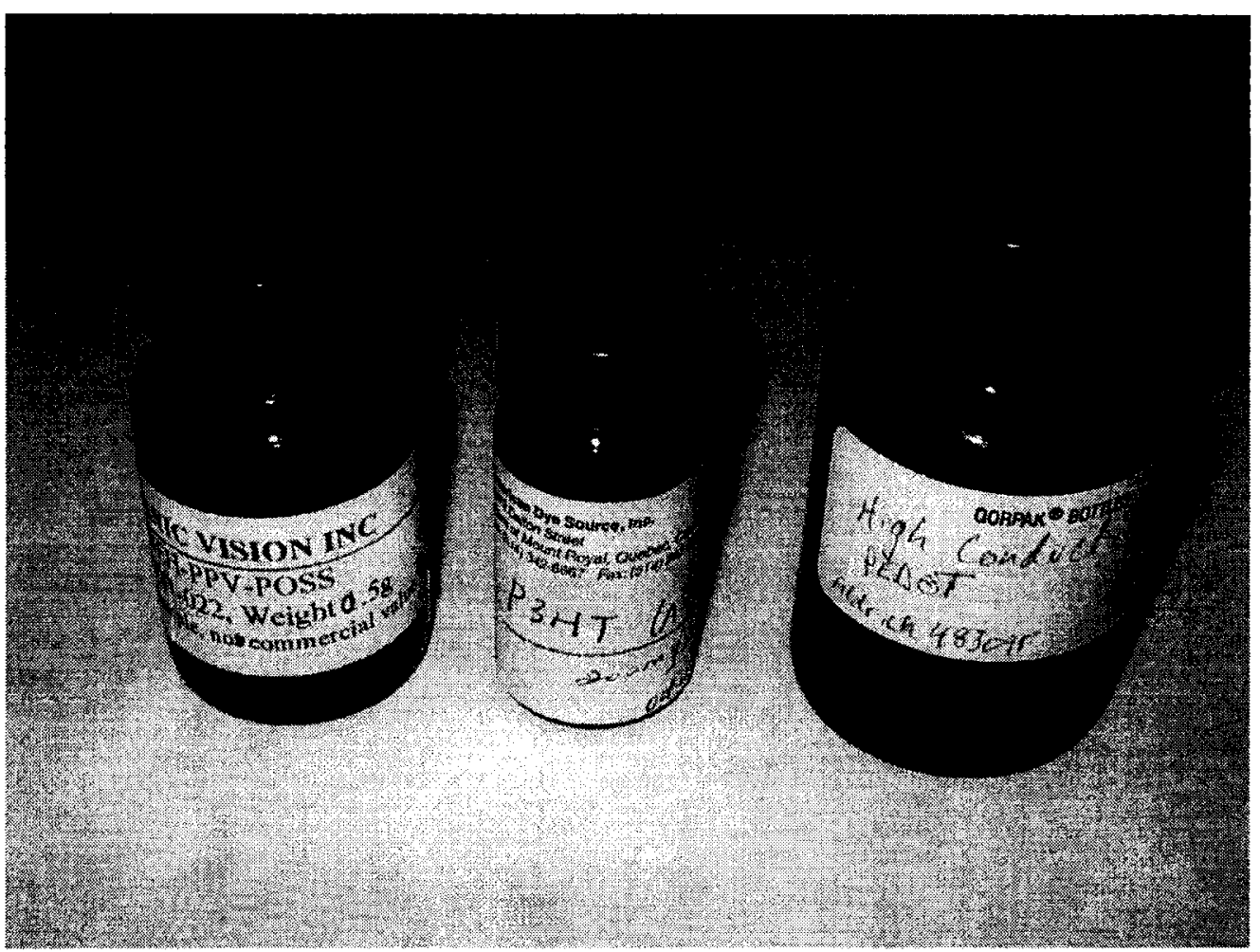

Figure 4.8: Photograph of the various polymers in their bottle.

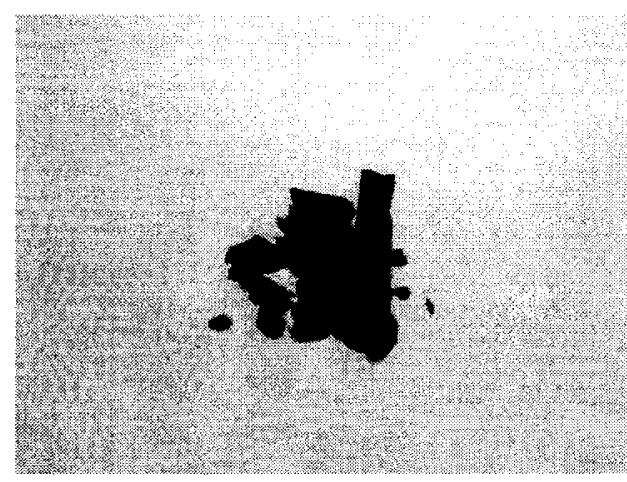

(a)

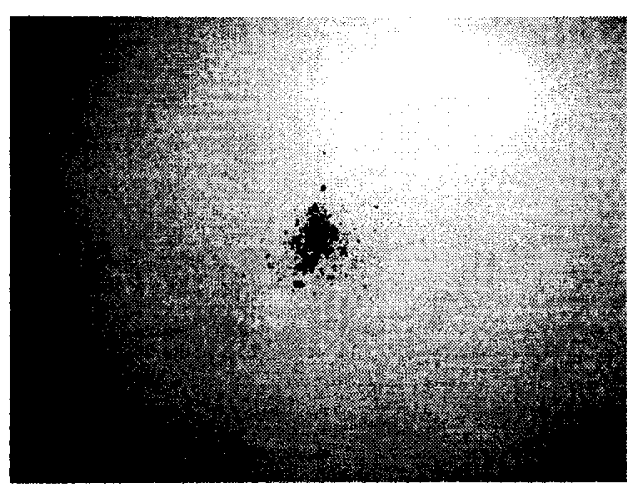

(b)

Figure 4.9: Photographs of (a) MEH-PPV POSS and (b) P3HT 


\subsubsection{Thermal evaporation of gold using Mask \#2}

The next step in the fabrication is to evaporate a layer of gold and chromium $(\mathrm{Au} / \mathrm{Cr})$ as the top electrode of the device. The following describes the thermal evaporation process being carried out.

1) Mount the sample facing downward on the substrate holder.

2) Align the metal Mask \#2 with the sample and fix its position with a clip.

3) Load the substrate holder back into the chamber.

4) Cut a small piece of high purity gold and place it into the filament boat of thermal evaporation system.

5) When the vacuum system reaches a pressure less than $10^{-5}$ Torr, make sure the shutter is in closed position and turn on the evaporation power supply.

6) Slowly increase the voltage until gold piece starting to melt.

7) Open the shutter to allow deposition, close the shutter and reduce the voltage slowly after desired thickness has been achieved.

8) Wait for 10 minutes before removing the sample from the vacuum system.

9) Apply some Wood's alloy to the backside of the substrate using a soldering iron tip to serve as the bottom electrode of the device.

The whole fabrication process is complete and the fabricated organic on inorganic semiconductor heterojunction device is ready for measurement. 


\subsection{Conclusions}

In this chapter, the fabrication processes of hybrid organic on inorganic semiconductor heterojunction devices have been described. Two methods are available for depositing the organic layer: vacuum deposition and spin-coating. Spin-coating method was used in the present work as it is fast and economical. However, devices with the spin-coated organic layer may have a slightly lower performance compared to one with vacuum deposited organic layer as the spin-coated organic layer is usually less uniform and possibly has more defects.

Experimental setup used to fabricate the organic on inorganic semiconductor heterojunction devices was given. Principles of thermal vacuum evaporation system, spin-coat processor and the photolithography process were briefly explained.

The fabrication procedure for the devices was summarized in a process flow chart with reference to the cross-sectional views of the devices after each step. Detailed descriptions of each step such as substrate preparation, photolithography, organic polymer spin-coating and thermal evaporation of metal contact were given.

In conclusion, a detailed fabrication process of hybrid organic on inorganic semiconductor heterojunction devices was covered in this chapter. 


\subsection{References}

[4.1] Hoppe, H and Sariciftci, N. (2004) J. Mater. Res., Vol. 19, No. 7, 1924-1945

[4.2] Thelakkat, M. Schmitz, C. Schmidt, HW. (2004) Adv Mater. 14(8) 5777

[4.3] Schroeder, R. and Ullrich, B. (2002) Mat. Res. Soc. Symp. Proc. Vol. 708

pp.29-32

[4.4] Richard C. Jaeger, (2002) “Modular Series on Solid State Devices, Volume VIntroduction to Microelectronic Fabrication," $2^{\text {nd }}$ ed. Prentice-Hall, Ch. 6

[4.5] Chopra, KL. and Kaur, I. (1983) "Thin Film Device Applications", Plenum Press, New York, Ch.1 pp. 14-18

[4.6] Wolf, S. and Tauber, RN. (2000), "Silicon Processing for the VLSI Era Volume

1 - Process Technology," Lattice Press, Ch. 12

[4.7] Georgia Institute of Technology Virtual Cleanroom

http://www.ece.gatech.edu/research/labs/vc/theory/photolith.html 


\section{Measurement Setup and}

\section{Experimental Results}

\subsection{Overview}

In this work, the research effort is primary directed towards enhancing the understanding of governing principles in these semiconducting organic materials to provide information necessary for improving the performance of hybrid organic on inorganic semiconductor heterojunction devices. In order to study their electrical characteristics, the organic on inorganic semiconductor heterojunction devices have been fabricated using the experimental setup described in Chapter 4. Several semiconducting polymers have been selected and were studied in this work.

Various means of measurement, such as current-voltage and capacitance-voltage measurements, have been performed to characterize the electrical properties of the devices. Measurement setup of the equipment for the characterizations will be described first in this chapter followed by a presentation of the experimental results obtained for the various organic on inorganic semiconductor heterojunction devices. 


\subsection{Measurement Setup}

The two most basic techniques for electrical characterization of semiconductor devices are the current-voltage (I-V) and capacitance-voltage (C-V) measurement respectively. I-V measurement determines electrical output performance of devices, such as threshold voltage, junction ideality factor and series resistance. Several parameters of semiconductor materials and structures can be determined by measuring $\mathrm{C}-\mathrm{V}$ characteristics. These parameters include doping profile, interface traps, volume traps or mobile ionic charges. Some fundamental knowledge of measurement theory on traditional semiconductor devices will be covered in this section. Description of the measurement setup will be included as well.

\subsubsection{Current-Voltage Measurements}

The I-V characteristics of the fabricated organic on inorganic semiconductor heterojunction devices were measured to determine the device performance using a Hewlett-Packard HP4145A semiconductor parameter analyzer. A personal computer with a GPIB interface card is connected to the parameter analyzer through an IEEE-488 standard cable with 24-pin plug and receptacle connectors at each end to control the device measurements and data acquisition. The devices to be tested were mounted onto a holder stage with two probing needles connected to the top and bottom contacts of the devices respectively. All the devices were characterized in 
atmospheric ambient at room temperature under dark condition unless specified otherwise. A schematic diagram of the measurement setup for the I-V measurements is shown in Figure 5.1.

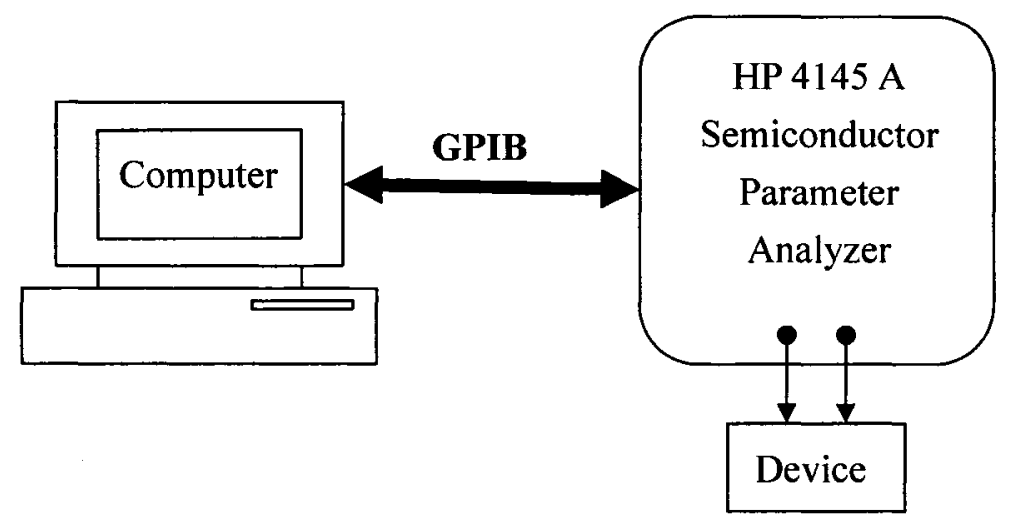

Figure 5.1: A schematic diagram of device I-V measurement setup

In Chapter 3, it has been explained that in regard to transport at inorganic semiconductor - conjugated polymer interfaces, nearly free-electron thermionic emission theory is generally assumed. For a typical inorganic semiconductor diode, the I-V relation qualitatively follows the phenomenological diode equation:

$$
I=I_{o}\left[\exp \left(\frac{e V_{a}}{k T}\right)-1\right]
$$

where $I_{0}$ is saturation current; $V_{a}$ is the applied voltage; $k$ is the Boltzmann's constant $\left(8.62 \times 10^{-5} \mathrm{eV} / \mathrm{K}\right)$; and $\mathrm{T}$ is the temperature. This is also known as the ideal-diode equation, gives a good description of the current-voltage characteristics of the 
junction. The ideality factor, $\mathrm{n}$, which is a measure of how close the forward current mechanism is to ideal, is obtained by fitting the experimental $\ln (\mathrm{I})$ versus $V_{a}$ characteristic by a straight line given by the following equation:

$$
n=\frac{\kappa T}{e}\left(\frac{d \ln I}{d V_{a}}\right)^{-1}
$$

or in a form similar to $y=A x+B$ when plotted on the semilog axis

$$
\ln (I)=\frac{e V_{A}}{n \kappa T}+\ln \left(I_{o}\right)
$$

The slope of the line and the $V=0$ intercept yield the value of $n$ and $I_{0}$ respectively. Usually, the I-V relationship for a non-ideal $\mathrm{p}-\mathrm{n}$ junction exhibits three regions of different behavior as illustrated in Figure 5.2.

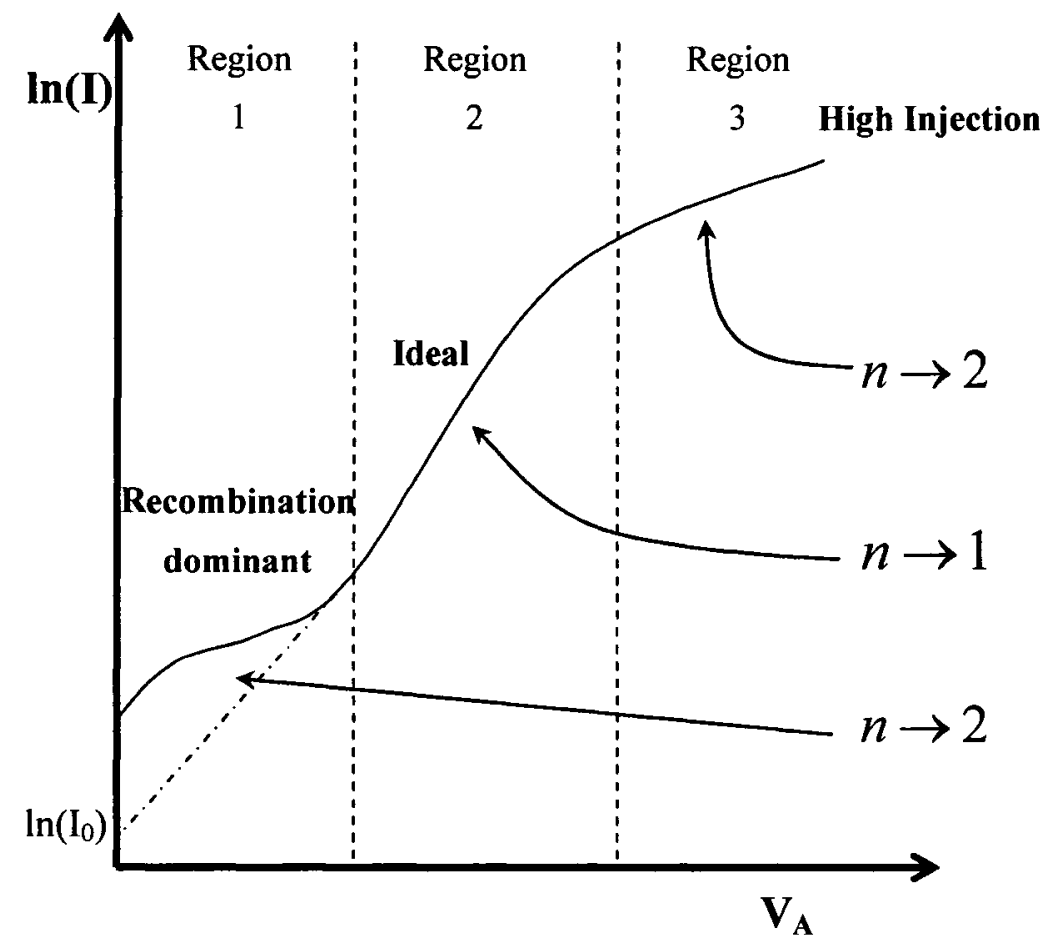

Figure 5.2: Forward biased deviations from an ideal p-n junction 
For an ideal diode, it is assumed that electrons and holes injected across the depletion region barrier are not able to recombine with each other. Only when they enter the neutral regions, they are able to recombine with the majority carriers. However, in a real diode, a number of sources may lead to bandgap states, for example defects such as vacancies, interstitials or chemical impurities. As electrons and holes enter the depletion region, one possible way they can cross the region without overcoming the potential barrier is to recombine with each other. This leads to an additional flow of charged particle. This current is called generation-recombination current, and must be added to the total current [5.1]. At very low levels of current, the recombination current component dominates in the depletion region and usually $\mathrm{n}$ approaches 2 in many silicon devices. However, as the applied bias increases, the diffusion current starts to dominate and thus ideality factor $\mathrm{n}$ approaches 1 . As the forward bias is increased even more, the injection level increases and eventually the injected minority carrier density becomes comparable to the majority carrier density. When this happens, an increasingly large fraction of external bias drops across the undepleted region. The diode current will then stop increasing exponentially with the applied voltage, but still tend to saturate as shown in region 3 of Figure 5.2. The ideality factor $\mathrm{n}$ tends to deviate from 1 in this region as well.

In the derivation of the ideal diode equation, it was assumed that the electric field 
in the bulk $\mathrm{n}$ and p-regions was approximately zero for the minority carriers and that the no voltage drop existed across the ohmic contacts [5.2]. At high current injection, the bulk resistance can produce a significant voltage drop and the applied voltage is larger than the voltage drop across the depletion region. The ohmic contacts can behave as a small resistor, adding to the voltage drop. Usually, these two effects are combined into a resistor $R_{S}$, series resistance. One method used to measure $R_{S}$ is to plot the voltage drop from ideal $(\Delta \mathrm{V})$ versus current on a linear scale and $\mathrm{R}_{\mathrm{S}}$ can be determined from the slope of the best-fit line.

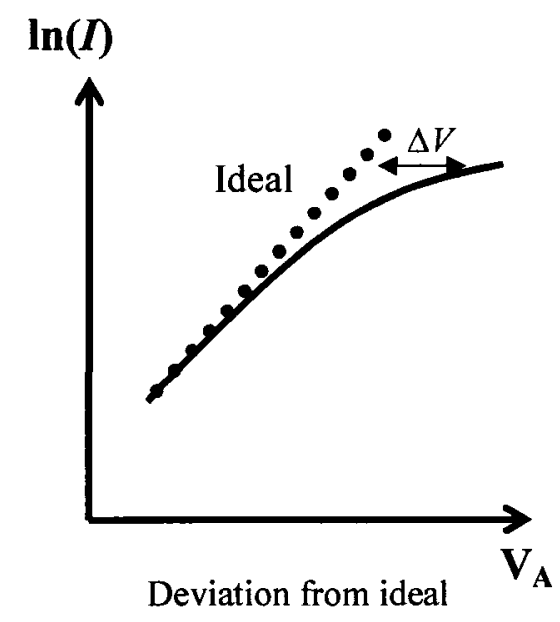

(a)

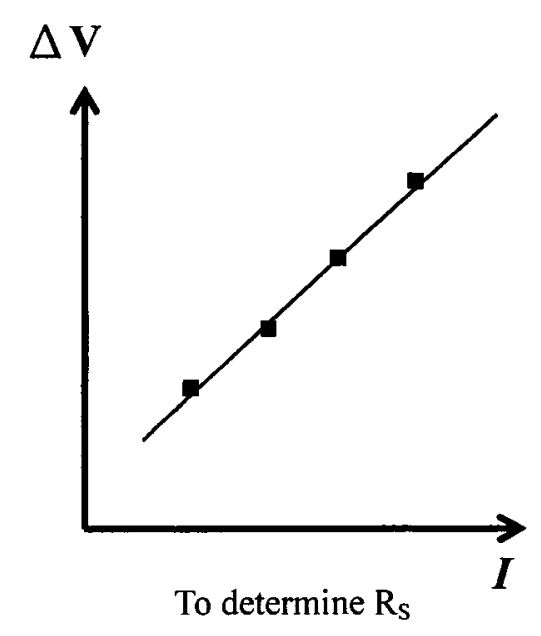

(b)

Figure 5.3: Two steps to determine $\mathrm{R}_{\mathrm{S}}$ (a) obtain deviation of $\mathrm{V}$ at $I$, and (b) plot $\Delta$ V vs. $I[5.2]$

Another important parameter in the I-V measurement of the organic on inorganic semiconductor heterojunction is the reverse leakage current density. The 
value of the reverse leakage current density is calculated by measuring the current density at a fixed reverse bias voltage such as $-1 \mathrm{~V}$ or $-2 \mathrm{~V}$.

\subsubsection{Capacitance-Voltage Measurements}

The measurement setup for determining the capacitance-voltage (C-V) characteristic is very similar to that of the I-V measurements. The C-V measurements were carried out using a Hewlett-Packard multi-frequency HP 4274A LCR meter, which is controlled by a personal computer as shown in Figure 5.4. The dc bias voltage is monitored using an HP 3478A multimeter. In the $\mathrm{C}-\mathrm{V}$ measurements, the bias voltage was varied from -2.5 to $0 \mathrm{~V}$ at room temperature with a frequency of 100 $\mathrm{kHz}$.

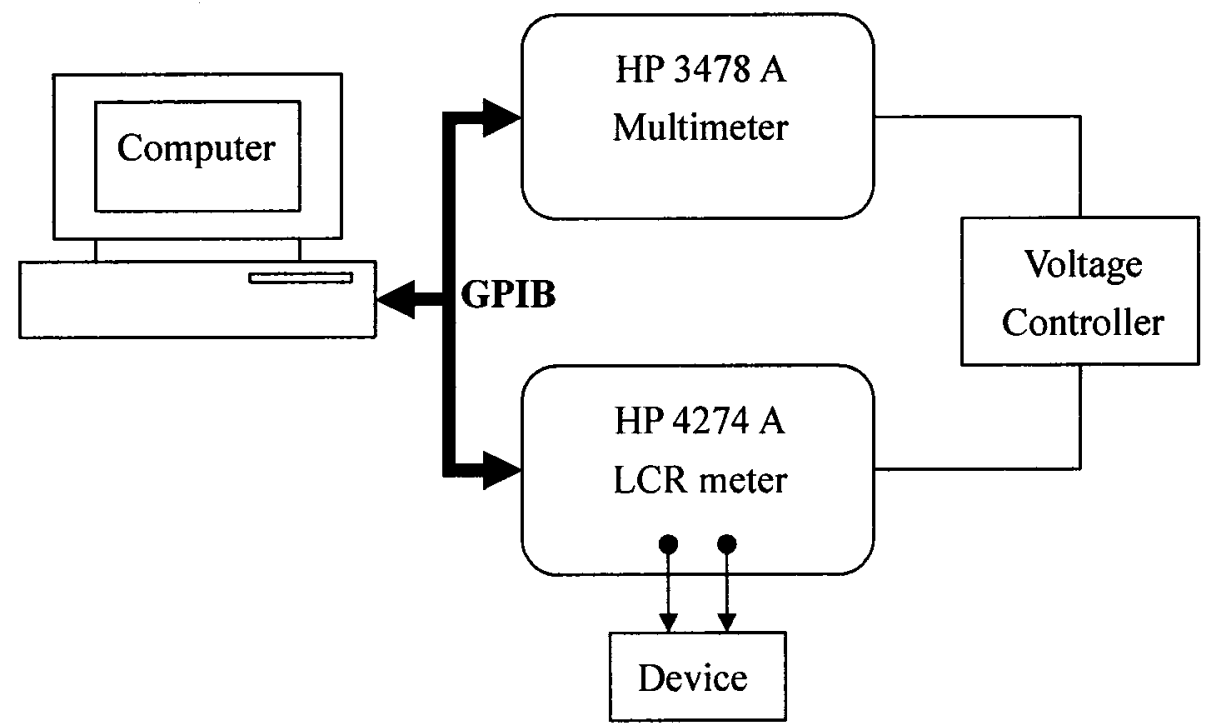

Figure 5.4: Schematic diagram of the C-V measurement setup. 
Sometimes, the electrical behavior of a p-n heterojunction may be related to the trapping levels in the bulk semiconductors or to states at the interface. Such levels may result from crystal imperfections such as lattice defects, impurities, non-stoichiometry, and microcrystalline grain boundaries [5.3]. Differential C-V measurements may be used to obtain qualitative information on these imperfections. The method of measuring free carrier (or doping) concentration profiles in semiconductors by $\mathrm{C}-\mathrm{V}$ relies on modulating the depletion region in a diode or $\mathrm{p}-\mathrm{n}$ junction by an applied voltage [5.4]. It requires a junction in which a reverse-biased space-charge region can be created. Superposition of a small ac voltage onto the dc bias allows the measurement of a capacitance of a thin region of doped material underneath a contact with well-defined geometry (i.e., in which the area is precisely known). Scanning the reverse dc bias of the doped material provides a measure of the free carrier concentration versus depth. In simple structures such as Schottky barriers or $\mathrm{p}-\mathrm{n}$ junctions the extraction of the doping profile is relatively simple. If all the dopant atoms are electrically active, then this information is obtained by analyzing the $\mathrm{C}-\mathrm{V}$ characteristics, where the depletion depth is proportional to the area divided by the capacitance.

The capacitance of a one-sided junction $\left(p^{+} n\right)$ can be given by the following equation: 


$$
\frac{1}{C^{2}}=\frac{2\left(V_{b i}-V_{A}\right)}{q \varepsilon_{s} N_{D}}
$$

where $\mathrm{V}_{\mathrm{A}}$ is applied bias voltages and $\varepsilon_{\mathrm{s}}, \mathrm{N}_{\mathrm{D}}$ are permittivity and doping concentration for the lightly doped region (n-Si region in this case) respectively. The $\mathrm{V}_{\mathrm{bi}}$ can be determined by extrapolating the curve to the point where $1 / \mathrm{C}^{2}=0$ and the slope of the curve is inversely proportional to the doping concentration of the lightly doped region in the junction.

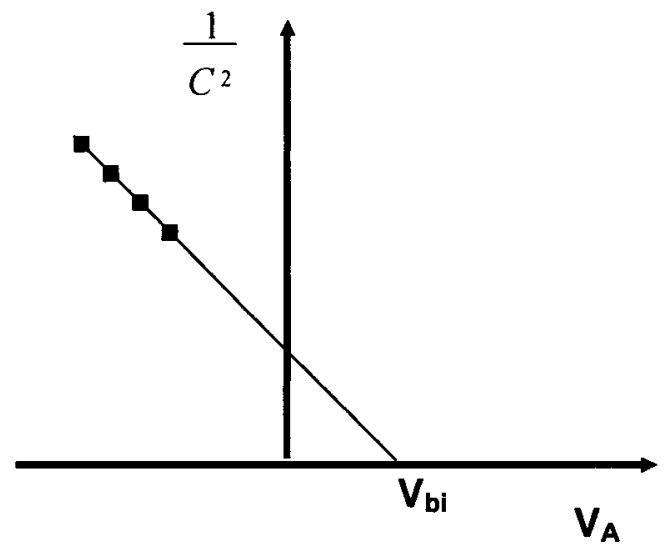

Figure 5.5: $\left(1 / \mathrm{C}^{2}\right)$ versus $\mathrm{V}_{\mathrm{A}}$ for a $\mathrm{p}^{+} \mathrm{n}$ junction.

A junction can be reversed biased only before the breakdown. This condition imposes a profile depth limit on the sample. For heavily doped junction, as are generally encountered for diffused or ion-implanted layers, the depth at breakdown is very small. For heterojunction cells, the $\mathrm{C}-\mathrm{V}$ plot usually has two separate regions: one from low reverse bias to forward bias (shallow region, near the depletion region), and the other from low reverse bias to large reverse bias (deep region). This is because the charging of interface states, which alters the junction barrier, is affected 
by the applied voltage. This charging effect does not occur in a homojunction in which usually less interface states exist. Thus, in order to obtain $\mathrm{V}_{\mathrm{bi}}$ without the interference from the interface states charging effect, the large reverse bias region is considered. The same is applied to the calculation of the slope. 


\subsection{Experimental Results}

As stated in Chapter 1, the objective of this work is to study the hybrid organic on inorganic semiconductor heterojunction devices. Several devices with different organic polymer on silicon substrate were fabricated using the procedure outlined in the pervious chapter. $\mathrm{I}-\mathrm{V}$ and $\mathrm{C}-\mathrm{V}$ measurements were performed on the devices in order to study their performances. In the following sub-sections, the experimental results obtained for selected working devices will be presented according to the organic polymer used.

\subsubsection{MEH-PPV POSS}

Poly(para-phenylene vinylene) or PPV is the simplest polymer comprising of alternating benzene and vinylene units. The material is highly fluorescent and is bright yellow in color. It is one of the most widely studied semiconducting organic materials used mainly in light emitting applications. However, conjugated polymers in general have tendencies to aggregate or stack as a consequence of their extensive $\pi$-delocalization so that they are usually insoluble in most solvents [5.5]. Therefore, they are often coated in soluble unconjugated precursors form with subsequent thermal conversion to form soluble polymers. The poly(2-methoxy-5(2-ethylhexyloxy) -1,4-phenylenevinylene) (MEH-PPV), structure as shown in Figure 5.6, was first introduced by the group at the University of California at Santa Barbara 
[5.6]. Its enhanced solubility can be attributed to the branched nature of its side-chain.

It is known that hybrid organic-inorganic polymers containing segments of polyhedral oligomeric silsesquioxanes (POSS) exhibit a number of potentially useful properties, including high thermal stability in air and good adhesion to a number of substrates [5.7]. MEH-PPV POSS used in this work was prepared by dissolving 20 $\mathrm{mg}$ of the organic polymer in $8 \mathrm{~g}$ of chloroform. The inorganic semiconductor counterpart is n-type silicon substrate.

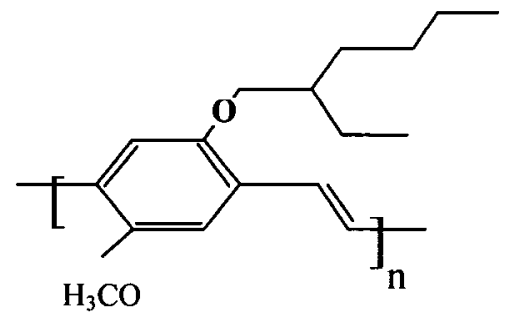

(a)

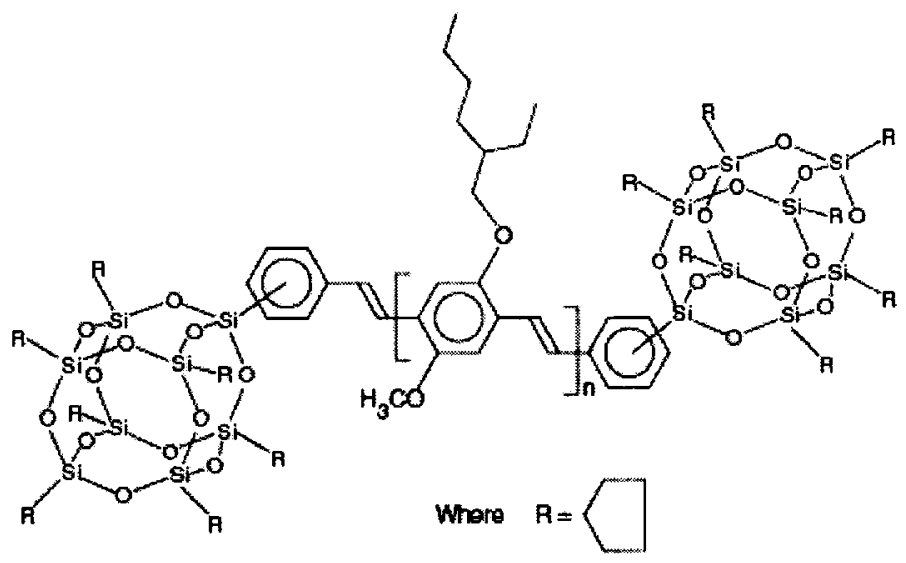

(b)

Figure 5.6: Molecular structure of a) MEH-PPV and b) MEH-PPV POSS [5.7] 


\subsubsection{Current-Voltage Characteristics}

In the beginning of this work, several MEH-PPV POSS on n-Si heterojunction devices were fabricated using a procedure similar to that described in Chapter 4. However, their structures and size of active regions were slightly different. Figure 5.7 shows a picture of the devices fabricated. I-V measurements were carried out on the devices and a summary of some of the key parameters are presented in Table 5.1 and 5.2 below.

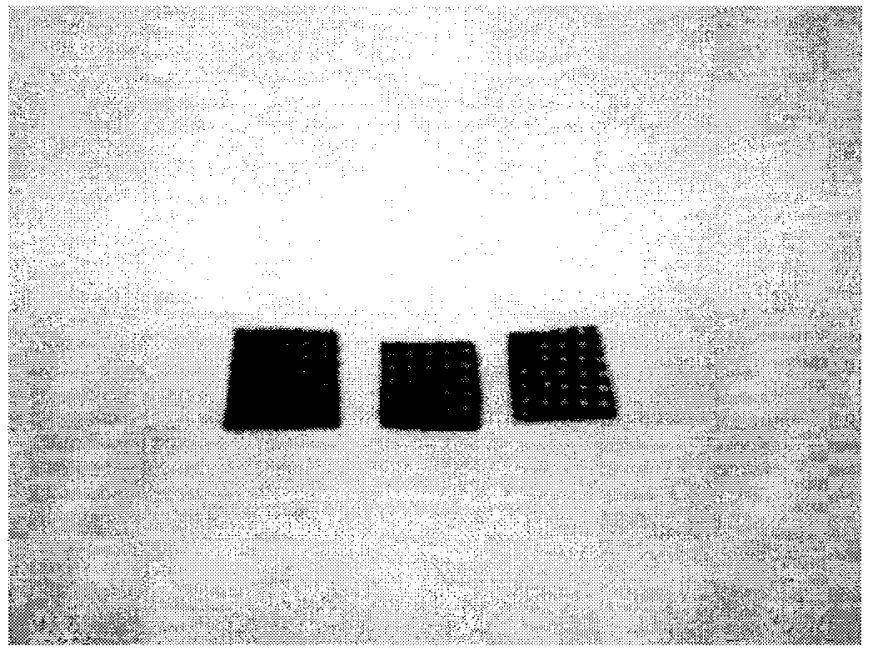

Figure 5.7: A photograph of MEH-PPV POSS on n-Si heterojunction devices. 
Forward Current vs Voltage Characteristics for 3 MEH-PPV POSS on n-Si Heterojunctions (with Al contact)

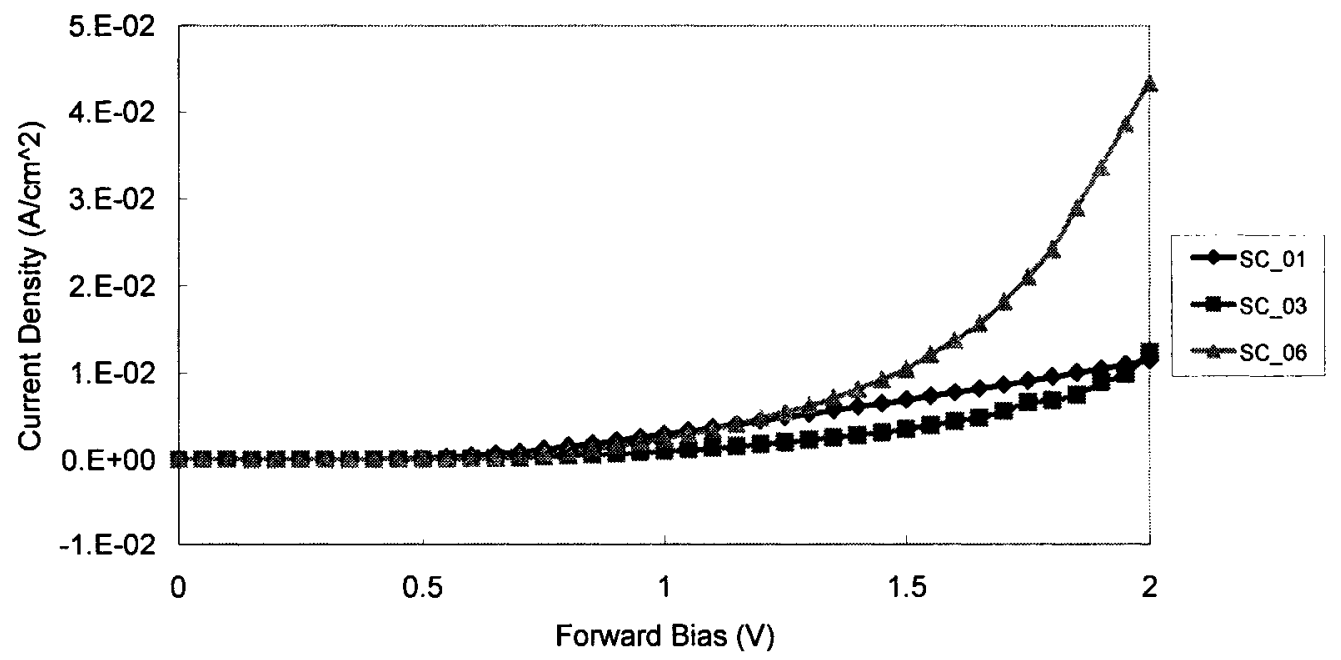

Figure 5.8: J-V characteristics for MEH-PPV POSS on n-Si heterojunctions with Al contact in forward bias

Forward In (I) vs Voltage Characteristics for 3 MEH-PPV POSS on n-Si Heterojunctions (with Al contact)

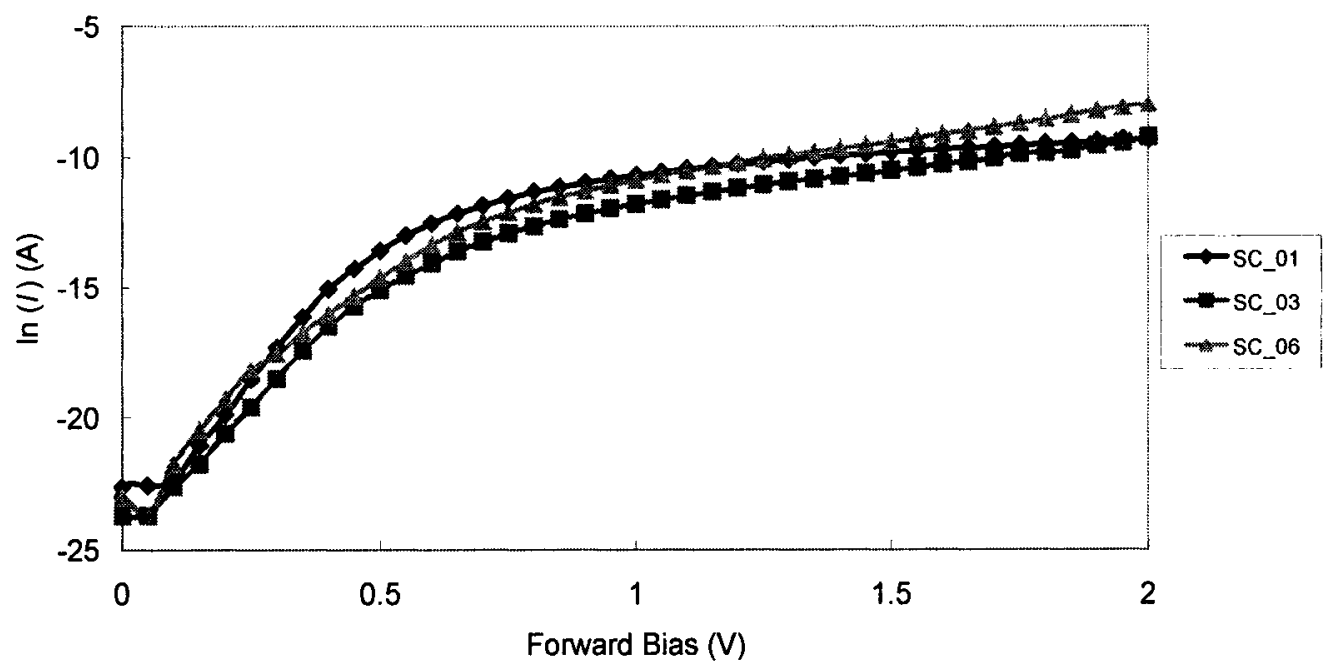

Figure 5.9: $\ln (I)-\mathrm{V}$ characteristics for MEH-PPV POSS on n-Si heterojunctions with Al contact in forward bias 
Table 5.1: Summary of the J-V characteristics for MEH-PPV POSS on n-Si heterojunctions with Al contact

\begin{tabular}{|c|c|c|c|c|}
\hline Organic polymer & \multicolumn{4}{|c|}{ MEH-PPV POSS } \\
\hline $\begin{array}{c}\text { Inorganic } \\
\text { semiconductor }\end{array}$ & \multicolumn{4}{|c|}{ n-type silicon } \\
\hline Anode Contact & \multicolumn{4}{|c|}{ aluminium } \\
\hline Device Area & \multicolumn{4}{|c|}{$7.9 * 10^{-3} \mathrm{~cm}^{2}$} \\
\hline Sample \# & $S C \_01$ & SC_03 & SC_06 & Average \\
\hline $\begin{array}{c}\text { Rectification Ratio } \\
(|\mathbf{1 V}|)\end{array}$ & $1.5 \times 10^{2}$ & $1.1 \times 10$ & $3.0 \times 10$ & $6.4 \times 10$ \\
\hline $\begin{array}{c}\text { Rectification Ratio } \\
(|\mathbf{2 V}|)\end{array}$ & $1.3 \times 10^{3}$ & $1.4 \times 10$ & $1.1 \times 10^{2}$ & $4.7 \times 10^{2}$ \\
\hline $\begin{array}{l}\text { Saturation Current } \\
\text { Density } J_{0}\left(\mathrm{nA} / \mathrm{cm}^{2}\right)\end{array}$ & 22 & 29 & 18 & 23 \\
\hline $\begin{array}{c}\text { Leakage Current } \\
\text { Density }\left(\mu \mathrm{A} / \mathrm{cm}^{2}\right)\end{array}$ & 1.9 & 8.9 & 81 & 31 \\
\hline $\begin{array}{c}\text { Series Resistance } \\
(\Omega)\end{array}$ & $1.4 \times 10^{7}$ & $5.6 \times 10^{6}$ & $1.5 \times 10^{6}$ & $7.0 \times 10^{6}$ \\
\hline Ideality factor (n) & 1.6 & 1.8 & 1.6 & 1.7 \\
\hline
\end{tabular}

Forward J-V characteristics of the devices fabricated are shown in Figure 5.8.

These characteristics showed a rectifying behaviour of the heterojunctions indicating the presence of a potential barrier. In Table 5.1, the rectification ratio $R_{R}$ was evaluated at applied voltages of $1 \mathrm{~V}$ and $2 \mathrm{~V}$ respectively. It was observed that the average value of $R_{R}$ for MEH-PPV POSS on $n-S i$ heterojunctions with aluminium anode, are 64 (at $1 \mathrm{~V}$ ) and $4.7 \times 10^{2}$ (at $2 \mathrm{~V}$ ) respectively. The average magnitude for the saturation current density $\mathrm{J}_{0}$ and reverse leakage current density were determined to be $23 \mathrm{nA} / \mathrm{cm}^{2}$ and $31 \mu \mathrm{A} / \mathrm{cm}^{2}$. The reverse leakage current density is relatively small except for device SC_06 which has a leakage current density almost 10 times larger 
than the other two similar devices. The average series resistance $R_{S}$ was calculated to be around $7.0 \mathrm{M} \Omega$ as well. Figure 5.9 showed the current log plot of the heterojunctions in forward bias. It is apparent that the log plot resembles closely to that of a non-ideal inorganic diode. The $\mathrm{n}$ factors listed in Table 5.1 are calculated using the current measured from region 2 of the heterojunction. The fabricated heterojunctions have $\mathrm{n}$ greater than 2 in region 1 , indicating that recombination current is dominant at small forward-bias voltage. For region $2, \mathrm{n}$ factor is around 1.7, indicating a recombination current component still exists. At high injection voltage, $n$ factor becomes extremely large due to the series resistance effect. Next, the J-V characteristics for MEH-PPV POSS on $\mathrm{n}-\mathrm{Si}$ heterojunctions with Mg-Ag contact instead of Al will be presented.

Fonward Current vs Voltage Characteristics for 3 MEH-PPV POSS on n-Si Heterojunctions (with MG-Ag contact)

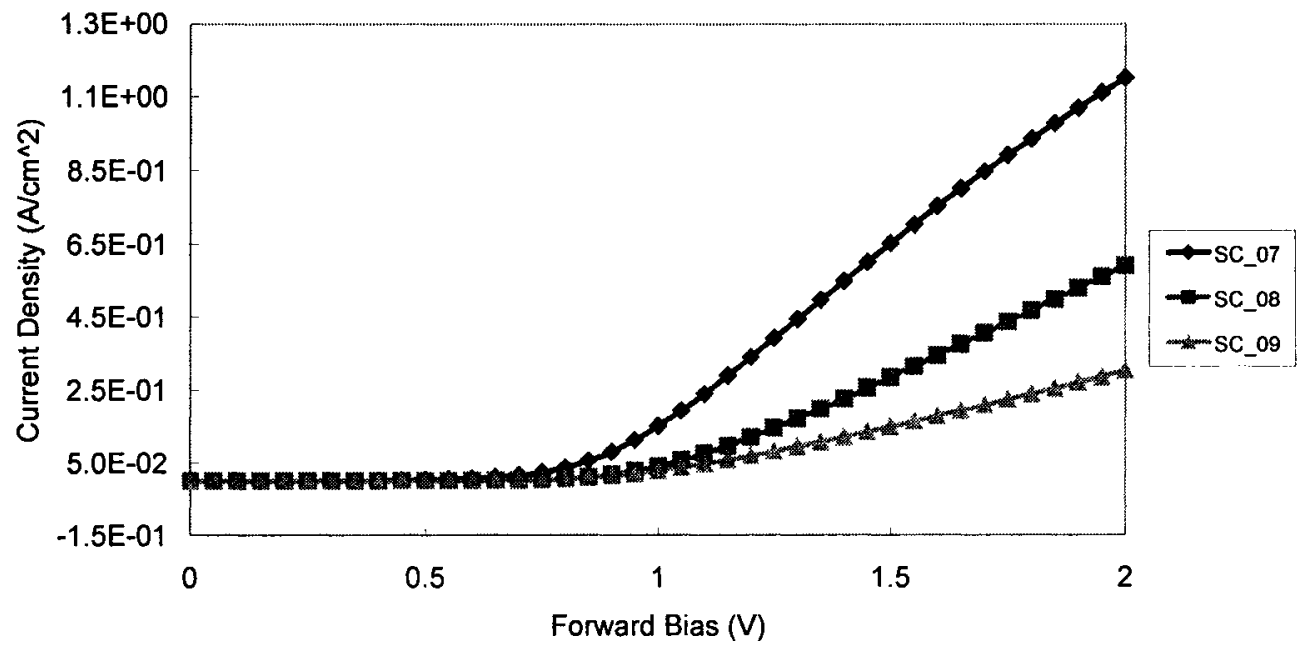

Figure 5.10: J-V characteristics for MEH-PPV POSS on n-Si heterojunctions with $\mathrm{Mg}-\mathrm{Ag}$ contact in forward bias 
Forward In (I) vs Voltage Characteristics for 3 MEH-PPV POSS on n-Si Heterojunctions (with $\mathrm{Mg}$-Ag contact)

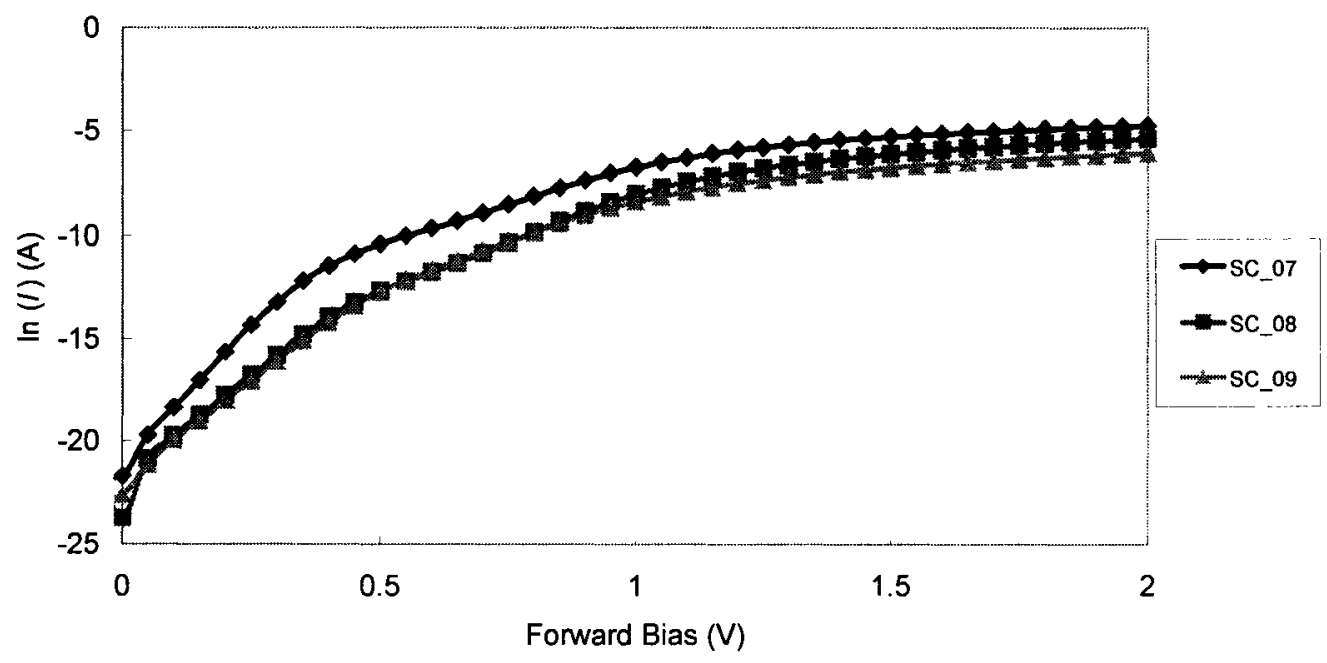

Figure 5.11: $\ln (I)-\mathrm{V}$ characteristics for MEH-PPV POSS on n-Si heterojunctions with $\mathrm{Mg}-\mathrm{Ag}$ contact in forward bias

Table 5.2: Summary of the J-V characteristics for MEH-PPV POSS on n-Si heterojunctions with $\mathrm{Mg}$-Ag contact

\begin{tabular}{|c|c|c|c|c|}
\hline Organic polymer & \multicolumn{4}{|c|}{ MEH-PPV POSS } \\
\hline $\begin{array}{c}\text { Inorganic } \\
\text { semiconductor }\end{array}$ & \multicolumn{4}{|c|}{ n-type silicon } \\
\hline Anode Contact & \multicolumn{4}{|c|}{ Mg-Ag } \\
\hline Device Area & \multicolumn{4}{|c|}{$7.9 * 10^{-3} \mathrm{~m}^{2}$} \\
\hline Sample \# & $S C \_07$ & $S C_{-} 08$ & SC_09 & Average \\
\hline $\begin{array}{c}\text { Rectification Ratio } \\
(|1 \mathrm{~V}|)\end{array}$ & $8.1 \times 10$ & $3.1 \times 10$ & $2.4 \times 10$ & $4.5 \times 10$ \\
\hline $\begin{array}{c}\text { Rectification Ratio } \\
(|\mathbf{2 V}|)\end{array}$ & $2.0 \times 10^{2}$ & $1.6 \times 10^{2}$ & $8.0 \times 10$ & $1.5 \times 10^{2}$ \\
\hline $\begin{array}{l}\text { Saturation Current } \\
\text { Density } J_{0}\left(\mathrm{nA} / \mathrm{cm}^{2}\right)\end{array}$ & 97 & 36 & 32 & 55 \\
\hline $\begin{array}{c}\text { Leakage Current } \\
\text { Density }\left(\mu \mathrm{A} / \mathrm{cm}^{2}\right)\end{array}$ & $1.9 \times 10^{2}$ & $1.3 \times 10^{2}$ & $1.2 \times 10^{2}$ & $1.5 \times 10^{2}$ \\
\hline $\begin{array}{c}\text { Series Resistance } \\
(\Omega)\end{array}$ & $1.5 \times 10^{7}$ & $2.1 \times 10^{7}$ & $4.0 \times 10^{7}$ & $2.5 \times 10^{7}$ \\
\hline Ideality factor (n) & 1.6 & 2.0 & 2.0 & 1.9 \\
\hline
\end{tabular}


Investigation of the effect of the contact metal on the organic on inorganic semiconductor heterojunction properties was carried out. The aluminium (Al) anode contact was replaced by lower work function magnesium - silver (Mg-Ag) alloy. The $\mathrm{J}-\mathrm{V}$ characteristic of the MEH-PPV POSS on $\mathrm{n}-\mathrm{Si}$ heterojunctions with magnesium-silver (Mg-Ag) anode was measured and presented in Figures 5.10 and 5.11 with most of the key parameters summarised in Table 5.2. Basically, the heterojunctions with $\mathrm{Mg}-\mathrm{Ag}$ or $\mathrm{Al}$ contact display a similar rectifying characteristic. In Table 5.2, the average rectification ratio $R_{R}$ evaluated at applied voltage of $1 \mathrm{~V}$ and $2 \mathrm{~V}$ are 45 and $1.5^{*} 10^{2}$ respectively. Both values are smaller than when Al contact was used. The average magnitude for the saturation current density $J_{0}$ and reverse leakage current density were determined to be $55 \mathrm{nA} / \mathrm{cm}^{2}$ and $1.5 \times 10^{2} \mu \mathrm{A} / \mathrm{cm}^{2}$. The average series resistance $R_{S}$ was calculated to be around $25 \mathrm{M} \Omega$. The heterojunctions have an average $\mathrm{n}$ factor of 1.9 in region 2 of the semilog $\mathrm{J}-\mathrm{V}$ plot, indicating that recombination current is dominant. Comparing the two tables, it is evident that MEH-PPV POSS on n-Si heterojunction devices seem to perform slightly better as a rectifying junction when $\mathrm{Al}$ anode was used. In Figure 5.12, the energy band diagrams for the MEH-PPV POSS on n-Si heterojunction devices with different anode before and after the Fermi level alignment have been constructed. As shown in the figure, there exists a potential barrier at the interface between the n-Si and MEH-PPV POSS 
polymer. The electrons in forward bias will have to overcome this barrier in order to flow to the opposite terminal. On the other hand, holes injected from the anode will encounter a smaller energy barrier as shown in Figure 5.12 and therefore become the dominate transport carriers for these heterojunction devices. As the $\mathrm{Al}$ anode contact has a reported work function of around $4.28 \mathrm{eV}$ and on the other hand, $\mathrm{Mg}-\mathrm{Ag}$ has a work function of around $3.7 \mathrm{eV}$ [5.8]. The use of the low work function metal contributes a high energy offset between the Fermi energy at the anode and the HOMO level in the MEH-PPV POSS (which is $5.3 \mathrm{eV}$ [5.9]) to result in an increased series resistance as evidenced from Table 5.2. This effectively reduces the number of injected holes at the anode and thus the performance of the devices was affected. A more suitable metal for the anode contact could be Gold $(\mathrm{Au})$ since it has a work function of $5.1 \mathrm{eV}$, which will match closely with the HOMO of the organic polymer MEH-PPV POSS. 


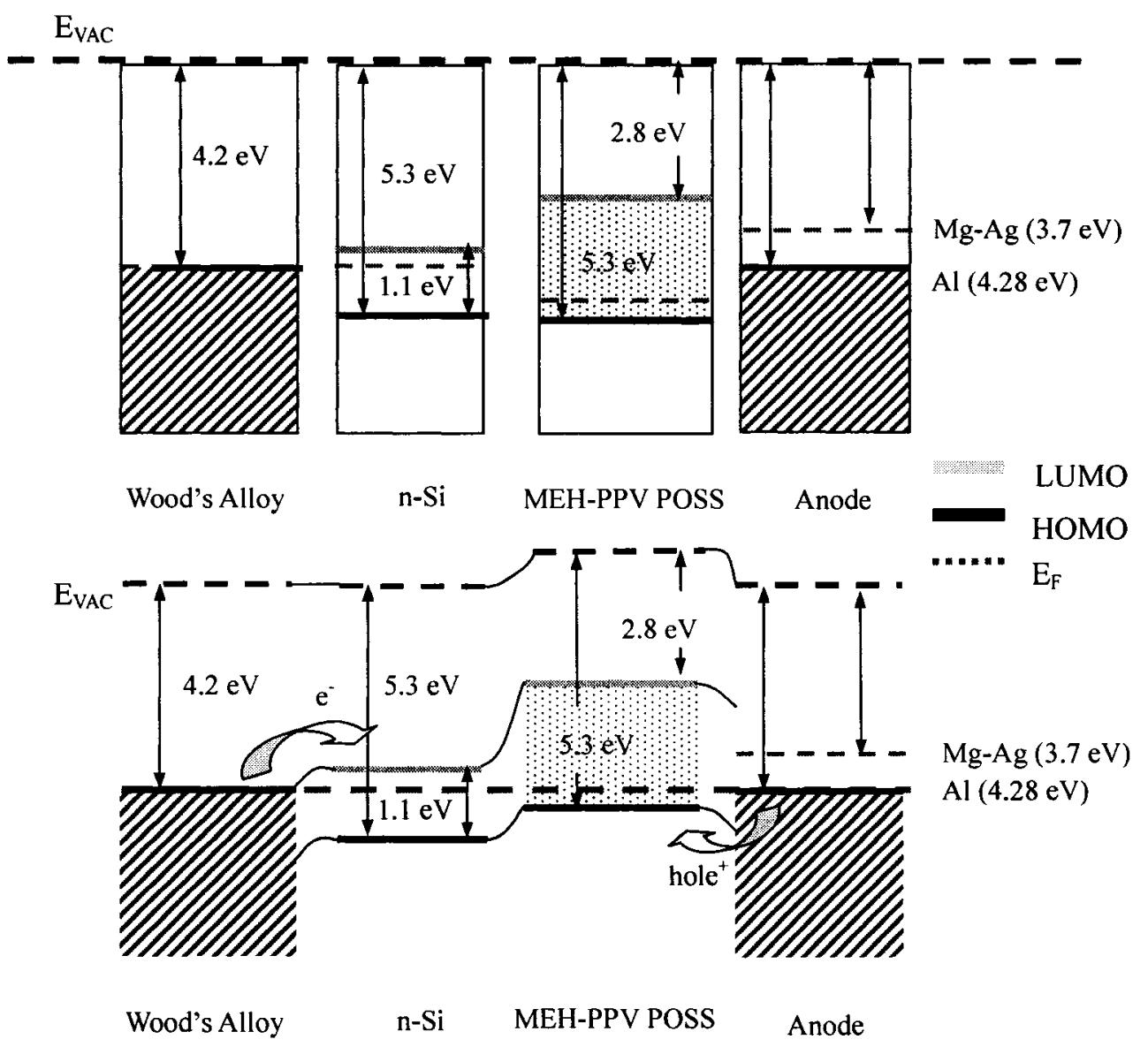

Figure 5.12: (Top) Energy-band diagram for MEH-PPV POSS on n-Si heterojunction devices before Fermi level alignment. (Bottom) Energy-band diagram for MEH-PPV POSS on n-Si heterojunction devices after Fermi level alignment.

\subsubsection{Optical Sensitivity}

Another interesting property of the heterojunction devices that has been investigated was the sensitivity of the devices to optical illumination. Optical sensitivity measurements on Sample SC_06 was carried out and the respective I-V characteristic in forward and reverse bias shown in Figure 5.13 and 5.14. 
Forward In (I) vs Voltage Characteristics for MEH-PPV POSS on n-Si Heterojunctions (with Al contact)

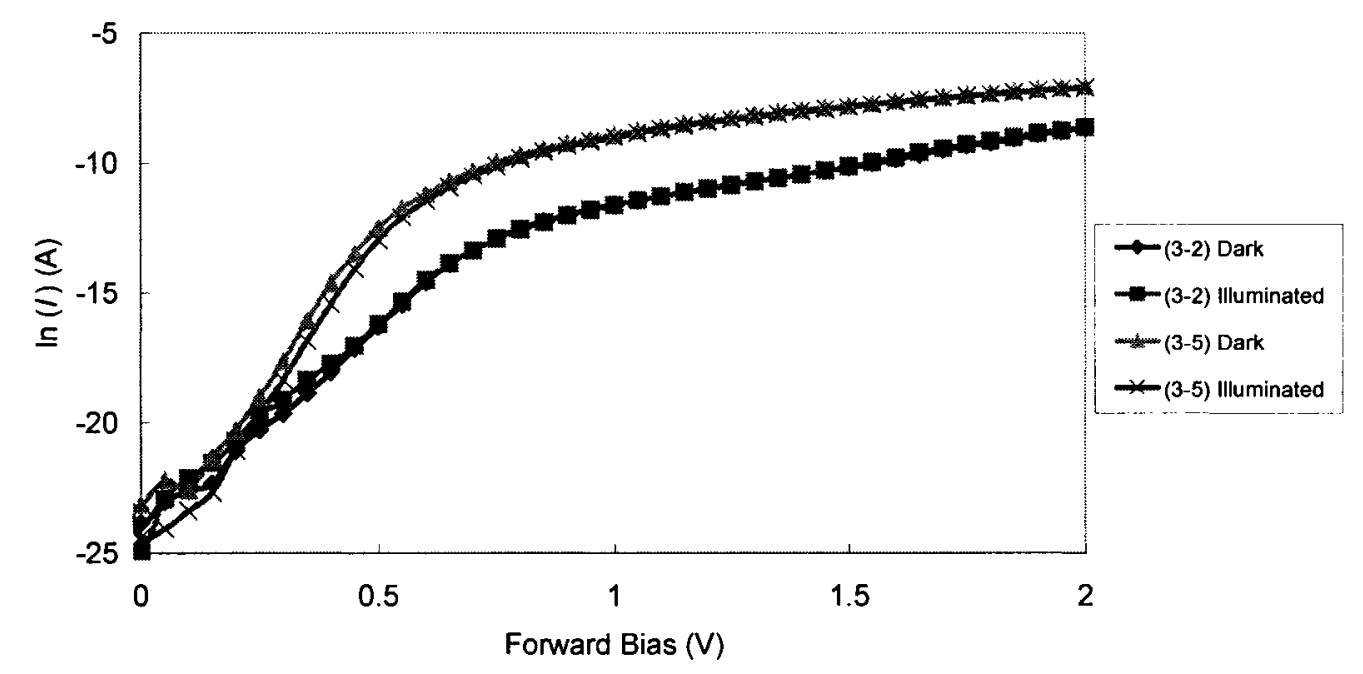

Figure 5.13: Forward $\ln (I)-\mathrm{V}$ characteristics for MEH-PPV POSS on $\mathrm{n}-\mathrm{Si}$ heterojunctions with $\mathrm{Al}$ contact in dark and under illumination.

Reverse In (I) vs Voltage Characteristics for MEH-PPV POSS on n-Si Heterojunctions (with Al contact)

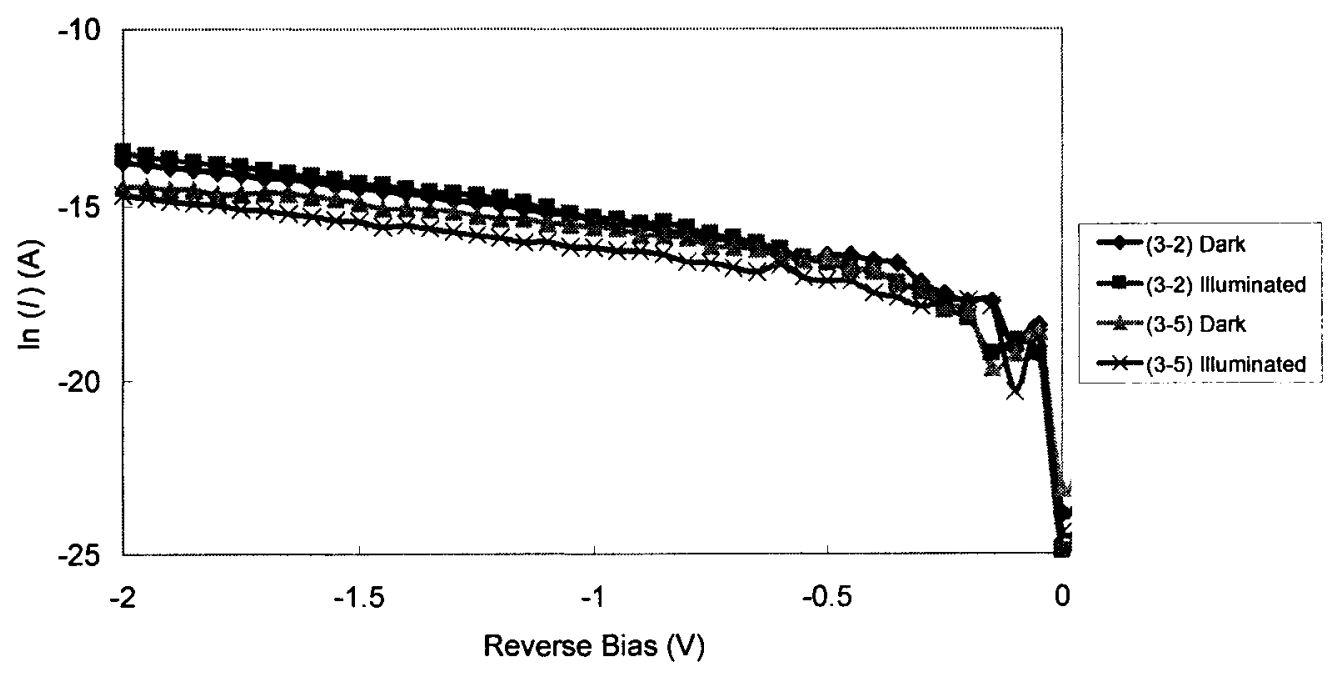

Figure 5.14: Reverse $\ln (I)-\mathrm{V}$ characteristics for MEH-PPV POSS on $\mathrm{n}$-Si heterojunctions with $\mathrm{Al}$ contact in dark and under illumination. 
Table 5.3: Comparison of J-V characteristics for MEH-PPV POSS on n-Si heterojunctions with $\mathrm{Al}$ contact in dark and under illumination

\begin{tabular}{|c|c|c|c|c|}
\hline Organic polymer & \multicolumn{4}{|c|}{ MEH-PPV POSS } \\
\hline $\begin{array}{c}\text { Inorganic } \\
\text { semiconductor }\end{array}$ & \multicolumn{4}{|c|}{ n-type silicon } \\
\hline Anode Contact & \multicolumn{4}{|c|}{ Aluminium } \\
\hline Device Area & \multicolumn{4}{|c|}{$7.9 * 10^{-3} \mathrm{~cm}^{2}$} \\
\hline Sample \# & \multicolumn{4}{|c|}{ SC_06 } \\
\hline Device \# & \multicolumn{2}{|c|}{$3-2$} & \multicolumn{2}{|c|}{$3-5$} \\
\hline Condition & Dark & Illuminated & Dark & Illuminated \\
\hline $\begin{array}{c}\text { Rectification Ratio } \\
\text { (|1V|) }\end{array}$ & $4.5 \times 10$ & $4.1 \times 10$ & $7.9 \times 10^{2}$ & $1.3 \times 10^{2}$ \\
\hline $\begin{array}{c}\text { Rectification Ratio } \\
(|\mathbf{2 V}|)\end{array}$ & $1.6 \times 10^{2}$ & $1.3 \times 10^{2}$ & $1.6 \times 10^{3}$ & $2.0 \times 10^{3}$ \\
\hline $\begin{array}{l}\text { Saturation Current } \\
\text { Density } J_{o}\left(\mathrm{nA} / \mathrm{cm}^{2}\right)\end{array}$ & 48 & 59 & 65 & 53 \\
\hline $\begin{array}{c}\text { Leakage Current } \\
\text { Density }\left(\mu \mathrm{A} / \mathrm{cm}^{2}\right)\end{array}$ & 26 & 29 & 22 & 12 \\
\hline $\begin{array}{c}\text { Series Resistance } \\
(\Omega)\end{array}$ & $3.1 \times 10^{6}$ & $3.0 \times 10^{6}$ & $1.0 \times 10^{6}$ & $1.1 \times 10^{6}$ \\
\hline Ideality factor $(n)$ & 2.2 & 2.3 & 1.4 & 1.4 \\
\hline
\end{tabular}

The illuminated $\mathrm{J}-\mathrm{V}$ characteristics were taken at room temperature under Oriel high power tungsten lamp with an optical power density of $100 \mathrm{~mW} / \mathrm{cm}^{2}$. As shown in Figure 5.13, there is no significant change in the magnitude of the current in dark and under illumination. In Table 5.3, which provides a comparison for devices (3-2) and (3-5) of sample SC_06 under dark and illuminated conditions, the respective parameters for each device are approximately the same. The small differences are within the experimental error range. It seems that the devices are insensitive to optical illumination. However, according to many organic photovoltaic publications, 
photocurrent should be generated at the organic and inorganic heterojunction when the devices are illuminated. Due to the high series resistance speculated to exist between the organic polymer and the anode; the photocurrent might be too small to be measured by the equipment utilized. Futhermore, the opaque contact on the polymer might have limited the amount of light received. This could be why the performance of the MEH-PPV POSS on n-Si heterojunctions appeared to be unaffected by light.

\subsubsection{Regioregular poly(3-hexylthiophene)}

Polythiophenes constitute a particularly important class of conjugated polymers, which has been extensively studied for its optical and electronic properties. Poly(3-alkythiophene)s (P3AT)s including poly(3-hexylthiophene) (P3HT) hold a greater promise in thin film transistor processing, because the alkyl side chain offer improved solubility in many common organic solvent. Furthermore, P3HT has a low bandgap $(1.9 \mathrm{eV})$ compared to other conjugated polymers and a high degree of intermolecular order leading to high charge carrier mobilities $\left(\mu_{\text {hole }} \sim 0.1 \mathrm{~cm}^{2} / \mathrm{Vs}\right.$ measured in field effect transistors) [5.10]. The 3-hexyl groups of the repeated thiophene rings in a P3HT chain can be incorporated into two different regioregularities: head-to-tail $(\mathrm{HT})$ and head-to-head $(\mathrm{HH})$, resulting in four triad regioisomers in the polymer chain (HT-HT, TT-HT, HT-HH and TT-HH triad). These are shown in Figure $5.15(\mathrm{a} \sim \mathrm{d})$. 


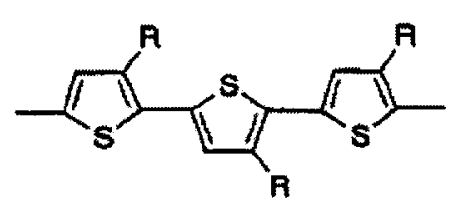

(a) HT-HT

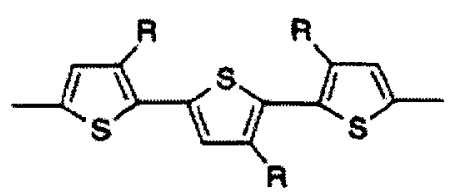

(c) HT-HH

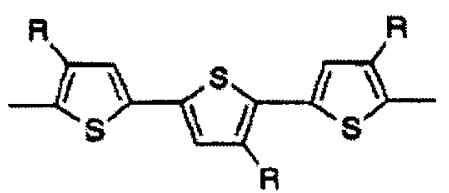

(b) TT-HT

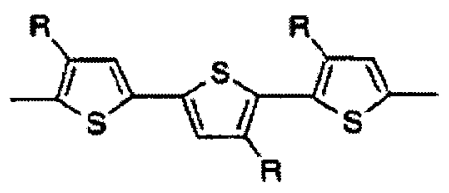

(d) TT-BH

Figure 5.15: Regioisomers in P3HT polymer chain: (a) HT-HT coupling; (b) TT-HT coupling; (c) HT-HH coupling; (d) TT-HH coupling. [5.11]

As $\mathrm{HH}$ linkages have pronounced steric hindrance between opposing hexyl groups, they offer "defects" in molecular ordering of conjugated P3HT chains. Therefore, if P3HT consists of both HH and HT 3-alkylthiophene functional groups, it is called regio-random $\mathrm{P} 3 \mathrm{HT}$, which can only form amorphous films with low field effect mobility $\left(10^{-5} \sim 10^{-4} \mathrm{~cm}^{2} / \mathrm{V}-\mathrm{s}\right)$ due to charge transport limitation in disordered matrix [5.11]. Figure 5.16(a) shows a regio-random P3HT with HT and HH coupling.
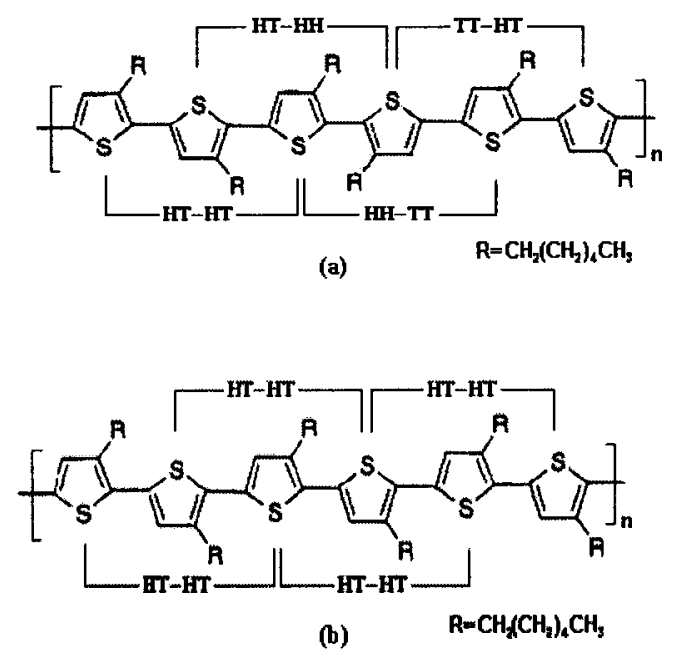

Figure 5.16: (a) Regio-random P3HT with both $\mathrm{HT}$ and $\mathrm{HH}$ coupling; (b) regio-regular P3HT with only HT coupling. [5.11] 
On the other hand, P3HT polymers mediated by Rieke zinc [5.12] can be controlled to maintain high HT regioregularity (>98.5\% HT linkages) in polymer chain. Furthermore, these regioregular-P3HT (RR-P3HT) can self-orient into a well-ordered lamellar structure, with molecular chains parallel to the substrate, and thiophene rings perpendicular to the substrate, resulting in high field-effect mobility $\left(10^{-2} \mathrm{~cm}^{2} / \mathrm{V}-\mathrm{s}\right)$ [5.13]. The high mobility is due to the high degree of crystallinity and strong interchain interactions. Regioregular P3HT with only HT coupling is shown in Figure 5.16(b). RR-P3HT solution in this work was prepared by dissolving $56 \mathrm{mg}$ of the organic polymer in $8 \mathrm{~g}$ of chloroform to obtain a concentration of $0.7 \mathrm{wt} \%$.

\subsubsection{Current-Voltage Characteristics}

RR-P3HT on n-Si heterojunction devices with Al contact were fabricated using the procedure described in Chapter 4 but with a small variation in the structure of the device. Figure 5.17 shows the schematic diagram of the actual heterojunction devices fabricated and the energy-band diagrams have been constructed in Figure 5.18. The corresponding I-V characteristics in forward and reverse bias are shown in Figure $5.19 \& 5.20$ while some of the key parameters summarised in Table 5.4. 


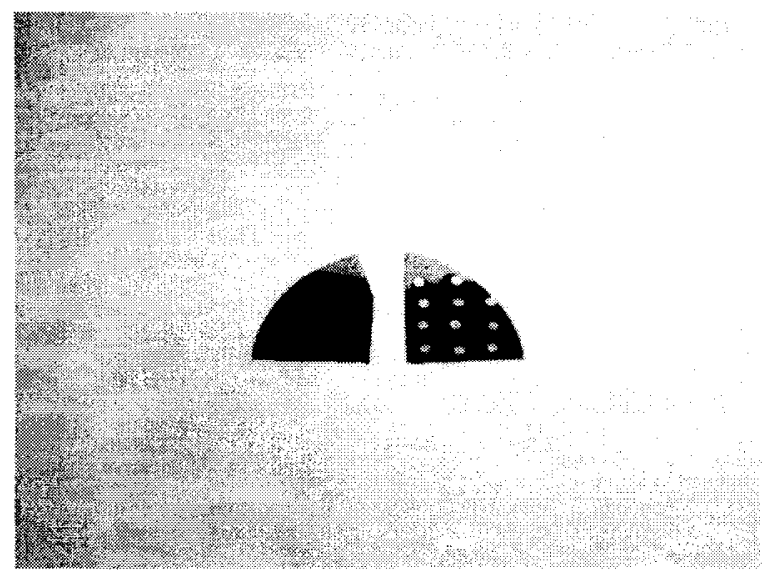

Figure 5.17: Photograph of RR-P3HT on n-Si heterojunction devices.

$\mathrm{E}_{\mathrm{VAC}}$
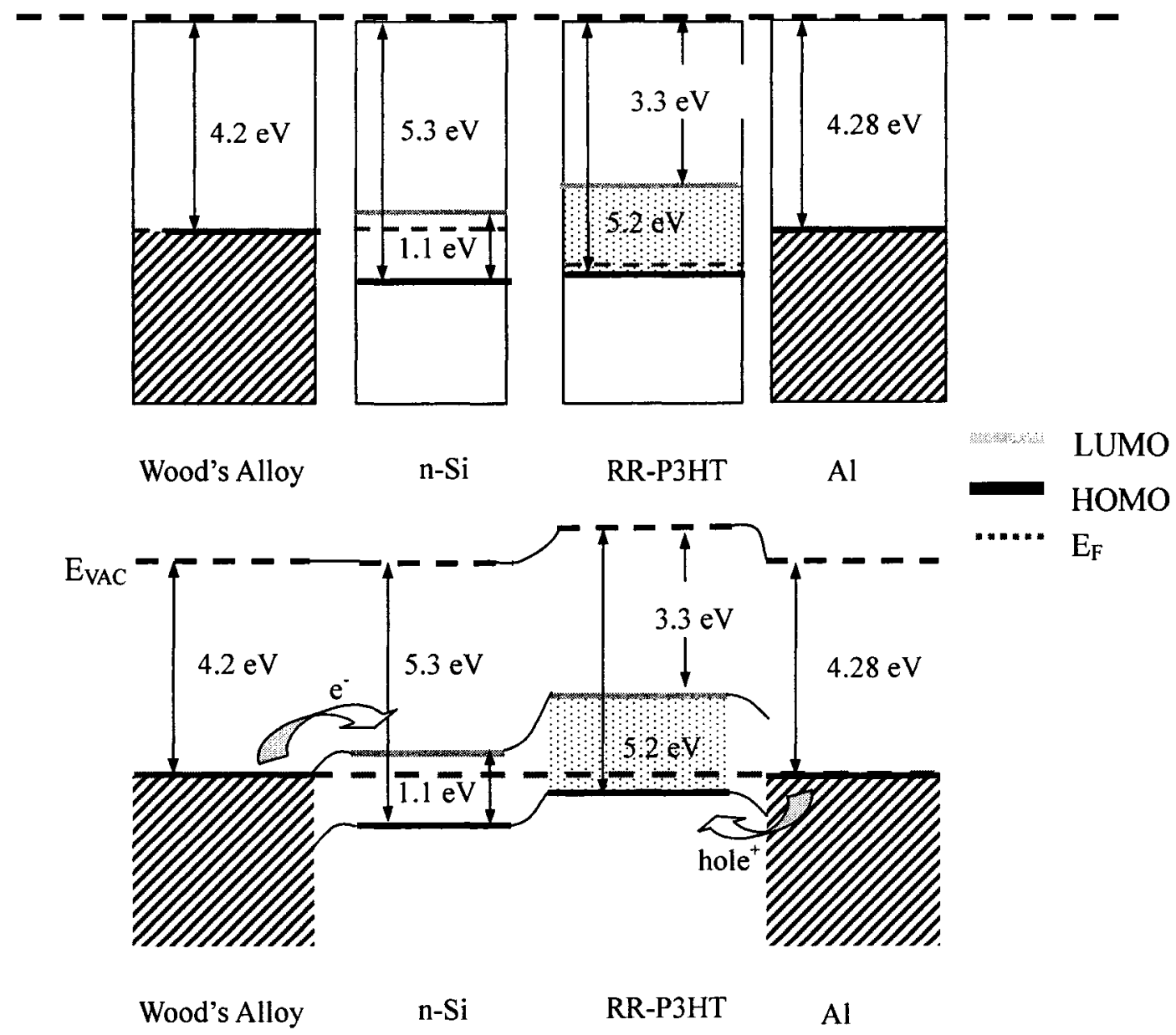

Figure 5.18: (Top) Energy-band diagram for RR-P3HT on n-Si heterojunction devices before Fermi level alignment. (Bottom) Energy-band diagram for RR-P3HT on n-Si heterojunction devices after Fermi level alignment. 
Forward in (I) vs Voltage Characteristics for P3HT on n-Si Heterojunctions (with Al contact)

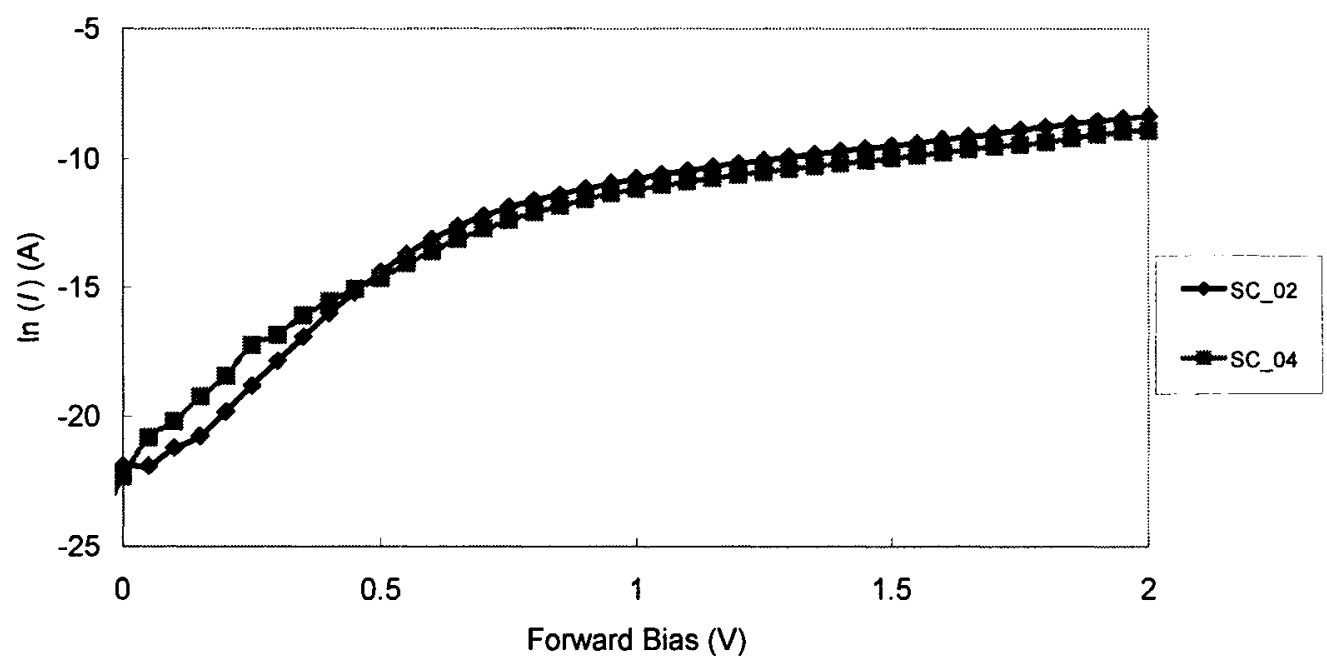

Figure 5.19: $\ln (I)-\mathrm{V}$ characteristics for RR-P3HT on n-Si heterojunctions with Al contact in forward bias

Reverse In(I) vs Voltage Characteristics for P3HT on n-Si Heterojunctions (with Al contact)

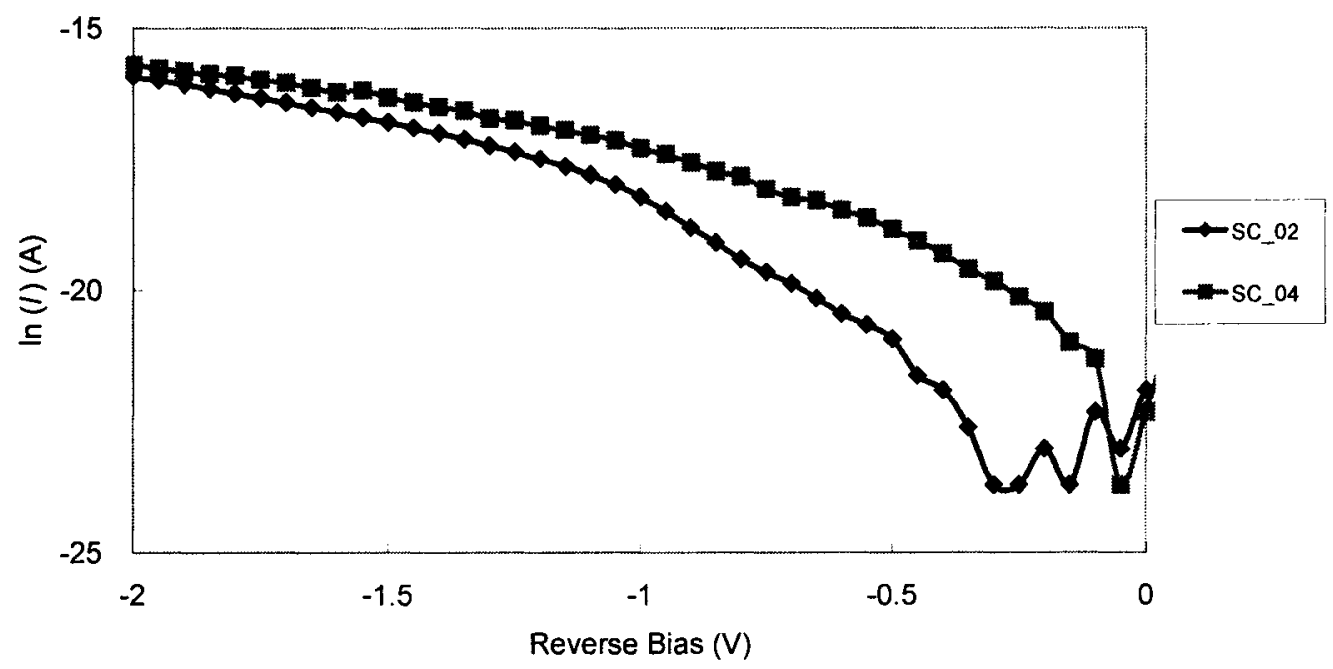

Figure 5.20: $\ln (I)-\mathrm{V}$ characteristics for RR-P3HT on n-Si heterojunctions with $\mathrm{Al}$ contact in reverse bias 
Table 5.4: Summary of J-V characteristic for RR-P3HT on n-Si

Heterojunction devices with Al contact

\begin{tabular}{|c|c|c|c|}
\hline Organic polymer & \multicolumn{3}{|c|}{ P3HT } \\
\hline $\begin{array}{c}\text { Inorganic } \\
\text { semiconductor }\end{array}$ & \multicolumn{3}{|c|}{ n-type silicon } \\
\hline Cathode Contact & \multicolumn{3}{|c|}{ Aluminium } \\
\hline Device Area & \multicolumn{3}{|c|}{$7.9 \times 10^{-3} \mathrm{~cm}^{2}$} \\
\hline Device \# & $S C_{-} 02$ & $S C \_04$ & Average \\
\hline $\begin{array}{c}\text { Rectification Ratio } \\
(|\mathbf{1 V}|)\end{array}$ & $1.8 \times 10^{3}$ & $4.6 \times 10^{2}$ & $1.1 \times 10^{3}$ \\
\hline $\begin{array}{c}\text { Rectification Ratio } \\
(|2 V|)\end{array}$ & $1.9 \times 10^{3}$ & $8.9 \times 10^{2}$ & $1.4 \times 10^{3}$ \\
\hline $\begin{array}{l}\text { Saturation Current } \\
\text { Density } J_{0}\left(\mathrm{nA} / \mathrm{cm}^{2}\right)\end{array}$ & 7.9 & 36 & 22 \\
\hline $\begin{array}{c}\text { Leakage Current } \\
\text { Density }\left(\mu \mathrm{A} / \mathrm{cm}^{2}\right)\end{array}$ & 1.6 & 3.9 & 2.8 \\
\hline $\begin{array}{c}\text { Series Resistance } \\
(\Omega)\end{array}$ & $2.7 \times 10^{6}$ & $4.9 \times 10^{6}$ & $3.8 \times 10^{6}$ \\
\hline Ideality factor (n) & 2.0 & 1.9 & 2.0 \\
\hline
\end{tabular}

From Figure 5.19, the RR-P3HT on n-Si heterojunction devices showed a similar rectifying behaviour indicating the presence of a potential barrier. In Table 5.4, the rectification ratio $R_{R}$ was evaluated at applied voltage of $1 \mathrm{~V}$ and $2 \mathrm{~V}$ respectively. The average value of $R_{R}$ for RR-P3HT on $n-S i$ heterojunctions with aluminium anode, are $1.1 \times 10^{3}$ (at $1 \mathrm{~V}$ ) and $1.4 \times 10^{3}$ (at $2 \mathrm{~V}$ ) respectively. These values are almost 1 to 2 orders of magnitude larger than the MEH-PPV POSS on n-Si heterojunctions. This could be due to slightly lower energy barrier height encountered by the electrons in forward bias operation of the P3HT on n-Si heterojunction devices as shown in Figure 5.18. The average values for the saturation current density $\mathrm{J}_{0}$ and reverse leakage 
current density were determined to be $22 \mathrm{nA} / \mathrm{cm}^{2}$ and $2.8 \mu \mathrm{A} / \mathrm{cm}^{2}$ respectively. Moreover, the average series resistance $R_{S}$ was calculated to be around $3.8 \mathrm{M} \Omega$. The $\mathrm{n}$ factors listed in Table 5.4 are calculated using the current measured from region 2 of the heterojunction. The fabricated heterojunctions have $\mathrm{n}$ greater than 2 in the small forward bias region, indicating that recombination current is dominant. For region $2, \mathrm{n}$ factor is around 2, indicating a recombination current component is still dominant. At high voltages, $\mathrm{n}$ factor becomes extremely large due to the series resistance effect.

Similar to MEH-PPV polymer, RR-P3HT has a reported HOMO of $5.2 \mathrm{eV}$ while work function of the $\mathrm{Al}$ anode is $4.28 \mathrm{eV}$. Therefore, there exists an energy-level offset at the contact and polymer interface which will affect the hole-injection efficiency and thus performance of the devices. Furthermore, known disadvantage of the P3HT polymers such as sensitivity to oxygen and moisture [5.14] could lead to the degradation phenomenon observed from some of the devices as the measurement were carried out in air at room temperature over a period of time. Further investigation also indicated that the RR-P3HT on $\mathrm{n}-\mathrm{Si}$ heterojunction devices appeared to be insensitive to illumination.

\subsubsection{PEDOT}

In recent years, poly(3,4-ethylenedioxythiophene) (PEDOT) has been of considerable interest because of the possibility of producing a material with linear 
chains [5.15]. PEDOT is a conducting polymer based on a heterocyclic thiophene ring bridged by a diether. This means it has the same conjugated backbone as polythiophene. PEDOT has excellent transparency in the visible region, good electrical conductivity, and environmental stability. Unfortunately PEDOT, like most conducting polymers, is infusible and insoluble and therefore difficult to process in a thin-film form or in other shapes. However, if PEDOT is electrochemically polymerized from aqueous solutions doped with poly(styrene sulfonate) (PSS), [5.16] it will become more easily processibile. A conductive layer of PEDOT:PSS is obtained by spreading a layer of the dispersion on the surface usually by spin-coating and drying out the water by heat. It can be spin-coated onto a huge variety of conducting and non-conductive substrates including glass, silicon, chromium, gold, etc. Chemical structure of PEDOT:PSS is shown in Figure 5.21.

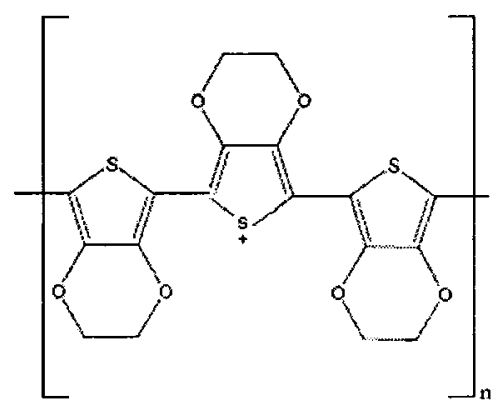<smiles>CCC(C)c1ccc(O)cc1</smiles><smiles>CCC(C)c1ccc(S(=O)(=O)O)cc1</smiles>

Figure 5.21: Chemical structure of conducting polymer PEDOT:PSS from Aldrich.

This polymer was first used for the antistatic equipment of photographic materials [5.17]. Nowadays PEDOT:PSS serves as a buffer layer in OLED to improve 
hole injection. These buffer layers have a high morphological and redox stability and very good film forming properties [5.18], by which the rough surface of optical transparent electrodes is smoothed out. The PEDOT in this work was purchased from Sigma Aldrich (product \# 483095).

\subsubsection{Current-Voltage Characteristics}

PEDOT on $\mathrm{n}-\mathrm{Si}$ heterojunction devices were fabricated using the procedure described in Chapter 4. Each device has a circular active area of roughly $1 \times 10^{-2} \mathrm{~cm}^{2}$. The first set of heterojunctions was fabricated with ordinary PEDOT while the PEDOT of the second set of devices was doped (30\%) with a p-type dopant boron trifluoride $\left(\mathrm{BF}_{3}\right)$. Figure 5.22 shows a picture of the actual heterojunction devices fabricated and the associated energy-band diagrams have been constructed in Figure 5.23. I-V characteristics of the corresponding devices and a summary of their key parameters will be presented in the following figures and tables below.

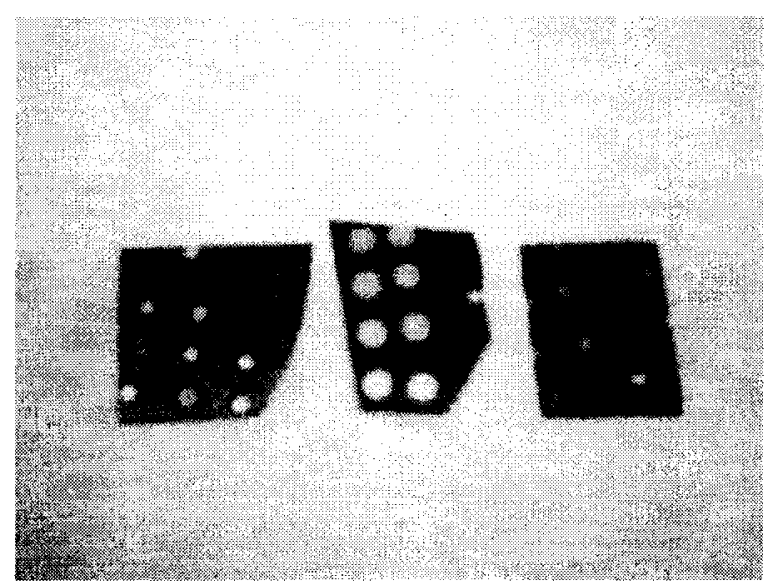

Figure 5.22: A photograph showing heterojunction devices of the PEDOT on n-Si. 


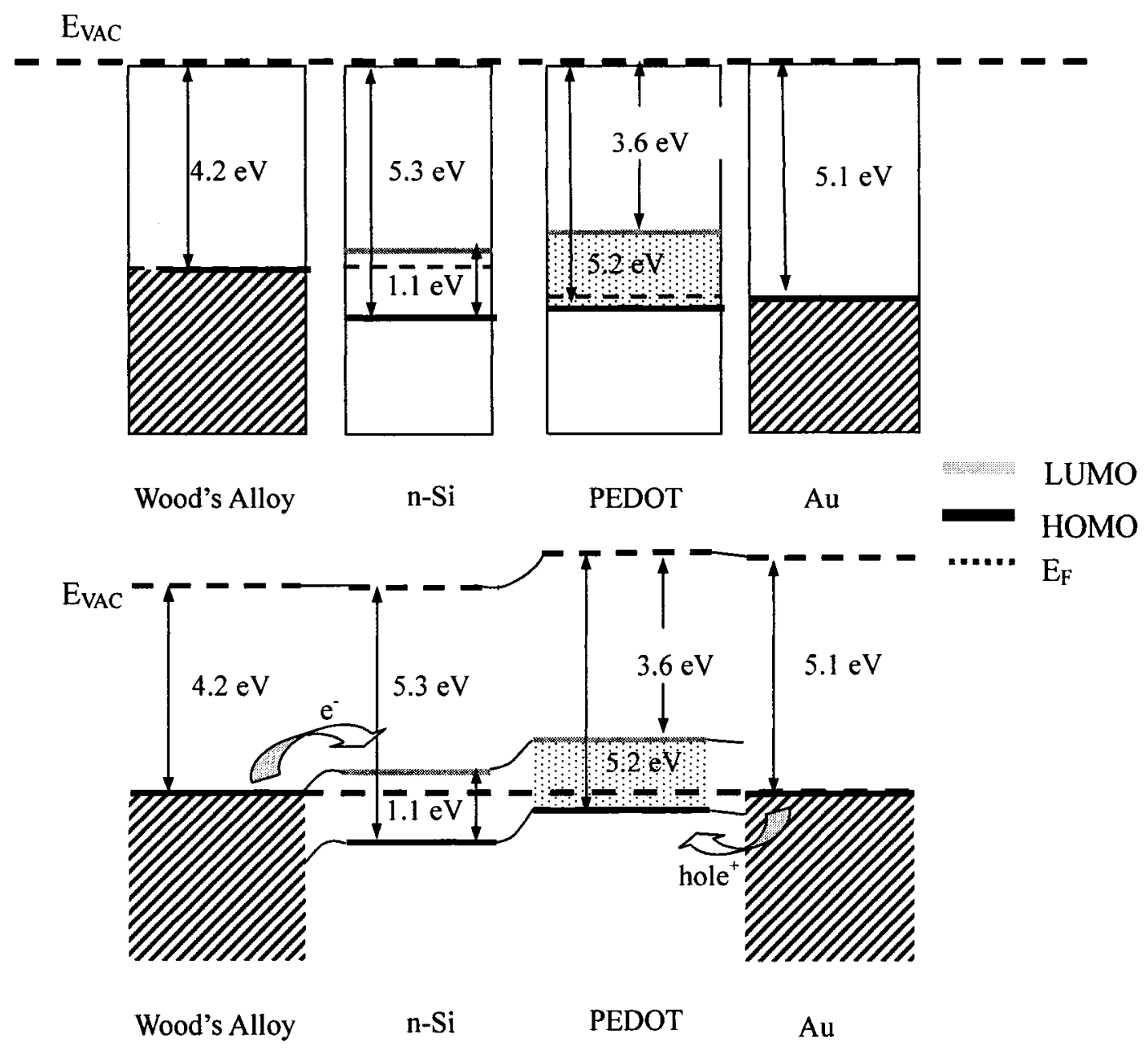

Figure 5.23: (Top) Energy-band diagram for PEDOT on n-Si heterojunction devices before Fermi level alignment. (Bottom) Energy-band diagram for PEDOT on n-Si heterojunction devices after Fermi level alignment. 


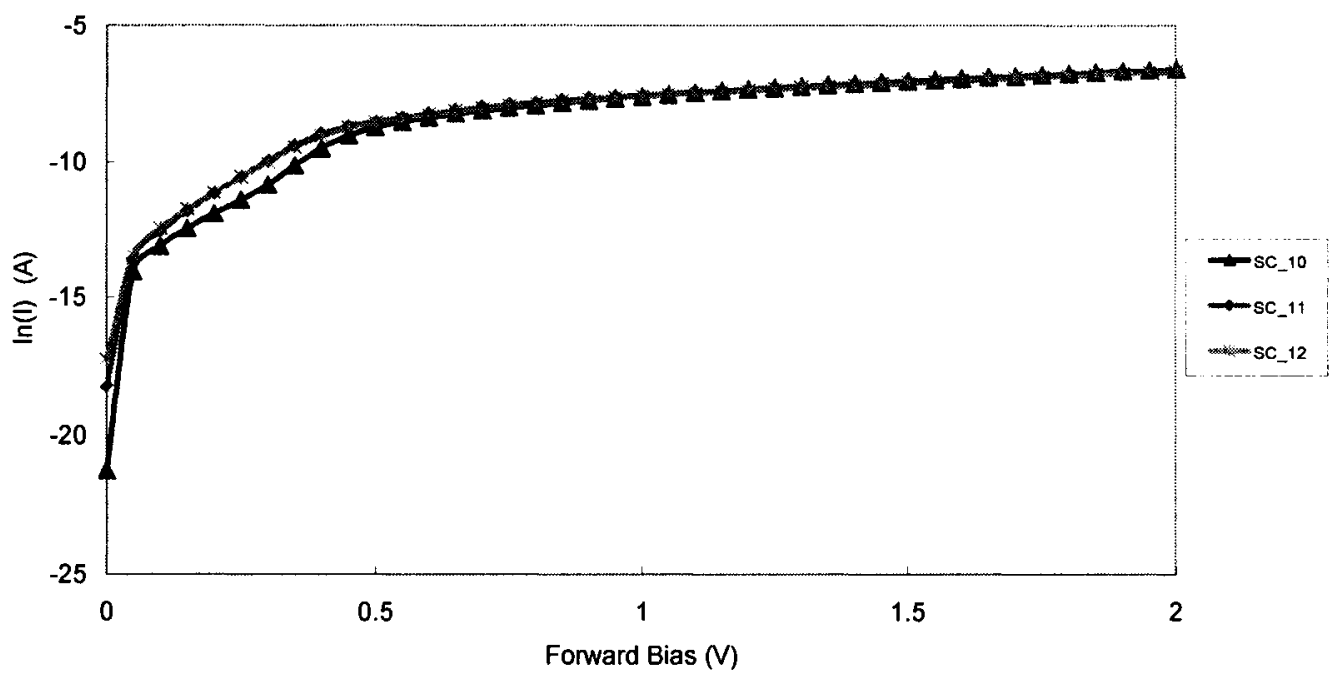

Figure 5.24: $\ln (I)-\mathrm{V}$ characteristics for PEDOT on $\mathrm{n}-\mathrm{Si}$ heterojunctions with $\mathrm{Au}$ contact in forward bias

Table 5.5: Summary of the J-V characteristic for PEDOT on n-Si heterojunctions with Au contact

\begin{tabular}{|c|c|c|c|c|}
\hline Organic polymer & \multicolumn{4}{|c|}{ PEDOT } \\
\hline $\begin{array}{c}\text { Inorganic } \\
\text { semiconductor }\end{array}$ & \multicolumn{4}{|c|}{ n-type silicon } \\
\hline Anode Contact & \multicolumn{4}{|c|}{ Gold } \\
\hline Device Area & \multicolumn{4}{|c|}{$1 \times 10^{-2} \mathrm{~cm}^{2}$} \\
\hline Sample \# & $S C_{-} 10$ & $S C \_11$ & $S C_{-} 12$ & Average \\
\hline $\begin{array}{c}\text { Rectification Ratio } \\
(|\mathbf{1 V}|)\end{array}$ & $1.6 \times 10^{4}$ & $1.2 \times 10^{4}$ & $9.3 \times 10^{3}$ & $1.2 \times 10^{4}$ \\
\hline $\begin{array}{c}\text { Rectification Ratio } \\
(|2 \mathrm{2}|)\end{array}$ & $2.7 \times 10^{4}$ & $1.9 \times 10^{4}$ & $1.4 \times 10^{4}$ & $2.0 \times 10^{4}$ \\
\hline $\begin{array}{c}\text { Saturation Current } \\
\text { Density } J_{0}\left(\mu A / \mathbf{c m}^{2}\right)\end{array}$ & 8.3 & 14 & 23 & 15 \\
\hline $\begin{array}{c}\text { Leakage Current } \\
\text { Density }\left(\mu \mathrm{A} / \mathrm{cm}^{2}\right)\end{array}$ & 0.33 & 0.45 & 0.55 & 0.44 \\
\hline $\begin{array}{c}\text { Series Resistance } \\
(\Omega)\end{array}$ & $9.2 \times 10^{5}$ & $9.6 \times 10^{5}$ & $1.0 \times 10^{5}$ & $9.6 \times 10^{5}$ \\
\hline Ideality factor (n) & 3.2 & 2.7 & 2.9 & 2.9 \\
\hline
\end{tabular}


From the $\mathrm{J}-\mathrm{V}$ data collected in Figures 5.24, the PEDOT on n-Si heterojunction devices clearly display a rectifying characteristic. The rectification ratio is larger than that of the MEH-PPV POSS or RR-P3HT heterojunction devices. From Table 5.5, the average rectification ratio at $1 \mathrm{~V}$ and $2 \mathrm{~V}$ for these PEDOT heterojunctions are $1.2 \times 10^{4}$ and $2.0 \times 10^{4}$ respectively, which are a few orders of magnitude larger than the previous 2 sets of samples. The large $R_{R}$ could be due to the small energy barrier height encountered by the electrons of the PEDOT on $\mathrm{n}-\mathrm{Si}$ heterojunction devices during forward bias operation. Referring to Figure 5.23, both the electrons and holes only have to overcome a smaller energy barrier when compared with the other two heterojunctions, therefore, both electrons and holes will contribute to the charge transport. The corresponding average leakage current density in reverse bias is $0.44 \mu$ $\mathrm{A} / \mathrm{cm}^{2}$. Average saturation current density, determined from the $y$-intercept of a line drawn according to the slope of the J-V curves of the PEDOT on n-Si heterojunction devices, is found to be $15 \mu \mathrm{A} / \mathrm{cm}^{2}$. The average series resistance calculated is around $0.96 \mathrm{M} \Omega$. One interesting observation from the $\mathrm{J}-\mathrm{V}$ characteristics of the PEDOT on $\mathrm{n}$-Si heterojunction devices is that at very small forward bias $(0 \sim 0.05 \mathrm{~V})$, the devices have an average $n$-factor of 0.4 . This phenomenon did not occur in the previous two sets of devices with different organic polymers. Usually, for non-ideal p-n junctions under small forward bias, they have an ideality factor of 2 or greater, indicating the 
recombination current component is dominant. However, it is totally the opposite in this case. The explanation for this observation is still unclear. As the forward voltage bias was increased, the $\mathrm{n}$ factor increased slowly as well. The average $\mathrm{n}$ factor calculated in region 2 is 2.9 . This means that the recombination current component in the total current is dominant. At high current injection, the $\mathrm{n}$ factor grows extremely large due to the series resistance effect.

Boron atom is a common p-type dopant used in the semiconductor industry. PEDOT on the other hand has been the most widely used hole-injecting material in OLED research and development. In the next section, effect of boron doping on the properties of PEDOT-Si heterojunction will be investigated. Figure 5.25 shows the $\mathrm{J}-\mathrm{V}$ characteristics of the PEDOT:BF $\mathrm{B}_{3}$ on $\mathrm{n}-\mathrm{Si}$ heterojunction devices.

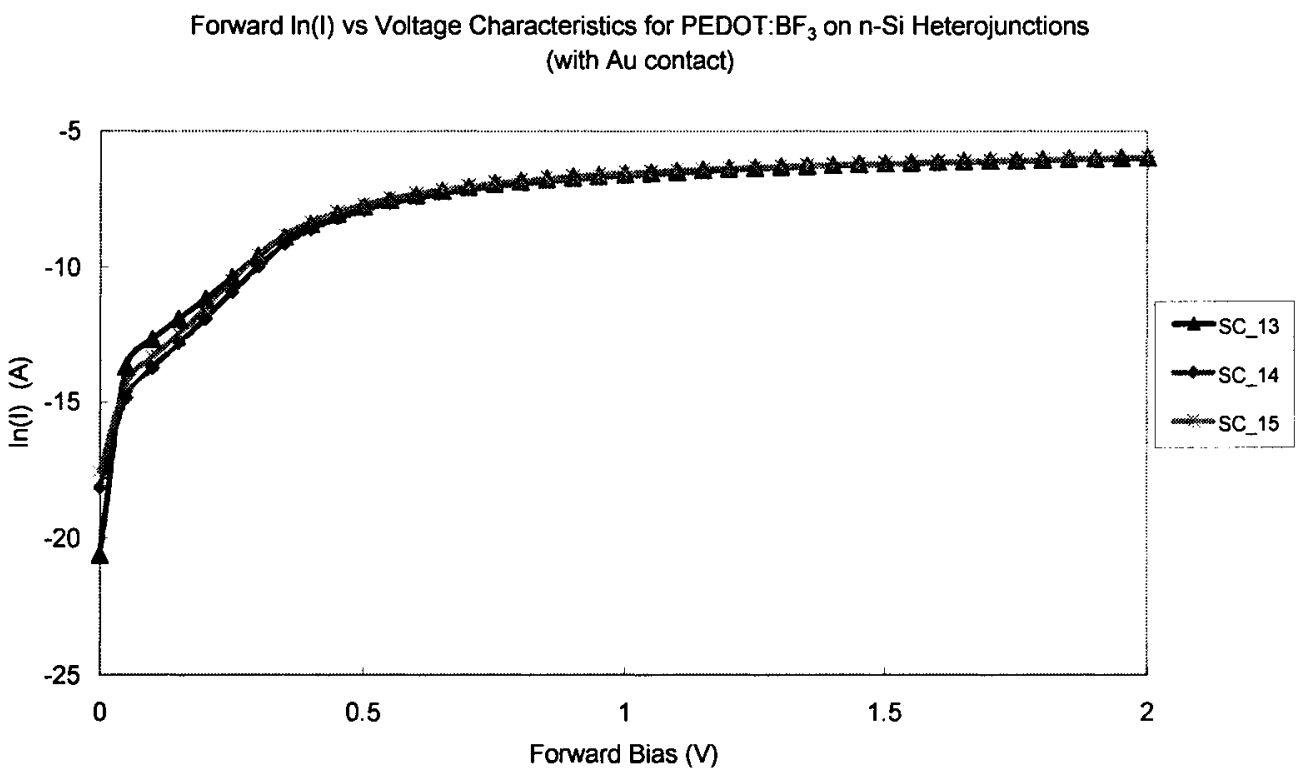

Figure 5.25: $\ln (I)-\mathrm{V}$ characteristics for PEDOT: $\mathrm{BF}_{3}$ on $\mathrm{n}-\mathrm{Si}$ heterojunctions with $\mathrm{Au}$ contact in forward bias 


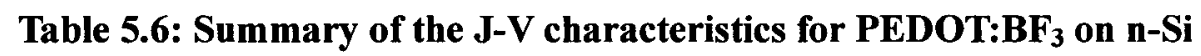

heterojunctions with Au contact

\begin{tabular}{|c|c|c|c|c|}
\hline Organic polymer & \multicolumn{4}{|c|}{ PEDOT:BF 3} \\
\hline $\begin{array}{c}\text { Inorganic } \\
\text { semiconductor }\end{array}$ & \multicolumn{4}{|c|}{ n-type silicon } \\
\hline Anode Contact & \multicolumn{4}{|c|}{ Gold } \\
\hline Device Area & \multicolumn{4}{|c|}{$1 \times 10^{-2} \mathrm{~cm}^{2}$} \\
\hline Sample \# & $S C_{-} 13$ & $S C_{-} 14$ & $S C_{-} 15$ & Average \\
\hline $\begin{array}{c}\text { Rectification Ratio } \\
(|\mathbf{1 V}|)\end{array}$ & $5.3 \times 10^{4}$ & $6.1 \times 10^{4}$ & $3.9 \times 10^{4}$ & $5.1 \times 10^{4}$ \\
\hline $\begin{array}{c}\text { Rectification Ratio } \\
(|2 \mathrm{~V}|)\end{array}$ & $5.9 \times 10^{4}$ & $6.6 \times 10^{4}$ & $4.7 \times 10^{4}$ & $5.7 \times 10^{4}$ \\
\hline $\begin{array}{c}\text { Saturation Current } \\
\text { Density } J_{0}\left(\mu A / \mathrm{cm}^{2}\right)\end{array}$ & 5 & 14 & 10 & 9.7 \\
\hline $\begin{array}{c}\text { Leakage Current } \\
\text { Density }\left(\mu \mathrm{A} / \mathrm{cm}^{2}\right)\end{array}$ & 0.27 & 0.22 & 0.37 & 0.29 \\
\hline $\begin{array}{c}\text { Series Resistance } \\
(\Omega)\end{array}$ & $9.9 \times 10^{5}$ & $1.0 \times 10^{6}$ & $9.9 \times 10^{5}$ & $9.9 \times 10^{5}$ \\
\hline Ideality factor (n) & 2.6 & 2.1 & 2.2 & 2.3 \\
\hline
\end{tabular}

From Figure 5.25 , the $\mathrm{J}-\mathrm{V}$ characteristics obtained for the PEDOT:BF $\mathrm{B}_{3}$ on $\mathrm{n}-\mathrm{Si}$

heterojunction devices were similar to that of just PEDOT on n-Si. They both have three distinctive regions of slopes or $\mathrm{n}$ factor. The PEDOT:BF $\mathrm{B}_{3}$ devices displayed a better rectifying characteristic with $\mathrm{R}_{\mathrm{R}}$ of $5.1 \times 10^{4}(@|1 \mathrm{~V}|)$ and $5.7 \times 10^{4}(@|2 \mathrm{~V}|$ ) shown in Table 5.6 respectively. The rectification ratios are a few times larger than the corresponding values obtained for PEDOT on n-Si heterojunctions. This could be due to the smaller leakage current in reverse bias when PEDOT was doped with $\mathrm{BF}_{3}$, it has an average leakage current density of $0.29 \mu \mathrm{A} / \mathrm{cm}^{2}$. The corresponding saturation current density $I_{0}$ is $9.7 \mu \mathrm{A} / \mathrm{cm}^{2}$ while the average series resistance is $0.99 \mathrm{M} \Omega$. The 
average $\mathrm{n}$ factor calculated for region 2 of the J-V characteristics is around 2.3, which is slightly smaller than 2.9 obtained from the previous set of devices. Once again, at extremely small forward bias $(0 \sim 0.05 \mathrm{~V})$, the $\mathrm{J}-\mathrm{V}$ characteristic displayed a very steep slope thus an $\mathrm{n}$-factor of 0.48 , which is consistent with the result obtained for the set without $\mathrm{BF}_{3}$ doping. One interesting observation is that the total output current density for the PEDOT:BF 3 on n-Si heterojunction devices is almost $2 \sim 3$ times larger than that of the PEDOT on n-Si heterojunction devices as shown in Figure 5.26.

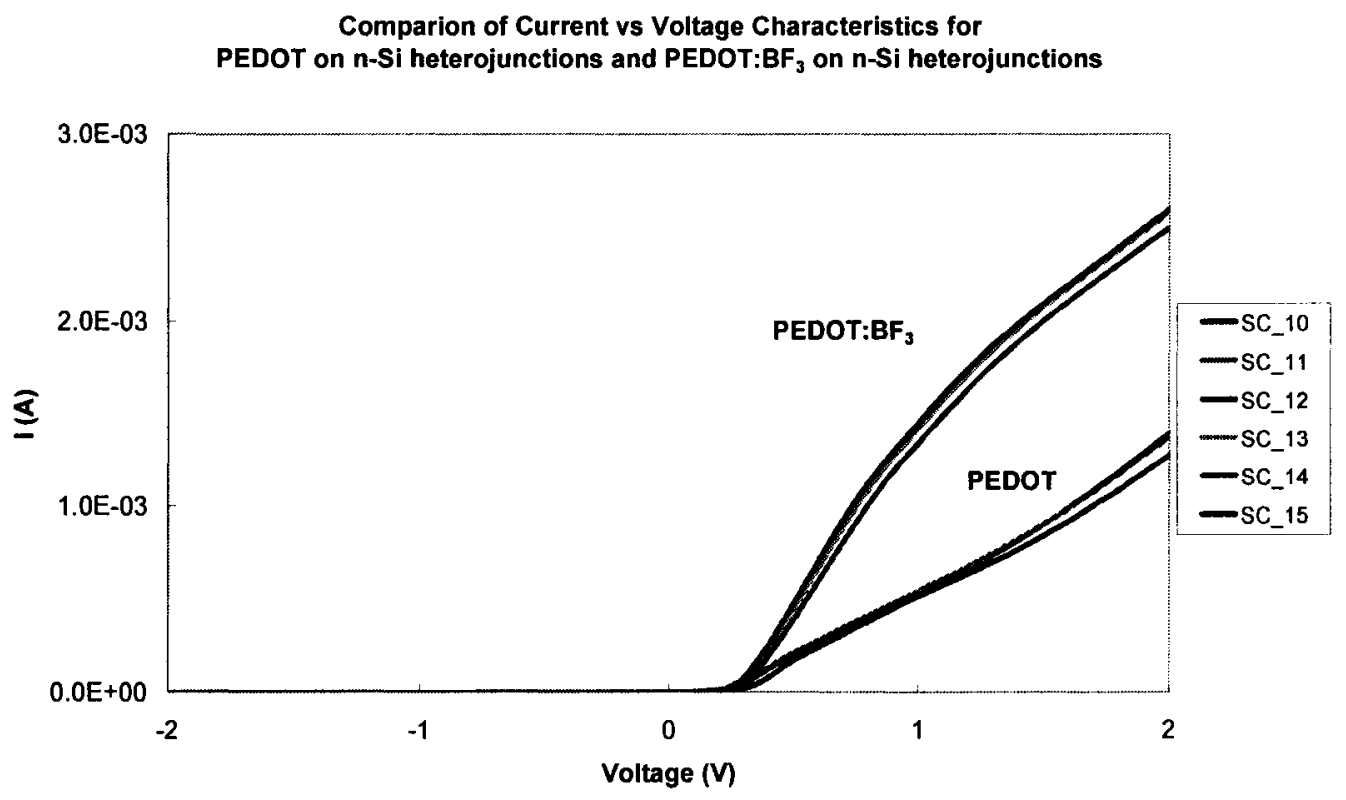

Figure 5.26: Comparison of the I-V characteristics for PEDOT on $\mathrm{n}-\mathrm{Si}$ heterojunctions and PEDOT:BF 3 on n-Si heterojunctions. 


\subsubsection{Capacitance-Voltage Characteristics}

For organic polymer on inorganic semiconductor heterojunctions, measurements of the differential capacitance - voltage can provide knowledge about the carrier concentration and built-in voltage. As explained in section 5.2.2, any variation of the charge within a p-n diode with an applied voltage variation yields a capacitance which must be added to the circuit model of a p-n diode. The capacitance associated with the charge variation in the depletion layer is called the junction capacitance, while the capacitance associated with the excess carriers in the quasi-neutral region is called diffusion capacitance. The junction capacitance dominates for the reversed-biased diodes, while the diffusion capacitance dominates in strongly forward-biased diodes. Figure 5.27 illustrates the variation of the differential junction capacitance with the bias voltage at a frequency of $100 \mathrm{kHz}$ for two sets of 3 PEDOT on $\mathrm{n}-\mathrm{Si}$ heterojunction (SC_10 12) and 3 PEDOT: $\mathrm{BF}_{3}$ on $\mathrm{n}-\mathrm{Si}$ heterojunction $\left(\mathrm{SC}_{-} 13 \sim 15\right)$ devices respectively. 


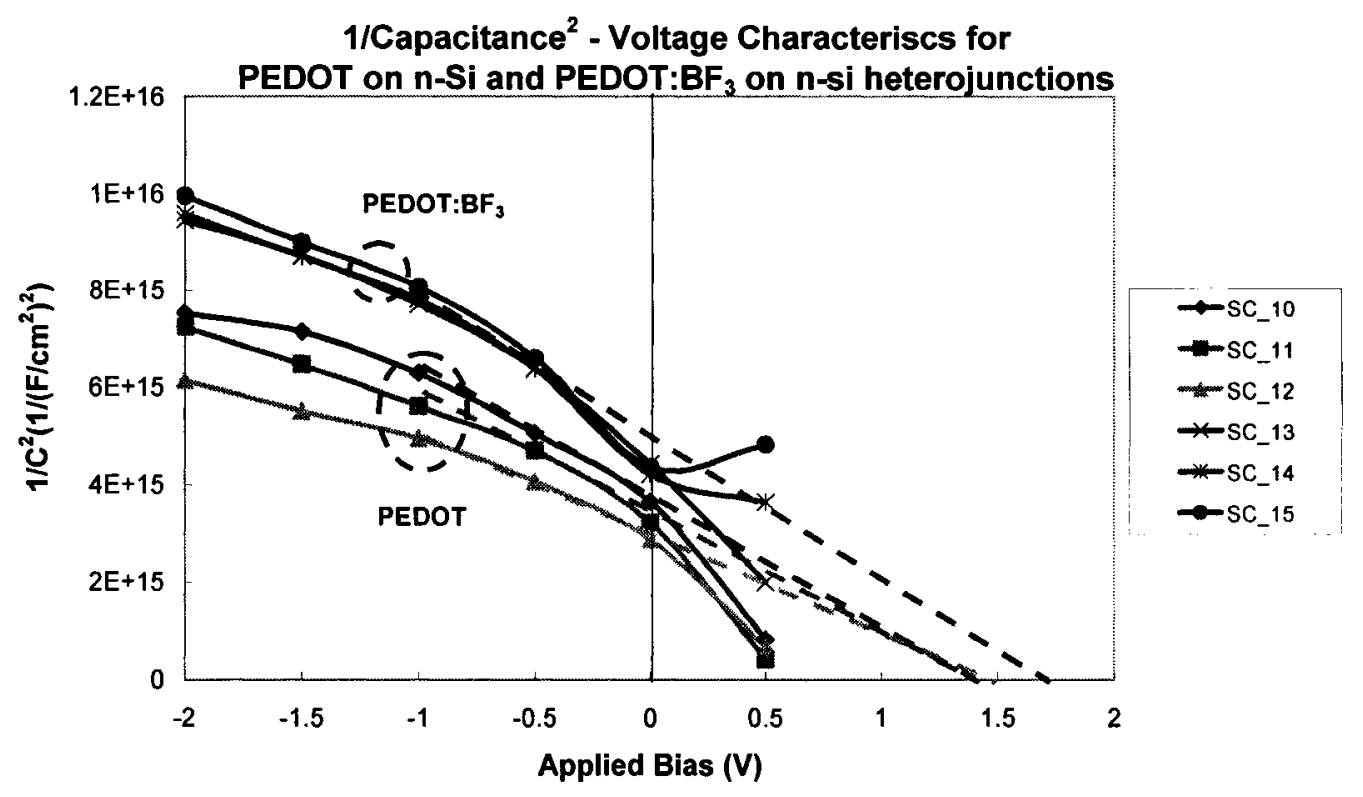

Figure 5.27: $1 / \mathrm{C}^{2}$ vs. voltage of PEDOT and PEDOT:BF 3 on $\mathrm{n}-\mathrm{Si}$ heterojunction devices.

From Figure 5.27 , the built-in voltage, $\mathrm{V}_{\mathrm{bi}}$ can be determined by extrapolating the curve to the point where $1 / C^{2}=0$. Assume that it is a one-sided junction, thus the $1 / C^{2}$ vs $V$ relationship can be simplified to Equation $(5-4)$ where the slope of the curve is inversely proportional to the doping concentration of the lightly doped region in the junction. The large reverse bias region or the so called deep region is considered for the calculation of these parameters as the curves are more linear due to the less pronounced effect of the interface states in the deep region. A summary of the $\mathrm{V}_{\mathrm{bi}}$ and doping concentration for the various devices is presented in Table 5.7. 
Table 5.7: Summary of the C-V characteristics for PEDOT and PEDOT:BF 3 on

n-Si heterojunctions with Au contact

\begin{tabular}{|c|c|c|c|c|}
\hline $\begin{array}{c}\text { Inorganic } \\
\text { Semiconductor }\end{array}$ & $\begin{array}{l}\text { Organic } \\
\text { Polymer }\end{array}$ & $\begin{array}{c}\text { Sample } \\
\#\end{array}$ & $V_{b i}(V)$ & $\mathbf{N}_{D}\left(\mathrm{~cm}^{-3}\right)$ \\
\hline \multirow{8}{*}{ n-type silicon } & \multirow{4}{*}{ PEDOT } & $S C_{-} 10$ & 1.4 & $4.9 \times 10^{15}$ \\
\hline & & $S C \_11$ & 1.4 & $6.6 \times 10^{15}$ \\
\hline & & $S C_{-} 12$ & 1.5 & $6.8 \times 10^{15}$ \\
\hline & & Average & 1.4 & $6.1 \times 10^{15}$ \\
\hline & \multirow{4}{*}{ PEDOT:BF 3} & $S C_{-} 13$ & 1.7 & $4.5 \times 10^{15}$ \\
\hline & & $S C \_14$ & 1.7 & $4.2 \times 10^{15}$ \\
\hline & & $S C_{-} 15$ & 1.7 & $4.1 \times 10^{15}$ \\
\hline & & Average & 1.7 & $4.3 \times 10^{15}$ \\
\hline
\end{tabular}

From Table 5.7, for PEDOT on n-Si heterojunction devices, the average built-in voltage, $\mathrm{V}_{\mathrm{bi}}$ is approximately $1.4 \mathrm{~V}$ while the doping concentration, $\mathrm{N}_{\mathrm{D}}$, of the lightly doped $n$-type silicon is roughly $6.1 \times 10^{15} \mathrm{~cm}^{-3}$. On the other hand, the average $V_{b i}$ for PEDOT:BF3 on $\mathrm{n}-\mathrm{Si}$ heterojunction devices is slightly higher, $1.7 \mathrm{~V}$ while the doping concentration is $4.3 \times 10^{15} \mathrm{~cm}^{-3}$. If the lightly doped $\mathrm{n}$-region becomes depleted at a high applied reverse-bias, it is possible to obtain the width of the n-region, W $(\mathrm{cm})$, from its capacitance, $\mathrm{C}\left(\mathrm{F} / \mathrm{cm}^{2}\right)$, using the following equation:

$$
W=\frac{\varepsilon_{S} \cdot A}{C}
$$

where $A$ is area of the active region $\left(\mathrm{cm}^{-2}\right)$ and $\varepsilon s$ is the substrate permittivity. With the doping concentration and the depletion layer width, it is possible to obtain the 
doping profile of the lightly doped region in the n-type silicon. Figure 5.28 shows the approximate doping profile for a PEDOT on $\mathrm{n}-\mathrm{Si}$ heterojunction device (SC_11) as an example.

Doping profile for PEDOT on n-Si heterojunction (SC_11)

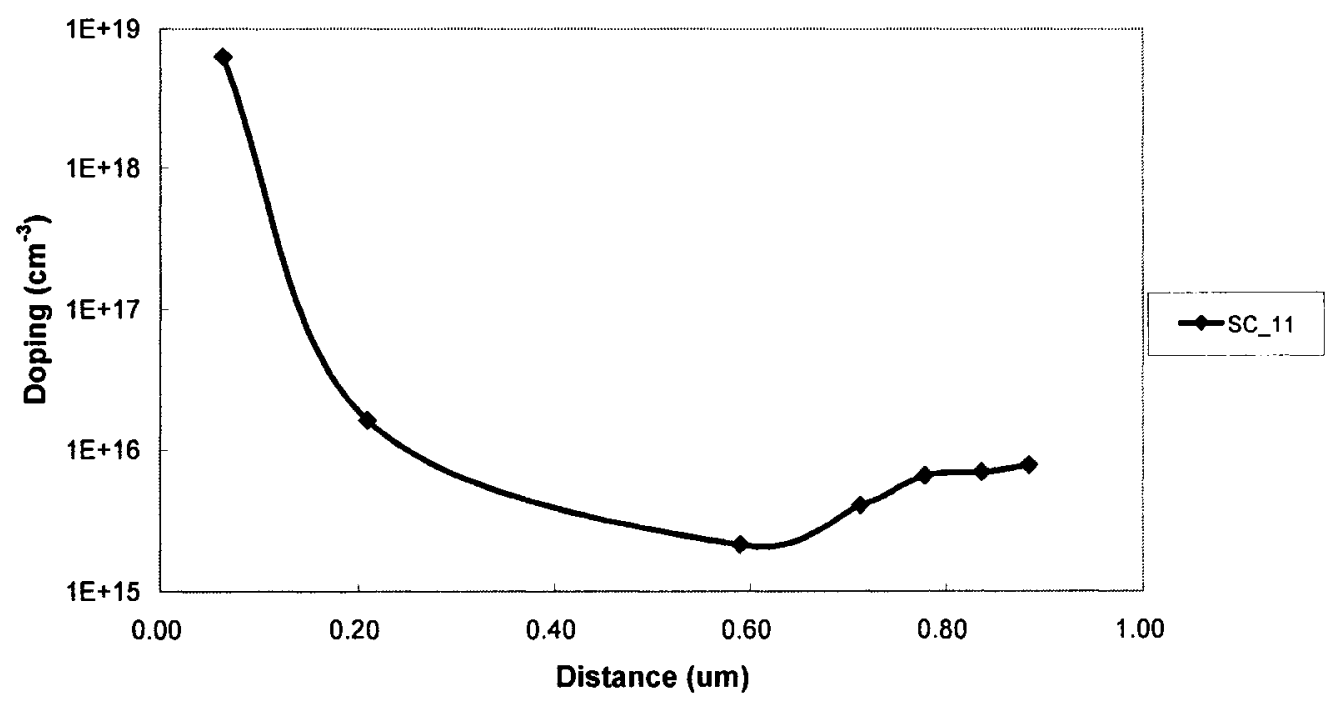

Figure 5.28: Doping profile for a PEDOT on n-Si heterojunction (SC_11) 


\subsection{Conclusions}

In this chapter, electrical characterization of organic on inorganic semiconductor heterojunctions has been performed. The inorganic semiconductor used is n-type silicon substrate while various organic polymers such as MEH-PPV POSS, RR-P3HT and PEDOT were incorporated as the organic counter-part. The experimental setups such as $\mathrm{I}-\mathrm{V}$ and $\mathrm{C}-\mathrm{V}$ measurements were briefly described followed by the results obtained for the different organic on inorganic semiconductor heterojunctions. A comparison of the parameters obtained from the I-V characteristics for different sets of heterojunctions were summarised in Table 5.7. Based on the experimental results, the following conclusions have been reached.

Table 5.7: Summary of the J-V characteristics for the organic on inorganic semiconductor heterojunctions

\begin{tabular}{|c|c|c|c|c|}
\hline Organic Polymer & $\begin{array}{c}M E H P P V \\
P O S S\end{array}$ & $R R$ P3HT & $P E D O T$ & $P E D O T: B F_{3}$ \\
\hline Anode Contact & $\boldsymbol{A l}$ & $\boldsymbol{A l}$ & $\boldsymbol{A u}$ & $\boldsymbol{A u}$ \\
\hline $\begin{array}{c}\text { Rectification Ratio } \\
(|\mathbf{1 V}|)\end{array}$ & $6.4 \times 10$ & $1.1 \times 10^{3}$ & $1.2 \times 10^{4}$ & $5.1 \times 10^{4}$ \\
\hline $\begin{array}{c}\text { Rectification Ratio } \\
(|\mathbf{2 V}|)\end{array}$ & $4.7 \times 10^{2}$ & $1.4 \times 10^{3}$ & $2.0 \times 10^{4}$ & $5.7 \times 10^{4}$ \\
\hline $\begin{array}{c}\text { Saturation Current } \\
\text { Density Jo (nA/cm } \mathbf{2})\end{array}$ & 23 & 22 & 15 & 9.7 \\
\hline $\begin{array}{c}\text { Leakage Current } \\
\text { Density ( } \mu \text { A/cm } \mathbf{2})\end{array}$ & 31 & 2.8 & 0.44 & 0.29 \\
\hline $\begin{array}{c}\text { Series Resistance } \\
(\Omega)\end{array}$ & $7.0 \times 10^{6}$ & $3.8 \times 10^{6}$ & $9.6 \times 10^{5}$ & $9.9 \times 10^{5}$ \\
\hline Ideality factor (n) & 1.7 & 2.0 & 2.9 & 2.3 \\
\hline
\end{tabular}


In general, almost all the organic on inorganic semiconductor heterojunctions regardless of the organic polymer or anode contact displayed a rectifying characteristic. From the various energy-band diagrams (Figure 5.12, 5.18 and 5.23) that have been constructed for the three sets of heterojunction devices in this work, it clearly shows that a potential barrier existed between the organic and inorganic semiconductor interface. During forward bias operation, electrons injected from the cathode into the $\mathrm{n}-\mathrm{Si}$ will encounter a potential barrier between the inorganic semiconductor and the organic polymer. Thus, the height of this potential barrier will affect the performance of the devices. Among the various heterojunctions, the PEDOT:BF $\mathrm{BF}_{3}$ on $\mathrm{n}-\mathrm{Si}$ heterojunctions with $\mathrm{Au}$ contact seem to have the best performance with high rectification ratio, small saturation current density and leakage current density. This could be accounted by the lowest potential barrier among the three heterojunctions and almost little or no energy level offset between the organic polymer and gold anode. However the ideality factor calculated was slightly larger compared to the MEH-PPV POSS or RR-P3HT on n-Si heterojunction devices. All the devices have fairly large series resistances in range of $\mathrm{M} \Omega$.

Device characteristics obtained with some of the polymer spin coated heterojunctions in this work appeared to be slightly inferior to those obtained by evaporation of PTCDA in Ref. 2.1 and 2.9. The PTCDA barrier diodes with In 
contacts have an ideality factor of 1.75 and extremely small series resistance. The differences could be accounted by the mobility of the material or quality of organic film and interfaces. Evaporation of the organic material usually produce a film with smaller grain size compared to that of polymer spin coated devices in this work. Thus, the interface quality might be better for evaporated PTCDA devices. The choice of contact materials might have an effect on the performance of the devices as well.

Investigation of the effect of anode contact metal on the heterojunction has revealed that if the energy level offset between the anode and the HOMO of the organic polymer is smaller, better performance will be achieved as concluded from the comparison between MEH-PPV POSS on $\mathrm{n}-\mathrm{Si}$ heterojunctions with $\mathrm{Al}$ and $\mathrm{Mg}-\mathrm{Ag}$ contact respectively. The work-function of $\mathrm{Al}$ is slightly higher than $\mathrm{Mg}-\mathrm{Ag}$ alloy and therefore a smaller energy level offset with MEH-PPV at their interface. In addition, as this energy barrier existed between the metal and organic polymer interface, the charge particles have a probability to tunnel through it.

Optical sensitivity measurements were performed with some the MEH-PPV POSS on n-Si heterojunctions and the I-V characteristics for the devices carried out in dark or under illuminated source showed little or no difference. It is not conclusive whether the organic on inorganic semiconductor heterojunctions are insensitive to light or there exists a high series resistance at the interface between the organic 
polymer and the anode which prevents the small photocurrent produced to be measured accurately. Therefore, it cannot be concluded whether the organic on inorganic semiconductor heterojunctions in this work are sensitive to illumination or not.

When the organic polymer PEDOT was doped with $\mathrm{BF}_{3}$, the heterojunctions displayed a better performance than those with undoped PEDOT. It is believed this is the first time that PEDOT:BF $\mathrm{B}_{3}$ on $\mathrm{n}-\mathrm{Si}$ heterojunctions characteristics have been reported. An increase in the magnitude of the output current density for PEDOT:BF 3 on $\mathrm{n}-\mathrm{Si}$ heterojunctions by a few times was observed. Furthermore, the built-in voltage, doping concentration of the lightly doped n-Si region or its doping profile have been derived from the capacitance - voltage characteristics.

In conclusion, the experimental results obtained provide a better understanding of the organic on inorganic semiconductor heterojunctions and possible enhancements that can be made to the heterojunctions to improve the overall efficiency. 


\subsection{References}

[5.1] Singh, Jaspirit (2001) "Semiconductor Devices: Basic Principles," John Wiley \& Sons, Inc. 187

[5.2] Neudeck, GW. (1989) "Modular series on solid state devices: The PN junction diode," $2^{\text {nd }}$ ed. Addison-Wesley Publishing Company.

[5.3] Tavakolian H. \& Sites, JR. (1985) Proceedings of $18^{\text {th }}$ IEEE PVSC, 1065

[5.4] McGuire, GE. and Strausser, YE, (1995) "Characterization in compound semiconductor processing," Butterworth-Heinemann 135

[5.5] Hadziioannou, G. \& Hutten, P.F. Van (2000) "Semiconducting Polymers Chemistry, Physics and Engineering", WILEY-VCH, U.S.

[5.6] Braun, D., Heeger, A.J. (1991) Appl. Phys. Lett., 58, 1982

[5.7] Hybrid inorganic-organic polymers (Ed: R.F. Stoney), (2000) in Polym. Prepr. American Chemical Society, Washington, DC, 41, 502-625

[5.8] Lee, ST. Hou, XY. Mason, MG. Tang, CW. (1998) Appl. Phys. Lett. 72, 13, 1593 [5.9] Jong Lae Kim et al. (2001) Syn. Met., 121, 1707

[5.10] Al-Ibrahim, M. Roth, H. Zholchavets, U. Gobsch, G. Sensfuss, S. (2005) Solar Energy Mats. \& Solar Cells, 85, 13-20

[5.11] Samuelsen EJ. and Mardalen J, (1997) "Handbook of Organic Conductive Molecules and Polymers," Vol 3, Chichester:Wiley 87-120

[5.12] Chen, TA.Wu, X. and Rieke, RD. (1995) J. Amer. Chem. Soc., 117, 233

[5.13] Bao, Z. Dodabalapur, A. and Lovinger, AJ. (1996) Appl. Phys. Lett., 69, 4108

[5.14] Gelinck, GH. et. al (2000) Applied Phy. Lett. Vol 77, 1487

[5.15] Thomas, CA. Zong, K. Schottland, P. and Reynolds, JR. (2000) Adv. Mater. Vol.

12,222

[5.16] Cui, XT. and Martin, DC. (2003) Sens Actuat. B: Chemical vol. 89, 92 
[5.17] Groenendaal, L. Jonas, F. Freitag, D. Pielartzik, H. \& Reynolds, JR. (2000) Adv.Mater. 12, 481

[5.18] Ho, PKH. Kim, J. Burroughes, JH. Becker, H. Li, SFY. Brown, TM. Cacialli, F. \& Friend, RH. (2000) Nature 404, 481 


\section{Conclusions and future work}

In the last decades, the growing interest in organic electronics has been a great force in promoting the research efforts made to improve the electrical and optical properties of the organic materials and their applications. Organic materials are known to have the key advantages of simple and low-temperature thin film processing through inexpensive techniques such as spin coating. In addition, the flexibility of organic chemistry enables the formation of organic molecules with useful luminescent and conducting properties. On the other hand, inorganic semiconductors offer the potential of a wide range of electrical properties, substantial mechanical hardness and thermal stability. Therefore, it is interesting to find out how these two materials can be incorporated together to form a functional electronic device. The objective of the thesis was thus to fabricate hybrid organic on inorganic semiconductor heterojunction devices and study their characteristics through simple electrical measurements as to have a better understanding of the operational principles behind these hybrid devices.

Organic on inorganic semiconductor heterojunction devices were fabricated using a sandwich structure on n-type silicon wafers. Electrical characterizations (I-V and $\mathrm{C}-\mathrm{V}$ ) of three sets of organic on inorganic semiconductor heterojunctions devices were carried out. The heterojunction devices include MEH-PPV POSS on n-Si, RR-P3HT on $\mathrm{n}-\mathrm{Si}$ and PEDOT on $\mathrm{n}-\mathrm{Si}$. All the three organic on inorganic 
semiconductor heterojunctions regardless of the conjugated polymer or anode contact displayed a rectifying characteristic. From the energy-band diagrams constructed for the three heterojunctions, there is a potential barrier existed at the organic and inorganic semiconductor interface. The potential barrier for the electrons during forward bias is determined by the energy difference between the LUMO of the conjugated polymer and the electron affinity of the n-Si. The performance of the devices appeared to be inversely proportional to the height of the barrier. MEH-PPV POSS on n-Si has the highest potential barrier followed by RR-P3HT on n-Si and lastly PEDOT on $\mathrm{n}-\mathrm{Si}$. From the experimental results, the rectification ratio at $|1 \mathrm{~V}|$ and $|2 \mathrm{~V}|$ varies from 64 to $5.1 \times 10^{4}$ and 470 to $5.7 \times 10^{4}$ respectively, with PEDOT:BF 3 on $\mathrm{n}$-Si having the highest rectification ratio. This is also the first time that PEDOT doped with $\mathrm{BF}_{3}$ on $\mathrm{n}-\mathrm{Si}$ heterojunction devices have been reported. The corresponding saturation current density and leakage current are $9.7 \mathrm{nA} / \mathrm{cm}^{2}$ and $0.29 \mu \mathrm{A} / \mathrm{cm}^{2}$ while the ideality factor is around 2.3 . The series resistance is fairly large for all the devices in the range of $M \Omega$.

Further investigation of the effect on anode contact metal on the heterojunction has shown that the smaller the energy level offset between the work-function of the metal and the HOMO of the conjugated polymer, the better performance the device will obtain. This is demonstrated by replacing the Al contact for MEH-PPV POSS on 
$\mathrm{n}-\mathrm{Si}$ heterojunction devices with a slightly lower work-function $\mathrm{Mg}$-Ag contact and comparing the difference in their I-V characteristics. The devices with $\mathrm{Mg}-\mathrm{Ag}$ contact appeared to have a slightly inferior performance. The same set of heterojunctions was also tested for optical sensitivity but the results showed little or no difference in dark or illuminated condition. It was not conclusive if the large series resistance existed across the conjugated polymer and the metal contact has hindered the measurement of the supposedly small photocurrent that should be observed. One of the major discoveries in this work is that doping PEDOT polymer with $\mathrm{BF}_{3}$ p-type dopant has improved the performance of the corresponding heterojunctions and an increase in the current magnitude by $2 \sim 3$ times was observed.

From the present work, the organic on inorganic semiconductor heterojunction device with the best performance was obtained by PEDOT:BF $\mathrm{B}_{3}$ on $\mathrm{n}-\mathrm{Si}$ with gold anode. These hybrid organic on inorganic heterojunctions still have not been studied extensively in this work; there are still many factors that can be explored in detail in the future. For example, annealing the devices at different temperatures may lead to a better formation at the organic-inorganic interface, resulting in an improvement to the device electrical characteristics. Secondly, it is evident that the series resistance greatly affects the device performance. Therefore, it is important to study the contact resistance between different metal anodes and the conjugated polymers and hopefully 
reduce this contact resistance so that it does not limit the performance of the devices. Thirdly, the inorganic semiconductor used in this work concentrated only on n-type silicon substrate. Thus it will be interesting to observe the effect when n-Si is replaced by other inorganic materials such as $\mathrm{p}-\mathrm{Si}, \mathrm{CdS}$ or GaAs while the p-type organic polymers may remain the same or be replaced by other potential n-type organics such as $\mathrm{C}_{60}$. Lastly, understanding the material characterisation of the organic polymers such as their film thickness, interface composition, geometrical characterisation and mobility in thin film will greatly improve the performance of the organic on inorganic semiconductor heterojunction devices. 